\title{
An Intrinsic Characterization of Five Points in a CAT(0) Space
}

https://doi.org/10.1515/agms-2020-0111

Received August 18, 2019; accepted May 20, 2020.

Abstract: Gromov (2001) and Sturm (2003) proved that any four points in a CAT(0) space satisfy a certain family of inequalities. We call those inequalities the $\nabla$-inequalities, following the notation used by Gromov. In this paper, we prove that a metric space $X$ containing at most five points admits an isometric embedding into a CAT(0) space if and only if any four points in $X$ satisfy the $\otimes$-inequalities. To prove this, we introduce a new family of necessary conditions for a metric space to admit an isometric embedding into a CAT(0) space by modifying and generalizing Gromov's cycle conditions. Furthermore, we prove that if a metric space satisfies all those necessary conditions, then it admits an isometric embedding into a CAT(0) space. This work presents a new approach to characterizing those metric spaces that admit an isometric embedding into a CAT(0) space.

Keywords: $\operatorname{CAT}(0)$ space; the $\otimes$-inequalities; the weighted quadruple inequalities; quadratic metric inequality; the $\operatorname{Cycl}_{k}(0)$ condition

MSC: Primary 53C23; Secondary 51F99

\section{Introduction}

Under the assumption that a metric space $X$ is geodesic, many simple conditions for $X$ that are equivalent to the condition that $X$ is a CAT(0) space have been known. For example, Berg and Nikolaev [3] proved that a metric space $\left(X, d_{X}\right)$ is $\operatorname{CAT}(0)$ if and only if $X$ is geodesic, and any $x, y, z, w \in X$ satisfy

$$
0 \leq d_{X}(x, y)^{2}+d_{X}(y, z)^{2}+d_{X}(z, w)^{2}+d_{X}(w, x)^{2}-d_{X}(x, z)^{2}-d_{X}(y, w)^{2}
$$

(see also Sato [16]). The inequality (1.1) was called the quadrilateral inequality in [3], and the roundness 2 inequality by Enflo [7] in connection with the geometry of Banach spaces.

On the other hand, when we characterize those metric spaces that admit an isometric embedding into a CAT(0) space, we have to omit such a non-intrinsic assumption that the ambient space is geodesic. Omitting the assumption that a metric space $X$ is geodesic changes the situation drastically. To see this, we recall the following family of inequalities.

Definition 1.1. We say that a metric space $\left(X, d_{X}\right)$ satisfies the $\otimes$-inequalities if for any $t, s \in[0,1]$ and any $x, y, z, w \in X$, we have

$$
\begin{aligned}
0 \leq(1-t)(1-s) d_{X}(x, y)^{2}+t(1-s) d_{X}(y, z)^{2}+t s d_{X}(z, w)^{2}+(1-t) s d_{X}(w, x)^{2} \\
-t(1-t) d_{X}(x, z)^{2}-s(1-s) d_{X}(y, w)^{2} .
\end{aligned}
$$

Gromov [10] and Sturm [17] introduced these inequalities independently, and proved that every CAT(0) space satisfies them. The name " $\otimes$-inequalities" is based on a notation used in [10], and was used in [12] and [18].

Tetsu Toyoda, Kogakuin University, Tokyo, Japan, E-mail: toyoda@cc.kogakuin.ac.jp 
In [17], they were called the weighted quadruple inequalities. When $s=t=1 / 2$, the $\nabla$-inequality becomes the quadrilateral inequality (1.1), and therefore a geodesic space satisfies the $\nabla$-inequalities if and only if it is CAT(0). The following example shows that there exists even a four-point metric space that satisfies the quadrilateral inequality (1.1) but does not admit an isometric embedding into any CAT(0) space.

Example 1.2. Let $X=\left\{x_{1}, x_{2}, x_{3}, x_{4}\right\}$. Define $d_{X}: X \times X \rightarrow[0, \infty)$ by

$$
\begin{aligned}
& d_{X}\left(x_{1}, x_{2}\right)=d_{X}\left(x_{2}, x_{3}\right)=d_{X}\left(x_{3}, x_{4}\right)=1, \\
& d_{X}\left(x_{4}, x_{1}\right)=d_{X}\left(x_{1}, x_{3}\right)=d_{X}\left(x_{2}, x_{4}\right)=\sqrt{3} .
\end{aligned}
$$

Then it is easily observed that $\left(X, d_{X}\right)$ is a metric space, and satisfies the quadrilateral inequality (1.1). However, $\left(X, d_{X}\right)$ does not satisfy the $\otimes$-inequality for $s=t=1 /(1+\sqrt{3})$, and therefore does not admit an isometric embedding into any $\operatorname{CAT}(0)$ space because every $\operatorname{CAT}(0)$ space satisfies the $\otimes$-inequalities.

To find a characterization of those metric spaces that admit an isometric embedding into a CAT(0) space is a longstanding open problem stated by Gromov in [10, §15] and [9, Section 1.19+] (see also [2, Section 1.4]). Every metric space containing at most three points admits an isometric embedding into a CAT(0) space because it admits an isometric embedding into the Euclidean plane. Gromov stated in [10, §7] that a four-point metric space admits an isometric embedding into a CAT(0) space if and only if it satisfies the $\nabla$-inequalities (see Theorem 1.7 below). In this paper, we find, for the first time, a characterization of those five-point metric spaces that admit an isometric embedding into a CAT(0) space. The following theorem is our main result.

Theorem 1.3. A metric space that contains at most five points admits an isometric embedding into a CAT(0) space if and only if it satisfies the $\bowtie$-inequalities.

Our proof of Theorem 1.3 also gives another proof of Gromov's characterization of those four-point metric spaces that admit an isometric embedding into a CAT(0) space whose detailed proof was omitted in [10].

\subsection{Gromov's cycle conditions and their generalizations}

To prove Theorem 1.3, we introduce new necessary conditions for a metric space to admit an isometric embedding into a CAT(0) space by slightly modifying and generalizing Gromov's cycle conditions defined in [10]. First we briefly recall some definitions and facts established mainly in [10]. In this paper, graphs are always assumed to be simple and undirected.

Definition 1.4 (Gromov [10]). Fix an integer $k \geq 4$. Let $G=(V, E)$ be the $k$-vertex cycle graph with vertex set $V$ and edge set $E$. A metric space $\left(X, d_{X}\right)$ is said to satisfy the $\operatorname{Cycl}_{k}(0)$ condition if for any map $f: V \rightarrow X$, there exists a map $g: V \rightarrow \mathbb{R}^{2}$ such that

$$
\begin{cases}\|g(u)-g(v)\| \leq d_{X}(f(u), f(v)), & \text { if }\{u, v\} \in E \\ \|g(u)-g(v)\| \geq d_{X}(f(u), f(v)), & \text { if }\{u, v\} \notin E\end{cases}
$$

for any $u, v \in V$.

Gromov [10] proved that every $\operatorname{CAT}(0)$ space satisfies the $\mathrm{Cycl}_{k}(0)$ condition for every integer $k \geq 4$. He also stated the following fact in $[10, \S 7]$.

Theorem 1.5 (Gromov [10]). A metric space satisfies the $\mathrm{Cycl}_{4}(0)$ condition if and only if it satisfies the $\square$ inequalities.

For a detailed proof of this theorem, see $[18, \S 7]$. Because a geodesic space satisfies the $\nabla$-inequalities if and only if it is $\operatorname{CAT}(0)$, it follows from Theorem 1.5 that a geodesic space satisfies the $\mathrm{Cycl}_{4}(0)$ condition if and 
only if it is $\operatorname{CAT}(0)$. This implies in particular that the $\mathrm{Cycl}_{4}(0)$ condition implies the $\mathrm{Cycl}_{k}(0)$ conditions for all integers $k \geq 4$ under the assumption that the metric space is geodesic. Recently, the present author [18] proved that this implication is true even without assuming that the metric space is geodesic.

Theorem 1.6 ([18]). If a metric space $X$ satisfies the $\mathrm{Cycl}_{4}(0)$ condition, or equivalently, if $X$ satisfies the $\square$ inequalities, then $X$ satisfies the $\operatorname{Cycl}_{k}(0)$ condition for every integer $k \geq 4$.

Moreover, it was also stated in $[10, \S 7]$ that any four-point metric space embeds isometrically into a threedimensional Riemannian space form of constant curvature at most 0 or a metric tree whenever it satisfies the $\mathrm{Cycl}_{4}(0)$ condition. Thus the following theorem holds.

Theorem 1.7 (Gromov [10]). A four-point metric space admits an isometric embedding into a CAT(0) space if and only if it satisfies the $\mathrm{Cycl}_{4}(0)$ condition.

Theorem 1.6 and Theorem 1.7 tell us that the $\mathrm{Cycl}_{4}(0)$ condition implies many necessary conditions for a metric space to admit an isometric embedding into a CAT(0) space. Therefore, it seems natural to ask whether the $\mathrm{Cycl}_{4}(0)$ condition (or the validity of the $\triangle$-inequalities) implies the isometric embeddability into a CAT(0) space or not. However, the answer of this question turned out to be false. Recently, Eskenazis, Mendel and Naor [8] proved that there exists a metric space that does not admit a coarse embedding into any CAT(0) space. On the other hand, it was proved in [12, Proposition 3.1] that for any $0<\alpha \leq 1 / 2$ and any metric space $\left(X, d_{X}\right)$, the metric space $\left(X, d_{X}^{\alpha}\right)$ satisfies the $\mathrm{Cycl}_{4}(0)$ condition. Therefore, if we choose a metric space $\left(Y, d_{Y}\right)$ that does not admit a coarse embedding into any $\operatorname{CAT}(0)$ space and a constant $0<\alpha \leq 1 / 2$, then the metric space $\left(Y, d_{Y}^{\alpha}\right)$ satisfies the $\mathrm{Cycl}_{4}(0)$ condition but does not admit a coarse embedding into any CAT(0) space because $\left(Y, d_{Y}^{\alpha}\right)$ is coarsely equivalent to $\left(Y, d_{Y}\right)$.

In this paper, to examine further to what extent the $\mathrm{Cycl}_{4}(0)$ condition implies necessary conditions for a metric space to admit an isometric embedding into a CAT(0) space, we define the following new conditions.

Definition 1.8. Let $G=(V, E)$ be a graph with vertex set $V$ and edge set $E$. A metric space $\left(X, d_{X}\right)$ is said to satisfy the $G(0)$ condition if for any map $f: V \rightarrow X$, there exist a $\operatorname{CAT}(0)$ space $\left(Y, d_{Y}\right)$ and a map $g: V \rightarrow Y$ such that

$$
\begin{cases}d_{Y}(g(u), g(v)) \leq d_{X}(f(u), f(v)), & \text { if }\{u, v\} \in E, \\ d_{Y}(g(u), g(v)) \geq d_{X}(f(u), f(v)), & \text { if }\{u, v\} \notin E\end{cases}
$$

for any $u, v \in V$.

Recently, Lebedeva, Petrunin and Zolotov [14] also introduced a similar condition. In the definition of their condition in [14, Section 8], a CAT(0) space $Y$ in Definition 1.8 is replaced with a Hilbert space. It is easily observed that every CAT(0) space satisfies the $G(0)$ condition for every graph $G$. Therefore, for every graph $G$, the $G(0)$ condition is a necessary condition for a metric space to admit an isometric embedding into a CAT(0) space. In Section 4, we will prove the following proposition, which states that the $G(0)$ conditions for all graphs $G$ form a necessary and sufficient condition for a metric space to admit an isometric embedding into a $\operatorname{CAT}(0)$ space.

Proposition 1.9. Fix a positive integer n. An n-point metric space admits an isometric embedding into a CAT(0) space if and only if it satisfies the $G(0)$ condition for every graph $G$ with $n$ vertices.

Clearly, for each integer $k \geq 4$, the $\operatorname{Cycl}_{k}(0)$ condition implies the $G(0)$ condition for the cycle graph $G$ with $k$ vertices. Therefore, it follows from Theorem 1.6 that the $\operatorname{Cycl}_{4}(0)$ condition (or the validity of the $\nabla$ inequalities) implies the $G(0)$ conditions for all cycle graphs $G$. In Sections 5, 6, 8 and 9, we will prove that the $\mathrm{Cycl}_{4}(0)$ condition also implies the $G(0)$ conditions for many finite graphs $G$ including all graphs containing at most five vertices. Together with Proposition 1.9, this proves Theorem 1.3, and also gives another proof of Theorem 1.7 whose detailed proof was omitted in [10]. 


\subsection{Quadratic metric inequalities that hold true in every CAT(0) space}

Homogeneous linear inequalities on the squares of distances among finite points like the $\nabla$-inequalities were called quadratic metric inequalities by Andoni, Naor, and Neiman [2]. In this paper, by slightly modifying their notation, we use the following notation to denote a quadratic metric inequality. For any positive integer $n$, we denote $[n]=\{1,2, \ldots, n\}$, and for any set $V$, we denote by $\left(\begin{array}{l}V \\ 2\end{array}\right)$ the set of all two-element subsets of $V$.

Definition 1.10. Fix a positive integer $n$. Let $E=\left(\begin{array}{c}{[n]} \\ 2\end{array}\right)$, and let $\left(a_{i j}\right)_{\{i, j\} \in E}$ be a family of real numbers indexed by $E$. A metric space $\left(X, d_{X}\right)$ is said to satisfy the $\left(a_{i j}\right)$-quadratic metric inequality if any points $x_{1}, \ldots, x_{n} \in X$ satisfy

$$
0 \leq \sum_{\{i, j\} \in E} a_{i j} d_{X}\left(x_{i}, x_{j}\right)^{2}
$$

The following theorem was proved in [2].

Theorem 1.11 (Andoni, Naor, and Neiman [2]). Let $n$ be a positive integer. An n-point metric space X admits an isometric embedding into a $\mathrm{CAT}(0)$ space if and only if $X$ satisfies the $\left(a_{i j}\right)$-quadratic metric inequality for every family $\left(a_{i j}\right)_{\{i, j\} \in E}$ of real numbers indexed by $E=\left(\begin{array}{c}{[n]} \\ 2\end{array}\right)$ such that every CAT(0) space satisfies the $\left(a_{i j}\right)$-quadratic metric inequality.

For the original statement of Theorem 1.11 in full generality, see [2, Proposition 3]. Theorem 1.11 tells us that characterizations of those metric spaces that admit an isometric embedding into a CAT(0) space follow from characterizations of those quadratic metric inequalities that hold true in every $\mathrm{CAT}(0)$ space. We will prove the following lemma in Section 4.

Lemma 1.12. Fix a positive integer $n$. Let $V=[n]$, and let $E=\left(\begin{array}{c}V \\ 2\end{array}\right)$. Suppose $A=\left(a_{i j}\right)_{\{i, j\} \in E}$ is a family of real numbers indexed by $E$ such that every CAT(0) space satisfies the $\left(a_{i j}\right)$-quadratic metric inequality. Let $E_{+}(A) \subseteq E$ be the set of all $\{i, j\} \in E$ with $a_{i j}>0$, and let $G_{A}=\left(V, E_{+}(A)\right)$ be the graph with vertex set $V$ and edge set $E_{+}(A)$. If a metric space satisfies the $G_{A}(0)$ condition, then it satisfies the $\left(a_{i j}\right)$-quadratic metric inequality.

We call the graph $G_{A}$ as in the statement of Lemma 1.12 the graph associated to the $\left(a_{i j}\right)$-quadratic metric inequality. Proposition 1.9 follows immediately from Lemma 1.12 and Theorem 1.11. It also follows from Lemma 1.12 that if every metric space satisfies the $G_{A}(0)$ condition for the graph $G_{A}$ associated to the $\left(a_{i j}\right)$-quadratic metric inequality, then the $\left(a_{i j}\right)$-quadratic metric inequality holds true in every metric space whenever it holds true in every CAT(0) space. In Section 5, we will prove that every metric space satisfies the $G(0)$ conditions for many graphs $G$ (including all trees for example).

\subsection{Some questions}

We pose the following questions.

Question 1.13. Find a graph $G$ such that there exists a metric space $X$ such that $X$ satisfies the $\operatorname{Cycl}_{4}(0)$ condition, but $X$ does not satisfy the $G(0)$ condition.

Question 1.14. Find a quadratic metric inequality J that satisfies the following two conditions:

(i) Every CAT(0) space satisfies J.

(ii) There exists a metric space that satisfies the $\otimes$-inequalities but that does not satisfy J.

Question 1.15. Find a characterization of those graphs $G$ such that every metric space satisfies the $G(0)$ condition. 


\subsection{Organization of the paper}

The paper is organized as follows. In Section 2, we recall some definitions and results from metric geometry. In Section 3, we recall and establish some properties of metric spaces that satisfy the $\nabla$-inequalities. In Section 4, we prove Lemma 1.12 and Proposition 1.9. In Section 5, we prove that the validity of the $\triangle$-inequalities implies the $G(0)$ condition for every graph $G$ containing at most four vertices. Combining this with Proposition 1.9, we obtain another proof of Theorem 1.7. In Section 5, we also specify several graphs $G$ such that every metric space satisfies the $G(0)$ condition. Combining this with Lemma 1.12, we obtain a criterion for a quadratic metric inequality to hold true in every metric space whenever it holds true in every CAT(0) space. In Section 6, we prove that the validity of the $\otimes$-inequalities implies the $G(0)$ condition for any graph $G$ with five vertices except two special graphs. In Section 7, we introduce certain concepts concerning the isometric embeddability of a four-point metric space into a Euclidean space. In Section 8 and Section 9, we prove that the validity of the $\triangle$-inequalities implies the $G(0)$ conditions for the remaining two graphs $G$ with five vertices by using the concepts introduced in Section 7. Together with Proposition 1.9, this completes the proof of Theorem 1.3.

\section{Preliminaries}

In this section, we set up some notations, and review some definitions and results in metric geometry. Throughout this paper, for every positive integer $n, \mathbb{R}^{n}$ is always equipped with the Euclidean metric. For distinct points $x, y \in \mathbb{R}^{n}$, we denote by $\overleftrightarrow{x y}$ the straight line through $x$ and $y$. For $x, y, z \in \mathbb{R}^{2}$ with $x \neq y$ and $y \neq z$, we denote by $\angle x y z \in[0, \pi]$ the interior angle measure at $y$ of the (possibly degenerate) triangle with vertices $x, y$ and $z$.

A geodesic in a metric space $X$ is an isometric embedding of an interval of the real line into $X$. For $x, y \in X$, we call the image of a geodesic $\gamma:\left[0, d_{X}(x, y)\right] \rightarrow X$ with $\gamma(0)=x$ and $\gamma\left(d_{X}(x, y)\right)=y$ a geodesic segment with endpoints $x$ and $y$. A metric space $X$ is called geodesic if for any $x, y \in X$, there exists a geodesic segment with endpoints $x$ and $y$.

Definition 2.1. A metric space $\left(X, d_{X}\right)$ is called a CAT(0) space if $X$ is geodesic, and any $x, y, z \in X$ and any geodesic $\gamma:\left[0, d_{X}(x, y)\right] \rightarrow X$ with $\gamma(0)=x$ and $\gamma\left(d_{X}(x, y)\right)=y$ satisfy

$$
d_{X}\left(z, \gamma\left(t d_{X}(x, y)\right)\right)^{2} \leq(1-t) d_{X}(x, z)^{2}+t d_{X}(y, z)^{2}-t(1-t) d_{X}(x, y)^{2}
$$

for any $t \in[0,1]$.

In $\mathbb{R}^{n}$, the inequality (2.1) always holds with equality. A subset $S$ of a geodesic space $X$ is called convex if any geodesic segment in $X$ with endpoints $x$ and $y$ is contained in $S$ whenever $x, y \in S$. Clearly, a convex subset of a CAT(0) space equipped with the induced metric is a CAT(0) space. A geodesic space $X$ is called uniquely geodesic if for any $x, y \in X$, a geodesic segment in $X$ with endpoints $x$ and $y$ is unique. It is easily observed that every CAT(0) space is uniquely geodesic. For any points $x$ and $y$ in a uniquely geodesic space, we denote the geodesic segment with endpoints $x$ and $y$ by $[x, y]$. We also denote the sets $[x, y] \backslash\{x, y\},[x, y] \backslash\{x\}$ and $[x, y] \backslash\{y\}$ by $(x, y),(x, y]$ and $[x, y)$, respectively. For a subset $S$ of a uniquely geodesic space $X$, the convex hull of $S$ is the intersection of all convex subsets of $X$ containing $S$, or equivalently, the minimal convex subset of $X$ that contains $S$. We denote the convex hull of $S$ by $\operatorname{conv}(S)$.

For a family of metric spaces $\left(X_{\alpha}, d_{\alpha}\right)_{\alpha \in A}$, we equip the disjoint union

$$
\coprod_{\alpha \in A} X_{\alpha}=\bigcup_{\alpha \in A} X_{\alpha} \times\{\alpha\}
$$

with the metric $d$ defined by

$$
d\left((x, \alpha),\left(x^{\prime}, \alpha^{\prime}\right)\right)= \begin{cases}d_{\alpha}\left(x, x^{\prime}\right) & \text { if } \alpha=\alpha^{\prime}, \\ \infty & \text { otherwise }\end{cases}
$$


We usually identify each set $X_{\alpha}$ with its image under the natural inclusion into $\bigsqcup_{\alpha \in A} X_{\alpha}$.

Suppose $\left(X, d_{X}\right)$ is a metric space with possibly infinite metrics, and $\sim$ is an equivalence relation on $X$ such that every equivalence class of $X$ by $\sim$ is closed. Let $\bar{X}=X / \sim$ be the set of all equivalence classes by $\sim$. For $\bar{x}, \bar{y} \in \bar{X}$, we define

$$
\bar{d}(\bar{x}, \bar{y})=\inf \sum_{i=1}^{k} d_{X}\left(x_{i}, y_{i}\right),
$$

where the infimum is taken over all sequences $x_{1}, y_{1}, x_{2}, y_{2}, \ldots, x_{k}, y_{k}$ in $X$ such that $x_{1} \in \bar{x}, y_{k} \in \bar{y}$, and $y_{i} \sim x_{i+1}$ for every $i \in \mathbb{Z} \cap[1, k-1]$. Then $\bar{d}$ becomes a metric on $\bar{X}$, which is called the quotient metric on $\bar{X}$.

Suppose that $\left(X_{1}, d_{1}\right)$ and $\left(X_{2}, d_{2}\right)$ are metric spaces, and that $Z_{1}$ and $Z_{2}$ are closed subsets of $X_{1}$ and $X_{2}$, respectively. Suppose further that $Z_{1}$ and $Z_{2}$ are isometric via an isometry $f: Z_{1} \rightarrow Z_{2}$. Define $\sim$ to be the equivalence relation on the disjoint union $X_{1} \sqcup X_{2}$ generated by the relations $z \sim f(z)$ for all $z \in Z_{1}$. Let $X_{0}=\left(X_{1} \sqcup X_{2}\right) / \sim$ be the set of all equivalence classes by the equivalence relation $\sim$, and let $d_{0}$ be the quotient metric on $X_{0}$. Then $\left(X_{0}, d_{0}\right)$ is the metric space called the gluing of $X_{1}$ and $X_{2}$ along the isometry $f$. We note that the natural inclusions of $X_{1}$ and $X_{2}$ into $X_{0}$ are both isometric embeddings. Assume in addition that $X_{1}$ and $X_{2}$ are complete locally compact CAT(0) spaces, and that $Z_{1}$ and $Z_{2}$ are convex subsets of $X_{1}$ and $X_{2}$, respectively. Then by Reshetnyak's gluing theorem, the gluing of $X_{1}$ and $X_{2}$ along $f$ is a CAT(0) space. For a proof of this fact, see [15] or [5, Theorem 9.1.21]. A more general statement is in [4, Chapter II.11, Theorem 11.1]. When two geodesic segments $[a, b] \subseteq X_{1}$ and $[c, d] \subseteq X_{2}$ are isometric, we mean by "the metric space obtained by gluing $X_{1}$ and $X_{2}$ by identifying $[a, b]$ with $[c, d]$ " the gluing of $X_{1}$ and $X_{2}$ along the isometry $f:[a, b] \rightarrow[c, d]$ with $f(a)=c$ and $f(b)=d$.

A large number of important examples of CAT(0) spaces arise as piecewise Euclidean metric simplicial complexes. For detailed expositions of piecewise Euclidean metric simplicial complexes, see [4, Chapter I.7]. For our purposes, it suffices to keep in mind the following simple example.

Example 2.2. Suppose $p_{1}, x_{1}, y_{1}, p_{2}, y_{2}, z_{2}, p_{3}, z_{3}, x_{3} \in \mathbb{R}^{2}$ are distinct points such that

$$
\left\|p_{1}-y_{1}\right\|=\left\|p_{2}-y_{2}\right\|, \quad\left\|p_{2}-z_{2}\right\|=\left\|p_{3}-z_{3}\right\|, \quad\left\|p_{3}-x_{3}\right\|=\left\|p_{1}-x_{1}\right\| .
$$

Equip the subsets

$$
T_{1}=\operatorname{conv}\left(\left\{p_{1}, x_{1}, y_{1}\right\}\right), \quad T_{2}=\operatorname{conv}\left(\left\{p_{2}, y_{2}, z_{2}\right\}\right), \quad T_{3}=\operatorname{conv}\left(\left\{p_{3}, z_{3}, x_{3}\right\}\right)
$$

of $\mathbb{R}^{2}$ with the induced metrics, and regard them as disjoint metric spaces. Suppose

$$
f:\left[p_{1}, y_{1}\right] \rightarrow\left[p_{2}, y_{2}\right], \quad g:\left[p_{2}, z_{2}\right] \rightarrow\left[p_{3}, z_{3}\right], \quad h:\left[p_{3}, x_{3}\right] \rightarrow\left[p_{1}, x_{1}\right]
$$

are the isometries such that

$$
f\left(p_{1}\right)=p_{2}, \quad f\left(y_{1}\right)=y_{2}, \quad g\left(p_{2}\right)=p_{3}, \quad g\left(z_{2}\right)=z_{3}, \quad h\left(p_{3}\right)=p_{1}, \quad h\left(x_{3}\right)=x_{1} .
$$

Let $T$ be the quotient of the disjoint union $T_{1} \sqcup T_{2} \sqcup T_{3}$ by the equivalence relation $\sim$ generated by the relations $a \sim f(a), b \sim g(b)$ and $c \sim h(c)$ for all $a \in\left[p_{1}, y_{1}\right], b \in\left[p_{2}, z_{2}\right]$ and $c \in\left[p_{3}, x_{3}\right]$, and let $d_{T}$ be the quotient metric on $T$. Then $\left(T, d_{T}\right)$ is a metric space, and we call it the piecewise Euclidean metric simplicial complex constructed from $T_{1}, T_{2}$ and $T_{3}$ by identifying $\left[p_{1}, y_{1}\right] \subseteq T_{1}$ with $\left[p_{2}, y_{2}\right] \subseteq T_{2},\left[p_{2}, z_{2}\right] \subseteq T_{2}$ with $\left[p_{3}, z_{3}\right] \subseteq T_{3}$, and $\left[p_{3}, x_{3}\right] \subseteq T_{3}$ with $\left[p_{1}, x_{1}\right] \subseteq T_{1}$. It follows from a general criterion [4, p.207, Lemma 5.6] that $T$ becomes a CAT(0) space if and only if

$$
2 \pi \leq \angle x_{1} p_{1} y_{1}+\angle y_{2} p_{2} z_{2}+\angle z_{3} p_{3} x_{3} .
$$

We claim that it is easily observed that the above criterion holds true even if $T_{1}, T_{2}$ or $T_{3}$ is degenerate, or equivalently, even if some of the angles in the right-hand side of (2.2) take values in $\{0, \pi\}$. It is also easily observed that under the condition (2.2), the natural inclusions of $T_{1}, T_{2}$ and $T_{3}$ into $T$ are all isometric embeddings although a simplex in a metric simplicial complex is generally not embedded isometrically into the complex. 
Let $\left(X, d_{X}\right)$ be a metric space, and let $x, y, z \in X$ be points with $x \neq y$ and $y \neq z$. Then there exist $\tilde{x}, \tilde{y}, \tilde{z} \in \mathbb{R}^{2}$ such that

$$
\|\tilde{x}-\tilde{y}\|=d_{X}(x, y), \quad\|\tilde{y}-\tilde{z}\|=d_{X}(y, z), \quad\|\tilde{z}-\tilde{x}\|=d_{X}(z, x) .
$$

We define the comparison angle measure $\tilde{Z} x y z$ to be $\angle \tilde{x} \tilde{y} \tilde{z} \in[0, \pi]$. Clearly the comparison angle measure $\tilde{Z} x y z$ does not depend on the choice of $\tilde{x}, \tilde{y}, \tilde{z} \in \mathbb{R}^{2}$.

Definition 2.3. A geodesic space $X$ is said to have nonnegative Alexandrov curvature if, for any $p \in X$, there exists a neighborhood $U \subseteq X$ of $p$ such that any distinct four points $x, y, z, w \in U$ satisfy

$$
\tilde{\angle} y x z+\tilde{Z} z x w+\tilde{Z} w x y \leq 2 \pi .
$$

There are many equivalent definitions of metric spaces with nonnegative Alexandrov curvature. We refer [5] and [6] for detailed expositions of metric spaces with nonnegative Alexandrov curvature. For our purpose, it suffices to keep in mind the following two examples.

Example 2.4. Let $S$ be the boundary of a convex bounded subset of $\mathbb{R}^{3}$. Let $d_{S}$ be the induced length metric on $S$. In other words, for any $x, y \in S, d_{S}(x, y)$ coincides with the infimum of the lengths of all paths $\gamma:[a, b] \rightarrow S$ such that $\gamma(a)=x$ and $\gamma(b)=y$. It is known that $\left(S, d_{S}\right)$ has nonnegative Alexandrov curvature.

Example 2.5. Suppose $x, y$ and $z$ are points in $\mathbb{R}^{2}$ that are not collinear. Let $T_{1}$ and $T_{2}$ be two isometric copies of $\operatorname{conv}(\{x, y, z\})$. We denote the points in $T_{1}$ corresponding to $x, y$ and $z$ by $x_{1}, y_{1}$ and $z_{1}$, respectively, and the points in $T_{2}$ corresponding to $x, y$ and $z$ by $x_{2}, y_{2}$ and $z_{2}$, respectively. Suppose

$$
f:\left[x_{1}, y_{1}\right] \rightarrow\left[x_{2}, y_{2}\right], \quad g:\left[y_{1}, z_{1}\right] \rightarrow\left[y_{2}, z_{2}\right], \quad h:\left[z_{1}, x_{1}\right] \rightarrow\left[z_{2}, x_{2}\right]
$$

are the isometries such that

$$
f\left(x_{1}\right)=x_{2}, \quad f\left(y_{1}\right)=y_{2}, \quad g\left(y_{1}\right)=y_{2}, \quad g\left(z_{1}\right)=z_{2}, \quad h\left(z_{1}\right)=z_{2}, \quad h\left(x_{1}\right)=x_{2} .
$$

Let $T_{0}$ be the quotient of the disjoint union $T_{1} \sqcup T_{2}$ by the equivalence relation $\sim$ generated by the relations $a \sim f(a), b \sim g(b)$ and $c \sim h(c)$ for all $a \in\left[x_{1}, y_{1}\right], b \in\left[y_{1}, z_{1}\right]$ and $c \in\left[z_{1}, x_{1}\right]$, and let $d_{T_{0}}$ be the quotient metric on $T_{0}$. It is known that $\left(T_{0}, d_{T_{0}}\right)$ is a complete geodesic space with nonnegative Alexandrov curvature. Clearly the natural inclusions of $T_{1}$ and $T_{2}$ into $T_{0}$ are both isometric embeddings. We call the metric space $T_{0}$ defined above the piecewise Euclidean simplicial complex obtained by gluing $T_{1}$ and $T_{2}$ along their boundaries.

In [13], Lang and Schroeder generalized the classical Kirszbraun's extension theorem (see also [1]). The following is a part of their result, which we will use in Section 9. For the original statement in full generality, see $[13$, Theorem A].

Theorem 2.6 (Lang and Schroeder [13]). Suppose that $X$ is a complete geodesic space with nonnegative Alexandrov curvature and $Y$ is a complete CAT(0) space. Suppose that $S$ is a subset of $X$ and $f: S \rightarrow Y$ is a 1-Lipschitz map. Then there exists a 1-Lipschitz map $\tilde{f}: X \rightarrow Y$ such that $\tilde{f}(x)=f(x)$ for any $x \in S$.

Fix a positive integer $n$. Let $E_{n}=\left(\begin{array}{c}{[n]} \\ 2\end{array}\right)$ be the set of all two-element subsets of $[n]=\{1, \ldots, n\}$. Define $\mathcal{C}_{n}$ to be

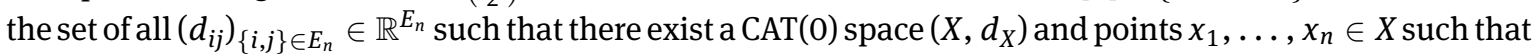
$d_{i j}=d_{X}\left(x_{i}, x_{j}\right)^{2}$ for every $\{i, j\} \in E_{n}$. Then $\mathcal{C}_{n}$ is a closed convex cone in $\mathbb{R}^{E_{n}}$. This follows immediately from the fact that the CAT(0) property is closed under taking Pythagorean product, taking dilation by a positive constant, and taking ultraproduct (see [17, Lemma 3.9] and [11, Section 2.4]). For completeness, we recall Andoni, Naor, and Neiman's proof of Theorem 1.11.

Proof of Theorem 1.11. Fix a positive integer $n$. The case in which $n=1$ is trivial, so we assume that $n \geq 2$. If an $n$-point metric space $X$ embeds isometrically into a CAT(0) space, then $X$ clearly satisfies every quadratic metric inequality that holds true in every $\operatorname{CAT}(0)$ space. We prove the converse direction by contrapositive. Assume that an $n$-point metric space $X=\left\{x_{1}, \ldots, x_{n}\right\}$ does not embed isometrically into a CAT(0) space. Then 
because $\mathcal{C}_{n} \subseteq \mathbb{R}^{E_{n}}$ is a closed convex cone, and $\left(d_{X}\left(x_{i}, x_{j}\right)^{2}\right)_{\{i, j\} \in E_{n}} \notin \mathcal{C}_{n}$, the separation theorem implies that there exists $\left(h_{i j}\right)_{\{i, j\} \in E_{n}} \in \mathbb{R}^{E_{n}}$ such that

$$
\inf _{\left(d_{i j}\right) \in \mathfrak{C}_{n}} \sum_{\{i, j\} \in E_{n}} h_{i j} d_{i j} \geq 0, \quad \sum_{\{i, j\} \in E_{n}} h_{i j} d_{X}\left(x_{i}, x_{j}\right)^{2}<0 .
$$

The first inequality in (2.3) means that the $\left(h_{i j}\right)$-quadratic metric inequality holds true in every CAT(0) space, and the second inequality means that $X$ does not satisfy the $\left(h_{i j}\right)$-quadratic metric inequality, which completes the proof.

\section{Comparison Quadrangles in the Euclidean plane}

In this section, we recall and establish some properties of metric spaces that satisfy the $\nabla$-inequalities. First, we recall the following fact, which was established by Sturm when he proved in [17, Theorem 4.9] that a geodesic space is $\operatorname{CAT}(0)$ whenever it satisfies the $\otimes$-inequalities.

Proposition 3.1. Let $\left(X, d_{X}\right)$ be a metric space that satisfies the $\otimes$-inequalities. Suppose $x, y, z \in X$ are points such that $x \neq z$, and

$$
d_{X}(x, z)=d_{X}(x, y)+d_{X}(y, z) .
$$

Set $t=d_{X}(x, y) / d_{X}(x, z)$. Then we have

$$
d_{X}(y, w)^{2} \leq(1-t) d_{X}(x, w)^{2}+t d_{X}(z, w)^{2}-t(1-t) d_{X}(x, z)^{2} .
$$

for any $w \in X$.

For the proof of Proposition 3.1, see [18, Proposition 7.1]. The following two lemmas will be used throughout this paper.

Lemma 3.2. Let $\left(X, d_{X}\right)$ be a metric space that satisfies the $\otimes$-inequalities. Suppose $x, y, z, w \in X$ and $x^{\prime}, y^{\prime}, z^{\prime}, w^{\prime} \in \mathbb{R}^{2}$ are points such that

$$
\begin{aligned}
& d_{X}(x, y) \leq\left\|x^{\prime}-y^{\prime}\right\|, \quad d_{X}(y, z) \leq\left\|y^{\prime}-z^{\prime}\right\|, \quad d_{X}(z, w) \leq\left\|z^{\prime}-w^{\prime}\right\|, \\
& d_{X}(w, x) \leq\left\|w^{\prime}-x^{\prime}\right\|, \quad\left\|x^{\prime}-z^{\prime}\right\| \leq d_{X}(x, z),
\end{aligned}
$$

and $\left[x^{\prime}, z^{\prime}\right] \cap\left[y^{\prime}, w^{\prime}\right] \neq \emptyset$. Then $d_{X}(y, w) \leq\left\|y^{\prime}-w^{\prime}\right\|$.

For the proof of Lemma 3.2, see [18, Corollary 5.2, Lemma 7.2].

Lemma 3.3. Let $\left(X, d_{X}\right)$ be a metric space that satisfies the $\nabla$-inequalities, and let $\left(Y, d_{Y}\right)$ be a metric space. Suppose $x, y, z, w \in X$ and $x^{\prime}, y^{\prime}, z^{\prime}, w^{\prime} \in Y$ are points such that

$$
\begin{aligned}
& d_{X}(x, y) \leq d_{Y}\left(x^{\prime}, y^{\prime}\right), \quad d_{X}(y, z) \leq d_{Y}\left(y^{\prime}, z^{\prime}\right), \quad d_{X}(z, w) \leq d_{Y}\left(z^{\prime}, w^{\prime}\right), \\
& d_{X}(w, x) \leq d_{Y}\left(w^{\prime}, x^{\prime}\right), \quad d_{Y}\left(x^{\prime}, z^{\prime}\right) \leq d_{X}(x, z) .
\end{aligned}
$$

Assume that there exist subsets $S$ and $T$ of $Y$ that satisfy the following conditions:

(1) $S$ and $T$ are isometric to convex subsets of Euclidean spaces.

(2) $\left\{x^{\prime}, y^{\prime}, z^{\prime}\right\} \subseteq S$ and $\left\{x^{\prime}, w^{\prime}, z^{\prime}\right\} \subseteq T$.

(3) There exists a geodesic segment $\Gamma_{1}$ in $Y$ with endpoints $x^{\prime}$ and $z^{\prime}$ such that $\Gamma_{1} \subseteq S \cap T$.

(4) There exists a point $p \in \Gamma_{1}$ such that $d_{Y}\left(y^{\prime}, w^{\prime}\right)=d_{Y}\left(y^{\prime}, p\right)+d_{Y}\left(p, w^{\prime}\right)$.

Then $d_{X}(y, w) \leq d_{Y}\left(y^{\prime}, w^{\prime}\right)$.

For the proof of Lemma 3.3, see [18, Corollary 5.3, Lemma 7.2]. 
Remark 3.4. Clearly we may replace the condition (4) in the statement of Lemma 3.3 with the following condition:

(4') There exists a geodesic segment $\Gamma_{2}$ in $Y$ with endpoints $y^{\prime}$ and $w^{\prime}$ such that $\Gamma_{1} \cap \Gamma_{2} \neq \emptyset$.

We will also use the following lemma.

Lemma 3.5. Let $\left(X, d_{X}\right)$ be a metric space that satisfies the $\otimes$-inequalities. Suppose $x, y, z, w \in X$ and $x^{\prime}, y^{\prime}, z^{\prime}, w^{\prime} \in \mathbb{R}^{2}$ are points with $z \neq w$ such that

$$
\begin{aligned}
& d_{X}(y, z) \leq\left\|y^{\prime}-z^{\prime}\right\|, \quad d_{X}(z, w) \leq\left\|z^{\prime}-w^{\prime}\right\|, \quad d_{X}(w, x) \leq\left\|w^{\prime}-x^{\prime}\right\|, \\
& \left\|x^{\prime}-z^{\prime}\right\| \leq d_{X}(x, z), \quad\left\|y^{\prime}-w^{\prime}\right\| \leq d_{X}(y, w),
\end{aligned}
$$

and $\left[x^{\prime}, z^{\prime}\right] \cap\left[y^{\prime}, w^{\prime}\right] \neq \emptyset$. Then $\left\|x^{\prime}-y^{\prime}\right\| \leq d_{X}(x, y)$.

Proof. We consider three cases.

CASE 1: $\left[x^{\prime}, z^{\prime}\right) \cap\left[y^{\prime}, w^{\prime}\right) \neq \emptyset$. In this case, there exist $s \in[0,1)$ and $t \in[0,1)$ such that

$$
(1-t) x^{\prime}+t z^{\prime}=(1-s) y^{\prime}+s w^{\prime} .
$$

It follows from this equality and the hypotheses of the lemma that

$$
\begin{aligned}
0= & \left\|\left((1-t) x^{\prime}+t z^{\prime}\right)-\left((1-s) y^{\prime}+s w^{\prime}\right)\right\|^{2} \\
= & (1-t)(1-s)\left\|x^{\prime}-y^{\prime}\right\|^{2}+t(1-s)\left\|y^{\prime}-z^{\prime}\right\|^{2}+t s\left\|z^{\prime}-w^{\prime}\right\|^{2}+(1-t) s\left\|w^{\prime}-x^{\prime}\right\|^{2} \\
& -t(1-t)\left\|x^{\prime}-z^{\prime}\right\|^{2}-s(1-s)\left\|y^{\prime}-w^{\prime}\right\|^{2} \\
\geq & (1-t)(1-s)\left\|x^{\prime}-y^{\prime}\right\|^{2}+t(1-s) d_{X}(y, z)^{2}+t s d_{X}(z, w)^{2}+(1-t) s d_{X}(w, x)^{2} \\
& -t(1-t) d_{X}(x, z)^{2}-s(1-s) d_{X}(y, w)^{2} .
\end{aligned}
$$

On the other hand,

$$
\begin{aligned}
0 & \leq(1-t)(1-s) d_{X}(x, y)^{2}+t(1-s) d_{X}(y, z)^{2}+t s d_{X}(z, w)^{2}+(1-t) s d_{X}(w, x)^{2} \\
& -t(1-t) d_{X}(x, z)^{2}-s(1-s) d_{X}(y, w)^{2}
\end{aligned}
$$

because $X$ satisfies the $\otimes$-inequalities. Comparing these yields

$$
\left\|x^{\prime}-y^{\prime}\right\| \leq d_{X}(x, y) .
$$

CASE 2: $\left[x^{\prime}, z^{\prime}\right) \cap\left[y^{\prime}, w^{\prime}\right)=\emptyset, x^{\prime} \neq z^{\prime}$ and $y^{\prime} \neq w^{\prime}$. In this case, $z^{\prime} \in\left[y^{\prime}, w^{\prime}\right]$ or $w^{\prime} \in\left[x^{\prime}, z^{\prime}\right]$ because $\left[x^{\prime}, z^{\prime}\right] \cap\left[y^{\prime}, w^{\prime}\right] \neq \emptyset$ by hypothesis. We assume without loss of generality that $z^{\prime} \in\left[y^{\prime}, w^{\prime}\right]$. Then

$$
d_{X}(y, w) \leq d_{X}(y, z)+d_{X}(z, w) \leq\left\|y^{\prime}-z^{\prime}\right\|+\left\|z^{\prime}-w^{\prime}\right\|=\left\|y^{\prime}-w^{\prime}\right\| \leq d_{X}(y, w),
$$

which implies that

$$
d_{X}(y, w)=d_{X}(y, z)+d_{X}(z, w)=\left\|y^{\prime}-z^{\prime}\right\|+\left\|z^{\prime}-w^{\prime}\right\|=\left\|y^{\prime}-w^{\prime}\right\| .
$$

The second equality in (3.1) implies that

$$
d_{X}(y, z)=\left\|y^{\prime}-z^{\prime}\right\|, \quad d_{X}(z, w)=\left\|z^{\prime}-w^{\prime}\right\| .
$$

Hence we can write

$$
z^{\prime}=(1-c) y^{\prime}+c w^{\prime}
$$

where

$$
c=\frac{\left\|y^{\prime}-z^{\prime}\right\|}{\left\|y^{\prime}-w^{\prime}\right\|}=\frac{d_{X}(y, z)}{d_{X}(y, w)}
$$


Because $0<d_{X}(z, w) \leq\left\|z^{\prime}-w^{\prime}\right\|$ by hypothesis, $z^{\prime} \neq w^{\prime}$ and $c \in[0,1)$. We have

$$
\begin{aligned}
d_{X}(x, z)^{2} & \geq\left\|x^{\prime}-z^{\prime}\right\|^{2} \\
& =\left\|x^{\prime}-(1-c) y^{\prime}-c w^{\prime}\right\|^{2} \\
& =(1-c)\left\|x^{\prime}-y^{\prime}\right\|^{2}+c\left\|x^{\prime}-w^{\prime}\right\|^{2}-c(1-c)\left\|y^{\prime}-w^{\prime}\right\|^{2} \\
& \geq(1-c)\left\|x^{\prime}-y^{\prime}\right\|^{2}+c d_{X}(x, w)^{2}-c(1-c) d_{X}(y, w)^{2} .
\end{aligned}
$$

On the other hand, (3.1), (3.2) and Proposition 3.1 imply that

$$
d_{X}(x, z)^{2} \leq(1-c) d_{X}(x, y)^{2}+c d_{X}(x, w)^{2}-c(1-c) d_{X}(y, w)^{2} .
$$

Comparing these yields

$$
\left\|x^{\prime}-y^{\prime}\right\| \leq d_{X}(x, y) .
$$

CASE 3: $x^{\prime}=z^{\prime}$ or $y^{\prime}=w^{\prime}$. In this case, we may assume without loss of generality that $x^{\prime}=z^{\prime}$. Then $x^{\prime} \in\left[y^{\prime}, w^{\prime}\right]$ because $\left[x^{\prime}, z^{\prime}\right] \cap\left[y^{\prime}, w^{\prime}\right] \neq \emptyset$. Therefore,

$$
\left\|x^{\prime}-y^{\prime}\right\|=\left\|y^{\prime}-w^{\prime}\right\|-\left\|w^{\prime}-x^{\prime}\right\| \leq d_{X}(y, w)-d_{X}(w, x) \leq d_{X}(x, y) .
$$

The above three cases exhaust all possibilities.

Remark 3.6. If we omit the condition that $z \neq w$ from the hypothesis of Lemma 3.5, then the statement becomes false. For example, suppose $\theta$ and $\theta^{\prime}$ are real numbers such that $0 \leq \theta<\theta^{\prime} \leq \pi$, and define points $x, y, z, w, x^{\prime}, y^{\prime}, z^{\prime}, w^{\prime} \in \mathbb{R}^{2}$ by

$$
\begin{aligned}
& x=(\cos \theta, \sin \theta), \quad y=(1,0), \quad z=w=(0,0), \\
& x^{\prime}=\left(\cos \theta^{\prime}, \sin \theta^{\prime}\right), \quad y^{\prime}=(1,0), \quad z^{\prime}=w^{\prime}=(0,0) .
\end{aligned}
$$

Then

$$
\begin{aligned}
& \left\|y^{\prime}-z^{\prime}\right\|=\|y-z\|, \quad\left\|z^{\prime}-w^{\prime}\right\|=\|z-w\|, \quad\left\|w^{\prime}-x^{\prime}\right\|=\|w-x\|, \\
& \left\|x^{\prime}-z^{\prime}\right\|=\|x-z\|, \quad\left\|y^{\prime}-w^{\prime}\right\|=\|y-w\|, \quad\left[x^{\prime}, z^{\prime}\right] \cap\left[y^{\prime}, w^{\prime}\right] \neq \emptyset .
\end{aligned}
$$

However,

$$
\|x-y\|<\left\|x^{\prime}-y^{\prime}\right\|
$$

\section{A criterion for isometric embeddability into a CAT(0) space}

In this section, we prove Lemma 1.12 and Proposition 1.9. We first prove Lemma 1.12.

Proof of Lemma 1.12. Let $\left(X, d_{X}\right)$ be a metric space that satisfies the $G_{A}(0)$ condition, and let $x_{1}, \ldots, x_{n} \in X$. Then there exist a $\operatorname{CAT}(0)$ space $\left(Y, d_{Y}\right)$ and points $y_{1}, \ldots, y_{n} \in Y$ such that

$$
\begin{cases}d_{Y}\left(y_{i}, y_{j}\right) \leq d_{X}\left(x_{i}, x_{j}\right), & \text { if }\{i, j\} \in E_{+}(A), \\ d_{Y}\left(y_{i}, y_{j}\right) \geq d_{X}\left(x_{i}, x_{j}\right), & \text { if }\{i, j\} \notin E_{+}(A)\end{cases}
$$

for any $i, j \in V$. Because $Y$ satisfies the $\left(a_{i j}\right)$-quadratic metric inequality by hypothesis, we have

$$
\begin{aligned}
0 & \leq \sum_{\{i, j\} \in E} a_{i j} d_{Y}\left(y_{i}, y_{j}\right)^{2} \\
& =\sum_{\{i, j\} \in E_{+}(A)}\left|a_{i j}\right| d_{Y}\left(y_{i}, y_{j}\right)^{2}-\sum_{\{i, j\} \in E \backslash E_{+}(A)}\left|a_{i j}\right| d_{Y}\left(y_{i}, y_{j}\right)^{2} \\
& \leq \sum_{\{i, j\} \in E_{+}(A)}\left|a_{i j}\right| d_{X}\left(x_{i}, x_{j}\right)^{2}-\sum_{\{i, j\} \in E \backslash E_{+}(A)}\left|a_{i j}\right| d_{X}\left(x_{i}, x_{j}\right)^{2} \\
& =\sum_{\{i, j\} \in E} a_{i j} d_{X}\left(x_{i}, x_{j}\right)^{2},
\end{aligned}
$$

which proves that $X$ satisfies the $\left(a_{i j}\right)$-quadratic metric inequality. 
Proposition 1.9 follows from Lemma 1.12 and Theorem 1.11.

Proof of Proposition 1.9. Let $\left(X, d_{X}\right)$ be an $n$-point metric space. If $X$ admits an isometric embedding into a CAT(0) space, then $X$ satisfies the $G(0)$ condition for every graph $G$ with $n$ vertices because every CAT(0) space satisfies the $G(0)$ condition. Conversely, suppose that $X$ satisfies the $G(0)$ condition for every graph $G$ with $n$ vertices. Let $V=[n]$, and let $E=\left(\begin{array}{c}V \\ 2\end{array}\right)$. Fix a family $A=\left(a_{i j}\right)_{\{i, j\} \in E}$ of real numbers indexed by $E$ such that every $\operatorname{CAT}(0)$ space satisfies the $\left(a_{i j}\right)$-quadratic metric inequality. Let $E_{+}(A) \subseteq E$ be the set of all $\{i, j\} \in E$ such that $a_{i j}>0$, and let $G_{A}=\left(V, E_{+}(A)\right)$ be the graph with vertex set $V$ and edge set $E_{+}(A)$. Then $X$ satisfies the $G_{A}(0)$ condition, and therefore $X$ satisfies the $\left(a_{i j}\right)$-quadratic metric inequality by Lemma 1.12 . Thus it follows from Theorem 1.11 that $X$ admits an isometric embedding into a CAT(0) space.

\section{Four points in a CAT(0) space}

In this section, we prove that if a metric space satisfies the $\otimes$-inequalities, then it satisfies the $G(0)$ condition for every graph $G$ with four vertices. Together with Proposition 1.9, this gives another proof of Theorem 1.7. We first observe that there are many graphs $G$ such that every metric space satisfies the $G(0)$ condition. As we declared before, graphs are always assumed to be simple and undirected.

Proposition 5.1. Let $G=(V, E)$ be a finite graph. Assume that there exists a vertex $v_{0} \in V$ such that $\{u, v\} \in E$ for any $u, v \in V \backslash\left\{v_{0}\right\}$ with $u \neq v$. Then every metric space satisfies the $G(0)$ condition.

Proof. Let $\left(X, d_{X}\right)$ be a metric space. For each map $f: V \rightarrow X$, define a map $g: V \rightarrow \mathbb{R}$ by $g(v)=$ $d_{X}\left(f\left(v_{0}\right), f(v)\right)$. Then

$$
|g(u)-g(v)|=\left|d_{X}\left(f\left(v_{0}\right), f(u)\right)-d_{X}\left(f\left(v_{0}\right), f(v)\right)\right| \leq d_{X}(f(u), f(v))
$$

for any $u, v \in V$, and

$$
\left|g\left(v_{0}\right)-g(v)\right|=\left|d_{X}\left(f\left(v_{0}\right), f\left(v_{0}\right)\right)-d_{X}\left(f\left(v_{0}\right), f(v)\right)\right|=d_{X}\left(f\left(v_{0}\right), f(v)\right)
$$

for any $v \in V$. Therefore,

$$
\begin{cases}|g(u)-g(v)| \leq d_{X}(f(u), f(v)), & \text { if }\{u, v\} \in E, \\ |g(u)-g(v)|=d_{X}(f(u), f(v)), & \text { if }\{u, v\} \notin E,\end{cases}
$$

for any $u, v \in V$. Thus $X$ satisfies the $G(0)$ condition.

Proposition 5.1 implies in particular that every metric space satisfies the $G(0)$ condition for every complete graph $G$.

Proposition 5.2. Let $G_{1}$ and $G_{2}$ be finite graphs, and let $G$ be the graph sum of $G_{1}$ and $G_{2}$. In other words, the vertex and edge sets of $G$ are the disjoint union of the vertex sets of $G_{1}$ and $G_{2}$ and that of the edge sets of $G_{1}$ and $G_{2}$, respectively. Suppose $X$ is a metric space that satisfies the $G_{1}(0)$ and $G_{2}(0)$ conditions. Then $X$ satisfies the $G(0)$ condition.

Proof. Suppose $G_{1}=\left(V_{1}, E_{1}\right), G_{2}=\left(V_{2}, E_{2}\right)$ and $G=(V, E)$ are finite graphs such that $V$ is the disjoint union of $V_{1}$ and $V_{2}$, and $E$ is the disjoint union of $E_{1}$ and $E_{2}$. Suppose $\left(X, d_{X}\right)$ is a metric space that satisfies the $G_{1}(0)$ and $G_{2}(0)$ conditions. Fix $f: V \rightarrow X$. Then for each $i \in\{1,2\}$, there exist a $\operatorname{CAT}(0) \operatorname{space}\left(Y_{i}, d_{Y_{i}}\right)$ and a map $g_{i}: V_{i} \rightarrow Y_{i}$ such that

$$
\begin{cases}d_{Y_{i}}\left(g_{i}(u), g_{i}(v)\right) \leq d_{X}(f(u), f(v)), & \text { if }\{u, v\} \in E_{i}, \\ d_{Y_{i}}\left(g_{i}(u), g_{i}(v)\right) \geq d_{X}(f(u), f(v)), & \text { if }\{u, v\} \notin E_{i} .\end{cases}
$$

for any $u, v \in V_{i}$. Choose vertices $v_{1} \in V_{1}$ and $v_{2} \in V_{2}$. Let

$$
d=\max \left\{d_{X}(f(u), f(v)) \mid u, v \in V\right\} .
$$


Define $\left(Y_{1}^{\prime}, d_{Y_{1}^{\prime}}\right)$ to be the metric space obtained by gluing $Y_{1}$ and the closed interval $[0, d]$ in $\mathbb{R}$ by identifying $g_{1}\left(v_{1}\right) \in Y_{1}$ with $0 \in[0, d]$. Then $\left(Y_{1}^{\prime}, d_{Y_{1}^{\prime}}\right)$ is a CAT( 0$)$ space by Reshetnyak's gluing theorem. We denote by $g_{1}^{\prime}(v)$ the point in $Y_{1}^{\prime}$ represented by $g_{1}(v) \in Y_{1}$ for each $v \in V_{1}$, and by $d^{\prime}$ the point in $Y_{1}^{\prime}$ represented by $d \in[0, d]$. Define $\left(Y, d_{Y}\right)$ to be the metric space obtained by gluing $Y_{1}^{\prime}$ and $Y_{2}$ by identifying $d^{\prime} \in Y_{1}^{\prime}$ with $g_{2}\left(v_{2}\right) \in Y_{2}$. Then $\left(Y, d_{Y}\right)$ is a CAT(0) space by Reshetnyak's gluing theorem. Define a map $g: V \rightarrow Y$ by sending each $u \in V_{1}$ to the point in $Y$ represented by $g_{1}^{\prime}(u) \in Y_{1}^{\prime}$, and each $v \in V_{2}$ the point in $Y$ represented by $g_{2}(v) \in Y_{2}$. Then

$$
\begin{aligned}
& \begin{cases}d_{Y}\left(g(u), g\left(u^{\prime}\right)\right)=d_{Y_{1}}\left(g_{1}(u), g_{1}\left(u^{\prime}\right)\right) \leq d_{X}\left(f(u), f\left(u^{\prime}\right)\right), & \text { if }\left\{u, u^{\prime}\right\} \in E_{1}, \\
d_{Y}\left(g(u), g\left(u^{\prime}\right)\right)=d_{Y_{1}}\left(g_{1}(u), g_{1}\left(u^{\prime}\right)\right) \geq d_{X}\left(f(u), f\left(u^{\prime}\right)\right), & \text { if }\left\{u, u^{\prime}\right\} \notin E_{1},\end{cases} \\
& \begin{cases}d_{Y}\left(g(v), g\left(v^{\prime}\right)\right)=d_{Y_{2}}\left(g_{2}(v), g_{2}\left(v^{\prime}\right)\right) \leq d_{X}\left(f(v), f\left(v^{\prime}\right)\right), & \text { if }\left\{v, v^{\prime}\right\} \in E_{2}, \\
d_{Y}\left(g(v), g\left(v^{\prime}\right)\right)=d_{Y_{2}}\left(g_{2}(v), g_{2}\left(v^{\prime}\right)\right) \geq d_{X}\left(f(v), f\left(v^{\prime}\right)\right), & \text { if }\left\{v, v^{\prime}\right\} \notin E_{2},\end{cases} \\
& d_{Y}(g(u), g(v))=d_{Y_{1}}\left(g_{1}(u), g_{1}\left(v_{1}\right)\right)+d+d_{Y_{2}}\left(g_{2}\left(v_{2}\right), g_{2}(v)\right) \\
& \geq d_{X}(f(u), f(v))
\end{aligned}
$$

for any $u, u^{\prime} \in V_{1}$ and any $v, v^{\prime} \in V_{2}$. It follows that

$$
\begin{cases}d_{Y}(g(u), g(v)) \leq d_{X}(f(u), f(v)), & \text { if }\{u, v\} \in E, \\ d_{Y}(g(u), g(v)) \geq d_{X}(f(u), f(v)), & \text { if }\{u, v\} \notin E\end{cases}
$$

for any $u, v \in V$. Thus $X$ satisfies the $G(0)$ condition.

Corollary 5.3. Every metric space satisfies the $G(0)$ condition for any disconnected graph $G$ with four vertices.

Proof. Let $G$ be a disconnected graph with four vertices. Then there exist graphs $G_{1}$ and $G_{2}$ such that $G$ is the graph sum of $G_{1}$ and $G_{2}$, and $G_{i}$ contains at most three vertices for each $i \in\{1,2\}$. Because every metric space that contains at most three points admits an isometric embedding into $\mathbb{R}^{2}$, every metric space satisfies the $G_{1}(0)$ and $G_{2}(0)$ conditions clearly. Therefore, it follows from Proposition 5.2 that every metric space satisfies the $G(0)$ condition.

Proposition 5.4. Let $G=(V, E)$ be a finite graph. Assume that there exist $V_{1}, V_{2} \subseteq V$ and $v_{0} \in V$ such that $V_{1} \cup V_{2}=V, V_{1} \cap V_{2}=\left\{v_{0}\right\}$, and there are no edges $\{u, v\} \in E$ with $u \in V_{1} \backslash\left\{v_{0}\right\}$ and $v \in V_{2} \backslash\left\{v_{0}\right\}$. Suppose $X$ is a metric space such that every subset $S \subseteq X$ with $|S| \leq \max \left\{\left|V_{1}\right|,\left|V_{2}\right|\right\}$ admits an isometric embedding into $a$ CAT(0) space. Then $X$ satisfies the $G(0)$ condition.

Proof. Fix a map $f: V \rightarrow X$. By hypothesis, both $f\left(V_{1}\right)$ and $f\left(V_{2}\right)$ admit isomeric embeddings into CAT(0) spaces. Hence for each $i \in\{1,2\}$, there exist a $\operatorname{CAT}(0)$ space $\left(Y_{i}, d_{Y_{i}}\right)$ and a map $g_{i}: V_{i} \rightarrow Y_{i}$ such that $d_{Y_{i}}\left(g_{i}(u), g_{i}(v)\right)=d_{X}(f(u), f(v))$ for any $u, v \in V_{i}$. Define $\left(Y, d_{Y}\right)$ to be the metric space obtained by gluing $Y_{1}$ and $Y_{2}$ by identifying $g_{1}\left(v_{0}\right) \in Y_{1}$ with $g_{2}\left(v_{0}\right) \in Y_{2}$. Then $\left(Y, d_{Y}\right)$ is a CAT(0) space by Reshetnyak's gluing theorem. Define a map $g: V \rightarrow Y$ by sending each $u \in V_{1}$ to the point in $Y$ represented by $g_{1}(u) \in Y_{1}$, and each $v \in V_{2} \backslash\left\{v_{0}\right\}$ to the point in $Y$ represented by $g_{2}(v) \in Y_{2}$. Then

$$
\begin{aligned}
d_{Y}\left(g(u), g\left(u^{\prime}\right)\right) & =d_{Y_{1}}\left(g_{1}(u), g_{1}\left(u^{\prime}\right)\right)=d_{X}\left(f(u), f\left(u^{\prime}\right)\right), \\
d_{Y}\left(g(v), g\left(v^{\prime}\right)\right) & =d_{Y_{2}}\left(g_{2}(v), g_{2}\left(v^{\prime}\right)\right)=d_{X}\left(f(v), f\left(v^{\prime}\right)\right), \\
d_{Y}(g(u), g(v)) & =d_{Y_{1}}\left(g_{1}(u), g_{1}\left(v_{0}\right)\right)+d_{Y_{2}}\left(g_{2}\left(v_{0}\right), g_{2}(v)\right) \\
& =d_{X}\left(f(u), f\left(v_{0}\right)\right)+d_{X}\left(f\left(v_{0}\right), f(v)\right) \\
& \geq d_{X}(f(u), f(v))
\end{aligned}
$$

for any $u, u^{\prime} \in V_{1}$ and $v, v^{\prime} \in V_{2}$. It follows that

$$
\begin{cases}d_{Y}(g(u), g(v))=d_{X}(f(u), f(v)), & \text { if }\{u, v\} \in E, \\ d_{Y}(g(u), g(v)) \geq d_{X}(f(u), f(v)), & \text { if }\{u, v\} \notin E\end{cases}
$$


for any $u, v \in V$. Thus $X$ satisfies the $G(0)$ condition.

For a finite graph $G=(V, E)$ and a vertex $v \in V$, the degree of $v$, denoted by $\operatorname{deg}(v)$, is the number of edges $e \in E$ such that $v \in e$.

Corollary 5.5. Suppose $G=(V, E)$ is a graph such that $|V|=4$, and there exists a vertex $v_{1} \in V$ with $\operatorname{deg}\left(v_{1}\right)=$ 1. Then every metric space satisfies the $G(0)$ condition.

Proof. Let $v_{0} \in V$ be the vertex such that $\left\{v_{0}, v_{1}\right\} \in E$. Let $V_{1}=V \backslash\left\{v_{1}\right\}$, and let $V_{2}=\left\{v_{0}, v_{1}\right\}$. Then $V_{1} \cup V_{2}=$ $V, V_{1} \cap V_{2}=\left\{v_{0}\right\}$, and there are no edges $\{u, v\} \in E$ with $u \in V_{1} \backslash\left\{v_{0}\right\}$ and $v \in V_{2} \backslash\left\{v_{0}\right\}$. Furthermore, $\max \left\{\left|V_{1}\right|,\left|V_{2}\right|\right\}=3$, and every metric space containing at most three points admits an isometric embedding into $\mathbb{R}^{2}$. Therefore, it follows from Proposition 5.4 that every metric space satisfies the $G(0)$ condition.

Recall that there are eleven simple undirected graphs on four vertices up to graph isomorphism, which are listed in FIGURE 5.1. We call them $G_{1}^{(4)}, \ldots, G_{11}^{(4)}$, respectively as in FIGURE 5.1.

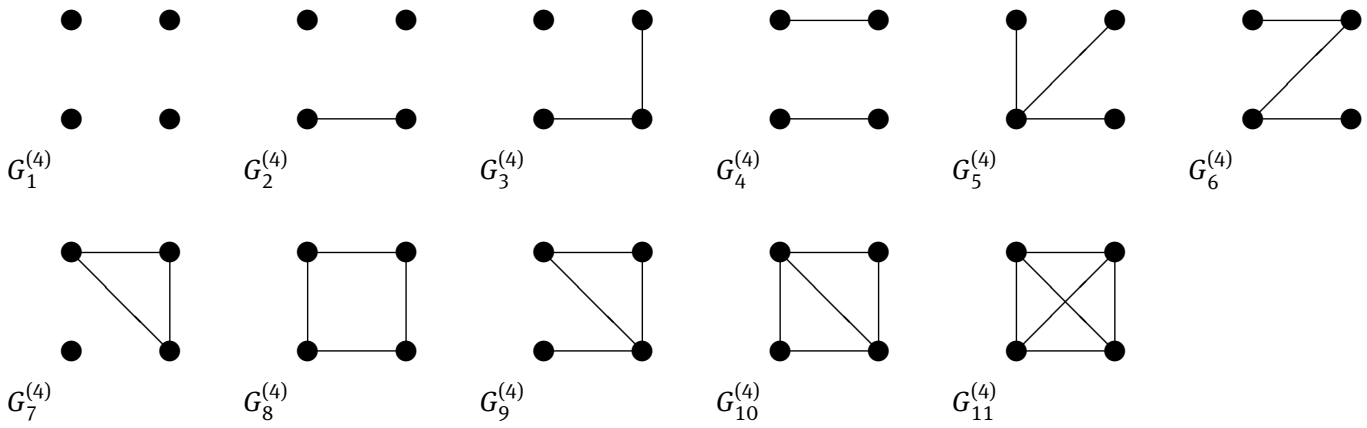

Figure 5.1: The graphs on four vertices.

All graphs listed in FIGURE 5.1 except the cycle graph $G_{8}^{(4)}$ satisfy the hypothesis of Proposition 5.1, Corollary 5.3 or Corollary 5.5. Thus every metric space satisfies the $G(0)$ conditions for all graphs $G$ with four vertices that is not isomorphic to the cycle graph. The following proposition follows from this observation and Lemma 1.12 .

Proposition 5.6. Let $V=\{1,2,3,4\}$, and let $E=\left(\begin{array}{c}V \\ 2\end{array}\right)$. Suppose $A=\left(a_{i j}\right)_{\{i, j\} \in E}$ is a family of real numbers indexed by $E$ such that every CAT(0) space satisfies the $\left(a_{i j}\right)$-quadratic metric inequality. Define $E_{+}(A) \subseteq E$ to be the set of all $\{i, j\} \in E$ with $a_{i j}>0$. If the graph $G_{A}=\left(V, E_{+}(A)\right)$ is not isomorphic to the cycle graph, then every metric space satisfies the $\left(a_{i j}\right)$-quadratic metric inequality.

Proof. If $G_{A}$ is not isomorphic to the cycle graph $G_{8}^{(4)}$, then every metric space satisfies the $G_{A}(0)$ condition as we observed above. Therefore, it follows from Lemma 1.12 that every metric space satisfies the $\left(a_{i j}\right)$-quadratic metric inequality.

It follows from the above observation and Proposition 1.9 that a four-point metric space admits an isometric embedding into a CAT(0) space if and only if it satisfies the $G_{8}^{(4)}(0)$ condition. This implies in particular that not every metric space satisfies the $G_{8}^{(4)}(0)$ condition because not every four-point metric space admits an isometric embedding into a CAT(0) space as we observed in Example 1.2. The following proposition is an immediate consequence of Theorem 1.6.

Proposition 5.7. If a metric space $X$ satisfies the $\bowtie$-inequalities, then $X$ satisfies the $G_{8}^{(4)}(0)$ condition.

Proof. If a metric space $X$ satisfies the $\otimes$-inequalities, then $X$ satisfies the $\operatorname{Cycl}_{4}(0)$ condition by Theorem 1.6, which clearly implies that $X$ satisfies the $G_{8}^{(4)}(0)$ condition. 
The facts that we have proved so far give another proof of Theorem 1.7.

Proof of Theorem 1.7. Assume that a four-point metric space $X$ admits an isometric embedding into a CAT(0) space. Then $X$ satisfies the $\otimes$-inequalities because every CAT(0) space satisfies the $\otimes$-inequalities. Conversely, assume that a four-point metric space $X$ satisfies the $\otimes$-inequalities. Then it follows from Proposition 5.1, Corollary 5.3, Corollary 5.5 and Proposition 5.7 that $X$ satisfies the $G(0)$ conditions for all graphs $G$ with four vertices, which implies that $X$ admits an isometric embedding into a CAT(0) space by Proposition 1.9.

The following facts are worth noting although they are not necessary for our purposes.

Proposition 5.8. Every metric space satisfies the $G(0)$ condition for every tree $G$.

Proof. Let $\left(X, d_{X}\right)$ be a metric space and let $G=(V, E)$ be a tree. For any $f: V \rightarrow X$, define $Y$ to be the metric tree obtained by assigning the length $d_{X}(f(u), f(v))$ to each edge $\{u, v\} \in E$ of $G$. Then $Y$ becomes a CAT(0) space, and the triangle inequality for $d_{X}$ ensures that the natural inclusion $g: V \rightarrow Y$ satisfies that

$$
\begin{cases}d_{Y}(g(u), g(v))=d_{X}(f(u), f(v)), & \text { if }\{u, v\} \in E, \\ d_{Y}(g(u), g(v)) \geq d_{X}(f(u), f(v)), & \text { if }\{u, v\} \notin E\end{cases}
$$

for any $u, v \in V$. Thus $X$ satisfies the $G(0)$ condition.

The following corollary follows immediately from Proposition 5.8 and Lemma 1.12.

Corollary 5.9. Let $n$ be a positive integer, let $V=[n]$, and let $E=\left(\begin{array}{c}V \\ 2\end{array}\right)$. Suppose $A=\left(a_{i j}\right)_{\{i, j\} \in E}$ is a family of real numbers indexed by $E$. Let $E_{+}(A)$ be the set of all $\{i, j\} \in E$ with $a_{i j}>0$. If every CAT $(0)$ space satisfies the $\left(a_{i j}\right)$-quadratic metric inequality, and if the graph $G_{A}=\left(V, E_{+}(A)\right)$ is isomorphic to a tree, then every metric space satisfies the $\left(a_{i j}\right)$-quadratic metric inequality.

\section{Five points in a $\operatorname{CAT}(0)$ space}

In this section, we prove that if a metric space $X$ satisfies the $\otimes$-inequalities, then $X$ satisfies the $G(0)$ conditions for all graphs $G$ with five vertices except two special graphs. We start with the following two propositions.

Proposition 6.1. If a metric space $X$ satisfies the $\otimes$-inequalities, then $X$ satisfies the $G(0)$ condition for every disconnected graph $G$ with five vertices.

Proof. Let $X$ be a metric space that satisfies the $\otimes$-inequalities, and let $G$ be a disconnected graph with five vertices. Then there exist graphs $G_{1}$ and $G_{2}$ such that $G$ is the graph sum of $G_{1}$ and $G_{2}$, and the number of vertices of $G_{i}$ is at most four for each $i \in\{1,2\}$. Because every subset $S \subseteq X$ with $|S| \leq 4$ admits an isometric embedding into a CAT(0) space by Theorem 1.7, $X$ satisfies the $G_{1}(0)$ and $G_{2}(0)$ conditions clearly. Thus it follows from Proposition 5.2 that $X$ satisfies the $G(0)$ condition.

Proposition 6.2. Let $X$ be a metric space that satisfies the $\nabla$-inequalities. Suppose $G=(V, E)$ is a graph such that $|V|=5$, and there exists a vertex $v_{1} \in V$ with $\operatorname{deg}\left(v_{1}\right)=1$. Then $X$ satisfies the $G(0)$ condition.

Proof. Let $v_{0} \in V$ be the vertex with $\left\{v_{0}, v_{1}\right\} \in E$, let $V_{1}=V \backslash\left\{v_{1}\right\}$, and let $V_{2}=\left\{v_{0}, v_{1}\right\}$. Then $V_{1} \cup V_{2}=V$, $V_{1} \cap V_{2}=\left\{v_{0}\right\}$, and there are no edges $\{u, v\} \in E$ with $u \in V_{1} \backslash\left\{v_{0}\right\}$ and $v \in V_{2} \backslash\left\{v_{0}\right\}$. Because $X$ satisfies the $\otimes$-inequalities, every subset $S \subseteq X$ with $|S| \leq 4$ admits an isometric embedding into a CAT(0) space by Theorem 1.7. Thus it follows from Proposition 5.4 that $X$ satisfies the $G(0)$ condition.

It follows from Proposition 6.1 and Proposition 6.2 that if a five-vertex graph $G$ has a vertex $v$ with $\operatorname{deg}(v) \leq$ 1 , then a metric space $X$ satisfies the $G(0)$ condition whenever $X$ satisfies the $\otimes$-inequalities. Up to graph 
isomorphism, there are eleven five-vertex graphs $G$ such that every vertex $v$ of $G$ satisfies $\operatorname{deg}(v) \geq 2$, which are listed in FiguRE 6.1. As in FigURE 6.1, we call these graphs $G_{1}^{(5)}, \ldots, G_{11}^{(5)}$, respectively.

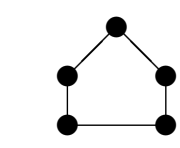

$G_{1}^{(5)}$

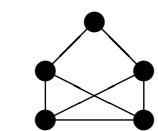

$G_{7}^{(5)}$

$$
G_{8}^{(5)}
$$
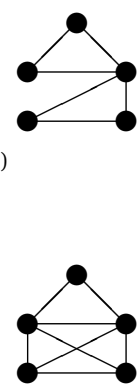

$G_{2}^{(5)}$
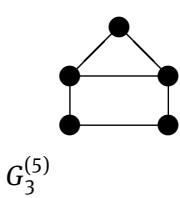

$G_{3}^{(5)}$

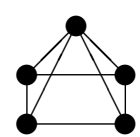

$G_{9}^{(5)}$

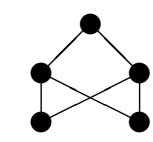

$G_{4}^{(5)}$

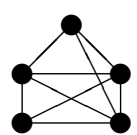

$G_{10}^{(5)}$

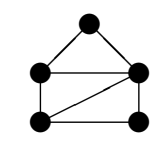

$G_{5}^{(5)}$

$G_{6}^{(5)}$

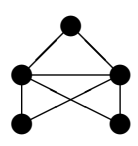

Figure 6.1: The five-vertex graphs each of whose vertex satisfies deg $\geq 2$.

Proposition 6.3. If a metric space $X$ satisfies the $\otimes$-inequalities, then $X$ satisfies the $G_{1}^{(5)}(0)$ condition.

Proof. If a metric space $X$ satisfies the $\otimes$-inequalities, then $X$ satisfies the $\operatorname{Cycl}_{5}(0)$ condition by Theorem 1.6, which clearly implies that $X$ satisfies the $G_{1}^{(5)}(0)$ condition.

Proposition 6.4. Every metric space satisfies the $G_{2}^{(5)}(0)$ condition.

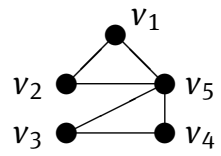

Figure 6.2

Proof. Let $V$ and $E$ be the vertex set and the edge set of $G_{2}^{(5)}(0)$, respectively. We set

$$
\begin{aligned}
& V=\left\{v_{1}, v_{2}, v_{3}, v_{4}, v_{5}\right\}, \\
& E=\left\{\left\{v_{1}, v_{2}\right\},\left\{v_{2}, v_{5}\right\},\left\{v_{5}, v_{1}\right\},\left\{v_{3}, v_{4}\right\},\left\{v_{4}, v_{5}\right\},\left\{v_{5}, v_{3}\right\}\right\},
\end{aligned}
$$

as shown in FIGURE 6.2. Set

$$
V_{1}=\left\{v_{1}, v_{2}, v_{5}\right\}, \quad V_{2}=\left\{v_{3}, v_{4}, v_{5}\right\} .
$$

Then $V_{1} \cup V_{2}=V, V_{1} \cap V_{2}=\left\{v_{5}\right\}$, and there are no edges $\{u, v\} \in E$ with $u \in V_{1} \backslash\left\{v_{5}\right\}$ and $v \in V_{2} \backslash\left\{v_{5}\right\}$. Because every metric space containing at most three points admits an isometric embedding into $\mathbb{R}^{2}$, it follows from Proposition 5.4 that every metric space satisfies the $G_{2}^{(5)}(0)$ condition.

Before proving that the validity of the $\otimes$-inequalities implies the $G_{3}^{(5)}(0)$ condition, we prove the following lemma. 
Lemma 6.5. Let $\left(X, d_{X}\right)$ be a metric space that satisfies the $\otimes$-inequalities, and let $\left(Y, d_{Y}\right)$ be a metric space. Suppose $p, x, y, z, w \in X$ and $p^{\prime}, x^{\prime}, y^{\prime}, z^{\prime}, w^{\prime} \in Y$ are points such that

$$
\begin{array}{ll}
d_{X}(p, x) \leq d_{Y}\left(p^{\prime}, x^{\prime}\right), & d_{X}(x, y) \leq d_{Y}\left(x^{\prime}, y^{\prime}\right), \quad d_{X}(y, z) \leq d_{Y}\left(y^{\prime}, z^{\prime}\right), \\
d_{X}(z, w) \leq d_{Y}\left(z^{\prime}, w^{\prime}\right), & d_{X}(w, p) \leq d_{Y}\left(w^{\prime}, p^{\prime}\right), \\
d_{X}(p, y)=d_{Y}\left(p^{\prime}, y^{\prime}\right), & d_{X}(p, z)=d_{Y}\left(p^{\prime}, z^{\prime}\right) .
\end{array}
$$

Assume that there exist subsets $T_{1}, T_{2}$ and $T_{3}$ of $Y$ that satisfy the following conditions:

(1) $T_{1}, T_{2}$ and $T_{3}$ are isometric to convex subsets of Euclidean spaces.

(2) $\left\{p^{\prime}, x^{\prime}, y^{\prime}\right\} \subseteq T_{1},\left\{p^{\prime}, y^{\prime}, z^{\prime}\right\} \subseteq T_{2}$ and $\left\{p^{\prime}, z^{\prime}, w^{\prime}\right\} \subseteq T_{3}$.

(3) There exists a geodesic segment $\Gamma_{1}$ in $Y$ with endpoints $p^{\prime}$ and $y^{\prime}$ such that

$$
\Gamma_{1} \subseteq T_{1} \cap T_{2} .
$$

(4) There exists a geodesic segment $\Gamma_{2}$ in $Y$ with endpoints $p^{\prime}$ and $z^{\prime}$ such that

$$
\Gamma_{2} \subseteq T_{2} \cap T_{3} .
$$

(5) There exist $q_{1} \in \Gamma_{1}$ and $q_{2} \in \Gamma_{2}$ such that

$$
d_{Y}\left(x^{\prime}, w^{\prime}\right)=d_{Y}\left(x^{\prime}, q_{1}\right)+d_{Y}\left(q_{1}, q_{2}\right)+d_{Y}\left(q_{2}, w^{\prime}\right) .
$$

Then $d_{X}(x, w) \leq d_{Y}\left(x^{\prime}, w^{\prime}\right)$.

Proof. Choose $p_{1}, x_{1}, y_{1}, p_{2}, y_{2}, z_{2}, p_{3}, z_{3}, w_{3} \in \mathbb{R}^{2}$ such that

$$
\begin{array}{lll}
\left\|p_{1}-x_{1}\right\|=d_{Y}\left(p^{\prime}, x^{\prime}\right), & \left\|x_{1}-y_{1}\right\|=d_{Y}\left(x^{\prime}, y^{\prime}\right), & \left\|y_{1}-p_{1}\right\|=d_{Y}\left(y^{\prime}, p^{\prime}\right), \\
\left\|p_{2}-y_{2}\right\|=d_{Y}\left(p^{\prime}, y^{\prime}\right), & \left\|y_{2}-z_{2}\right\|=d_{Y}\left(y^{\prime}, z^{\prime}\right), & \left\|z_{2}-p_{2}\right\|=d_{Y}\left(z^{\prime}, p^{\prime}\right), \\
\left\|p_{3}-z_{3}\right\|=d_{Y}\left(p^{\prime}, z^{\prime}\right), & \left\|z_{3}-w_{3}\right\|=d_{Y}\left(z^{\prime}, w^{\prime}\right), & \left\|w_{3}-p_{3}\right\|=d_{Y}\left(w^{\prime}, p^{\prime}\right) .
\end{array}
$$

Equip the subsets

$$
T_{1}^{\prime}=\operatorname{conv}\left(\left\{p_{1}, x_{1}, y_{1}\right\}\right), \quad T_{2}^{\prime}=\operatorname{conv}\left(\left\{p_{2}, y_{2}, z_{2}\right\}\right), \quad T_{3}^{\prime}=\operatorname{conv}\left(\left\{p_{3}, z_{3}, w_{3}\right\}\right) .
$$

of $\mathbb{R}^{2}$ with the induced metrics, and regard them as disjoint metric spaces. Define $\left(Y^{\prime}, d_{Y^{\prime}}\right)$ to be the metric space obtained by gluing $T_{1}^{\prime}$ and $T_{2}^{\prime}$ by identifying $\left[p_{1}, y_{1}\right] \subseteq T_{1}^{\prime}$ with $\left[p_{2}, y_{2}\right] \subseteq T_{2}^{\prime}$. Then $Y^{\prime}$ is a CAT(0) space by Reshetnyak's gluing theorem. We denote the points in $Y^{\prime}$ represented by $p_{1}, x_{1}, y_{1} \in T_{1}$ and $z_{2} \in T_{2}$ by $p^{\prime \prime}$, $x^{\prime \prime}, y^{\prime \prime}$ and $z^{\prime \prime}$, respectively. Define $\left(\tilde{Y}, d_{\tilde{Y}}\right)$ to be the metric space obtained by gluing $Y^{\prime}$ and $T_{3}^{\prime}$ by identifying $\left[p^{\prime \prime}, z^{\prime \prime}\right] \subseteq Y^{\prime}$ with $\left[p_{3}, z_{3}\right] \subseteq T_{3}$. Then $\tilde{Y}$ is a CAT(0) space by Reshetnyak's gluing theorem, which is pictured in FiguRE 6.3. We denote the points in $\tilde{Y}$ represented by $p^{\prime \prime}, x^{\prime \prime}, y^{\prime \prime}, z^{\prime \prime} \in Y^{\prime}$ and $w_{3} \in T_{3}$ by $\tilde{p}, \tilde{x}, \tilde{y}, \tilde{z}$ and $\tilde{w}$,

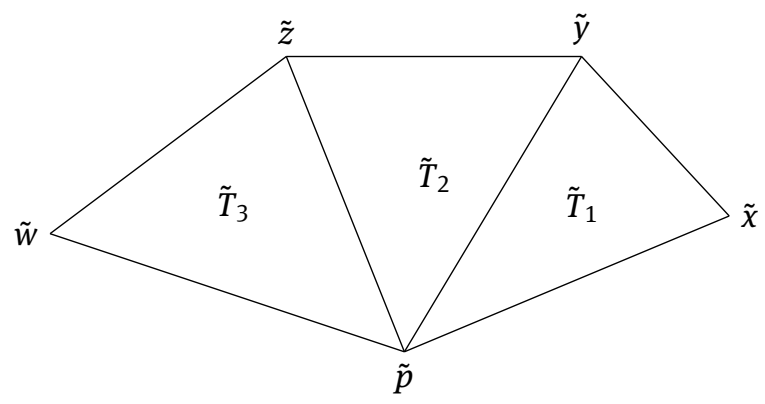

Figure 6.3: The metric space $\tilde{Y}$ in the proof of Lemma 6.5.

respectively. For each $i \in\{1,2,3\}$, the natural inclusion of $T_{i}^{\prime}$ into $\tilde{Y}$ is clearly an isometric embedding. Let 
$\tilde{T}_{i} \subseteq \tilde{Y}$ be the image of $T_{i}^{\prime}$ under the natural inclusion for each $i \in\{1,2,3\}$. It is clear from the definition of $\tilde{Y}$ that $\tilde{T}_{1} \cap \tilde{T}_{2}=[\tilde{p}, \tilde{y}]$, and $[\tilde{p}, \tilde{y}] \cap[\tilde{x}, \tilde{z}] \neq \emptyset$. Hence Lemma 3.3 implies that

$$
d_{X}(x, z) \leq d_{\tilde{Y}}(\tilde{x}, \tilde{z})
$$

because it follows from the hypothesis of the lemma and the definition of $\tilde{Y}$ that

$$
\begin{aligned}
& d_{X}(p, x) \leq d_{Y}\left(p^{\prime}, x^{\prime}\right)=d_{\tilde{Y}}(\tilde{p}, \tilde{x}), \quad d_{X}(x, y) \leq d_{Y}\left(x^{\prime}, y^{\prime}\right)=d_{\tilde{Y}}(\tilde{x}, \tilde{y}), \\
& d_{X}(y, z) \leq d_{Y}\left(y^{\prime}, z^{\prime}\right)=d_{\tilde{Y}}(\tilde{y}, \tilde{z}), \quad d_{X}(z, p)=d_{Y}\left(z^{\prime}, p^{\prime}\right)=d_{\tilde{Y}}(\tilde{z}, \tilde{p}), \\
& d_{X}(p, y)=d_{Y}\left(p^{\prime}, y^{\prime}\right)=d_{\tilde{Y}}(\tilde{p}, \tilde{y}) .
\end{aligned}
$$

Similarly, Lemma 3.3 also implies that

$$
d_{X}(y, w) \leq d_{\tilde{Y}}(\tilde{y}, \tilde{w}) .
$$

Next, we will prove that

$$
d_{X}(x, w) \leq d_{\tilde{Y}}(\tilde{x}, \tilde{w}) .
$$

To prove this, we first observe that (6.3) holds whenever one of the following equalities holds:

$$
p=x, \quad x=y, \quad y=z, \quad z=w, \quad w=p, \quad p=y, \quad p=z .
$$

If $p=x$, then $\tilde{p}=\tilde{x}$ by definition of $\tilde{Y}$, so

$$
d_{X}(x, w)=d_{X}(p, w) \leq d_{Y}\left(p^{\prime}, w^{\prime}\right)=d_{\tilde{Y}}(\tilde{p}, \tilde{w})=d_{\tilde{Y}}(\tilde{x}, \tilde{w}) .
$$

If $w=p$, then we obtain (6.3) similarly. If $x=y$, then $\tilde{x}=\tilde{y}$ by definition of $\tilde{Y}$, so it follows from (6.2) that

$$
d_{X}(x, w)=d_{X}(y, w) \leq d_{\tilde{Y}}(\tilde{y}, \tilde{w})=d_{\tilde{Y}}(\tilde{x}, \tilde{w}) .
$$

If $z=w$, then (6.3) follows from (6.1) similarly. If $p=y$ or $p=z$, then $\tilde{p} \in[\tilde{x}, \tilde{w}]$ by definition of $\tilde{Y}$, so

$$
\begin{aligned}
d_{X}(x, w) & \leq d_{X}(x, p)+d_{X}(p, w) \leq d_{Y}\left(x^{\prime}, p^{\prime}\right)+d_{Y}\left(p^{\prime}, w^{\prime}\right) \\
& =d_{\tilde{Y}}(\tilde{x}, \tilde{p})+d_{\tilde{Y}}(\tilde{p}, \tilde{w})=d_{\tilde{Y}}(\tilde{x}, \tilde{w}) .
\end{aligned}
$$

Finally, if $y=z$, then

$$
\tilde{y}=\tilde{z}, \quad \tilde{T}_{1} \cap \tilde{T}_{3}=[\tilde{p}, \tilde{y}], \quad[\tilde{p}, \tilde{y}] \cap[\tilde{x}, \tilde{w}] \neq \emptyset,
$$

by definition of $\tilde{Y}$, so Lemma 3.3 implies (6.3) because it follows from the hypothesis of the lemma and the definition of $\tilde{Y}$ that

$$
\begin{aligned}
& d_{X}(p, x) \leq d_{Y}\left(p^{\prime}, x^{\prime}\right)=d_{\tilde{Y}}(\tilde{p}, \tilde{x}), \quad d_{X}(x, y) \leq d_{Y}\left(x^{\prime}, y^{\prime}\right)=d_{\tilde{Y}}(\tilde{x}, \tilde{y}), \\
& d_{X}(y, w)=d_{X}(z, w) \leq d_{Y}\left(z^{\prime}, w^{\prime}\right)=d_{\tilde{Y}}(\tilde{z}, \tilde{w})=d_{\tilde{Y}}(\tilde{y}, \tilde{w}), \\
& d_{X}(w, p) \leq d_{Y}\left(w^{\prime}, p^{\prime}\right)=d_{\tilde{Y}}(\tilde{w}, \tilde{p}), \quad d_{X}(p, y)=d_{Y}\left(p^{\prime}, y^{\prime}\right)=d_{\tilde{Y}}(\tilde{p}, \tilde{y}) .
\end{aligned}
$$

So henceforth we assume that any equality in (6.4) does not hold. We consider four cases.

CASE 1: $\angle x_{1} y_{1} p_{1}+\angle p_{2} y_{2} z_{2} \leq \pi$ and $\angle x_{1} p_{1} y_{1}+\angle y_{2} p_{2} z_{2} \leq \pi$. In this case, the subset $\tilde{T}_{1} \cup \tilde{T}_{2}$ of $\tilde{Y}$ is isometric to a convex subset of the Euclidean plane, and it is clear from the definition of $\tilde{Y}$ that $\left(\tilde{T}_{1} \cup \tilde{T}_{2}\right) \cap \tilde{T}_{3}=[\tilde{p}, \tilde{z}]$ and $[\tilde{p}, \tilde{z}] \cap[\tilde{x}, \tilde{w}] \neq \emptyset$. Therefore, Lemma 3.3 implies the desired inequality (6.3) because it follows from the hypothesis of the lemma, the definition of $\tilde{Y}$ and (6.1) that

$$
\begin{aligned}
& d_{X}(p, x) \leq d_{Y}\left(p^{\prime}, x^{\prime}\right)=d_{\tilde{Y}}(\tilde{p}, \tilde{x}), \quad d_{X}(x, z) \leq d_{\tilde{Y}}(\tilde{x}, \tilde{z}), \\
& d_{X}(z, w) \leq d_{Y}\left(z^{\prime}, w^{\prime}\right)=d_{\tilde{Y}}(\tilde{z}, \tilde{w}), \quad d_{X}(w, p) \leq d_{Y}\left(w^{\prime}, p^{\prime}\right)=d_{\tilde{Y}}(\tilde{w}, \tilde{p}), \\
& d_{X}(p, z)=d_{Y}\left(p^{\prime}, z^{\prime}\right)=d_{\tilde{Y}}(\tilde{p}, \tilde{z}) .
\end{aligned}
$$

CASE 2: $\angle y_{2} z_{2} p_{2}+\angle p_{3} z_{3} w_{3} \leq \pi$ and $\angle y_{2} p_{2} z_{2}+\angle z_{3} p_{3} w_{3} \leq \pi$. In this case, the subset $\tilde{T}_{2} \cup \tilde{T}_{3}$ is isometric to a convex subset of the Euclidean plane, and it is clear from the definition of $\tilde{Y}$ that $\tilde{T}_{1} \cap\left(\tilde{T}_{2} \cup \tilde{T}_{3}\right)=[\tilde{p}$, $\tilde{y}]$ and $[\tilde{p}, \tilde{y}] \cap[\tilde{x}, \tilde{w}] \neq \emptyset$. Therefore, Lemma 3.3 implies the desired inequality (6.3) in the same way as in CASE 1. 
CASE 3: $\angle x_{1} p_{1} y_{1}+\angle y_{2} p_{2} z_{2}+\angle z_{3} p_{3} w_{3} \geq \pi$. In this case, we clearly have

$$
d_{\tilde{Y}}(\tilde{x}, \tilde{w})=\left\|x_{1}-p_{1}\right\|+\left\|p_{3}-w_{3}\right\|,
$$

and hence

$$
d_{\tilde{Y}}(\tilde{x}, \tilde{w})=d_{Y}\left(x^{\prime}, p^{\prime}\right)+d_{Y}\left(p^{\prime}, w^{\prime}\right) \geq d_{X}(x, p)+d_{X}(p, w) \geq d_{X}(x, w) .
$$

CASE 4: Neither the assumption of CASE 1, CASE 2 nor CASE 3 holds. In this case,

$$
\angle x_{1} p_{1} y_{1}+\angle y_{2} p_{2} z_{2} \leq \pi, \quad \angle y_{2} p_{2} z_{2}+\angle z_{3} p_{3} w_{3} \leq \pi
$$

because the assumption of CASE 3 does not hold. Because neither the assumption of CASE 1 nor CASE 2 holds, it follows from (6.5) that

$$
\angle x_{1} y_{1} p_{1}+\angle p_{2} y_{2} z_{2}>\pi, \quad \angle y_{2} z_{2} p_{2}+\angle p_{3} z_{3} w_{3}>\pi
$$

It clearly follows from (6.6) that

$$
d_{\tilde{Y}}(\tilde{x}, \tilde{w})=\left\|x_{1}-y_{1}\right\|+\left\|y_{2}-z_{2}\right\|+\left\|z_{3}-w_{3}\right\|,
$$

and hence

$$
\begin{aligned}
d_{\tilde{Y}}(\tilde{x}, \tilde{w}) & =d_{Y}\left(x^{\prime}, y^{\prime}\right)+d_{Y}\left(y^{\prime}, z^{\prime}\right)+d_{Y}\left(z^{\prime}, w^{\prime}\right) \\
& \geq d_{X}(x, y)+d_{X}(y, z)+d_{X}(z, w) \geq d_{X}(x, w),
\end{aligned}
$$

which completes the proof of (6.3).

It follows from the conditions (1) and (2) in the statement of the lemma that there exist isometric embeddings $f_{1}: \tilde{T}_{1} \rightarrow T_{1}, f_{2}: \tilde{T}_{2} \rightarrow T_{2}$ and $f_{3}: \tilde{T}_{3} \rightarrow T_{3}$ such that

$$
\begin{array}{lll}
f_{1}(\tilde{p})=p^{\prime}, & f_{1}(\tilde{x})=x^{\prime}, & f_{1}(\tilde{y})=y^{\prime} \\
f_{2}(\tilde{p})=p^{\prime}, & f_{2}(\tilde{y})=y^{\prime}, & f_{2}(\tilde{z})=z^{\prime} \\
f_{3}(\tilde{p})=p^{\prime}, & f_{3}(\tilde{z})=z^{\prime}, & f_{3}(\tilde{w})=w^{\prime} .
\end{array}
$$

Then $f_{1}([\tilde{p}, \tilde{y}])$ is a geodesic segment with endpoints $p^{\prime}$ and $y^{\prime}$ contained in $T_{1}$, and $f_{2}([\tilde{p}, \tilde{y}])$ is a geodesic segment with endpoints $p^{\prime}$ and $y^{\prime}$ contained in $T_{2}$. Since $T_{1}$ and $T_{2}$ are both uniquely geodesic, it follows that $f_{1}([\tilde{p}, \tilde{y}])=\Gamma_{1}=f_{2}([\tilde{p}, \tilde{y}])$, and thus $f_{1}$ and $f_{2}$ agree on $[\tilde{p}, \tilde{y}]$. Similarly, $f_{2}$ and $f_{3}$ agree on $[\tilde{p}, \tilde{z}]$. Suppose $\tilde{q}_{1} \in[\tilde{p}, \tilde{y}]$ and $\tilde{q}_{2} \in[\tilde{p}, \tilde{z}]$ are the points such that $f_{1}\left(\tilde{q}_{1}\right)=f_{2}\left(\tilde{q}_{1}\right)=q_{1}$ and $f_{2}\left(\tilde{q}_{2}\right)=f_{3}\left(\tilde{q}_{2}\right)=q_{2}$. Then

$$
\begin{aligned}
d_{Y}\left(x^{\prime}, w^{\prime}\right) & =d_{Y}\left(x^{\prime}, q_{1}\right)+d_{Y}\left(q_{1}, q_{2}\right)+d_{Y}\left(q_{2}, w^{\prime}\right) \\
& =d_{\tilde{Y}}\left(f_{1}^{-1}\left(x^{\prime}\right), f_{1}^{-1}\left(q_{1}\right)\right)+d_{\tilde{Y}}\left(f_{2}^{-1}\left(q_{1}\right), f_{2}^{-1}\left(q_{2}\right)\right)+d_{\tilde{Y}}\left(f_{3}^{-1}\left(q_{2}\right), f_{3}^{-1}\left(w^{\prime}\right)\right) \\
& =d_{\tilde{Y}}\left(\tilde{x}, \tilde{q}_{1}\right)+d_{\tilde{Y}}\left(\tilde{q}_{1}, \tilde{q}_{2}\right)+d_{\tilde{Y}}\left(\tilde{q}_{2}, \tilde{w}\right) \geq d_{\tilde{Y}}(\tilde{x}, \tilde{w}) .
\end{aligned}
$$

Combining this with (6.3) yields $d_{X}(x, w) \leq d_{Y}\left(x^{\prime}, w^{\prime}\right)$.

Proposition 6.6. If a metric space $X$ satisfies the $\otimes$-inequalities, then $X$ satisfies the $G_{3}^{(5)}(0)$ and $G_{5}^{(5)}(0)$ conditions.

Proof. Let $\left(X, d_{X}\right)$ be a metric space that satisfies the $\otimes$-inequalities. Suppose the graphs $G_{3}^{(5)}$ and $G_{5}^{(5)}$ have a common vertex set $V$, and edge sets $E_{3}$ and $E_{5}$, respectively. We set

$$
\begin{aligned}
V & =\left\{v_{1}, v_{2}, v_{3}, v_{4}, v_{5}\right\}, \\
E_{3} & =\left\{\left\{v_{1}, v_{2}\right\},\left\{v_{2}, v_{3}\right\},\left\{v_{3}, v_{4}\right\},\left\{v_{4}, v_{5}\right\},\left\{v_{5}, v_{1}\right\},\left\{v_{2}, v_{5}\right\}\right\}, \\
E_{5} & =\left\{\left\{v_{1}, v_{2}\right\},\left\{v_{2}, v_{3}\right\},\left\{v_{3}, v_{4}\right\},\left\{v_{4}, v_{5}\right\},\left\{v_{5}, v_{1}\right\},\left\{v_{2}, v_{5}\right\},\left\{v_{3}, v_{5}\right\}\right\},
\end{aligned}
$$



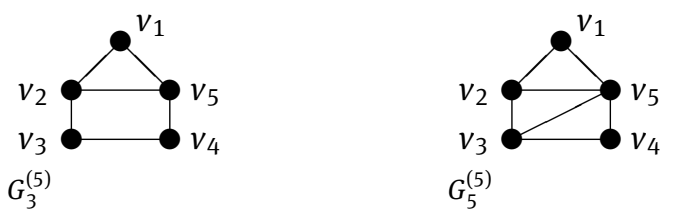

Figure 6.4

as shown in FIGURE 6.4. Fix a map $f: V \rightarrow X$, and set

$$
d_{i j}=d_{X}\left(f\left(v_{i}\right), f\left(v_{j}\right)\right)
$$

for any $i, j \in\{1,2,3,4,5\}$. By Theorem 1.7, if $d_{i j}=0$ for some $i, j \in\{1,2,3,4,5\}$ with $i \neq j$, then there exist a CAT(0) space $\left(Y_{0}, d_{Y_{0}}\right)$ and a map $g_{0}: V \rightarrow Y_{0}$ such that $d_{Y_{0}}\left(g_{0}\left(v_{i}\right), g_{0}\left(v_{j}\right)\right)=d_{i j}$ for any $i, j \in\{1,2,3,4,5\}$. Hence we assume $d_{i j}>0$ for any $i, j \in\{1,2,3,4,5\}$ with $i \neq j$.

Choose $x_{1}, x_{2}, x_{5}, y_{2}, y_{3}, y_{5}, z_{3}, z_{4}, z_{5} \in \mathbb{R}^{2}$ such that

$$
\begin{array}{lll}
\left\|x_{1}-x_{2}\right\|=d_{12}, & \left\|x_{2}-x_{5}\right\|=d_{25}, & \left\|x_{5}-x_{1}\right\|=d_{51}, \\
\left\|y_{2}-y_{3}\right\|=d_{23}, & \left\|y_{3}-y_{5}\right\|=d_{35}, & \left\|y_{5}-y_{2}\right\|=d_{52}, \\
\left\|z_{3}-z_{4}\right\|=d_{34}, & \left\|z_{4}-z_{5}\right\|=d_{45}, & \left\|z_{5}-z_{3}\right\|=d_{53} .
\end{array}
$$

Equip the subsets

$$
T_{1}=\operatorname{conv}\left(\left\{x_{1}, x_{2}, x_{5}\right\}\right), \quad T_{2}=\operatorname{conv}\left(\left\{y_{2}, y_{3}, y_{5}\right\}\right), \quad T_{3}=\operatorname{conv}\left(\left\{z_{3}, z_{4}, z_{5}\right\}\right)
$$

of $\mathbb{R}^{2}$ with the induced metrics, and regard them as disjoint metric spaces. Define $\left(Y^{\prime}, d_{Y^{\prime}}\right)$ to be the metric space obtained by gluing $T_{1}$ and $T_{2}$ by identifying $\left[x_{2}, x_{5}\right] \subseteq T_{1}$ with $\left[y_{2}, y_{5}\right] \subseteq T_{2}$. Then $\left(Y^{\prime}, d_{Y^{\prime}}\right)$ is a CAT(0) space by Reshetnyak's gluing theorem. We denote by $p_{i}$ the point in $Y^{\prime}$ represented by $x_{i} \in T_{1}$ for each $i \in\{1,2,5\}$, and by $p_{3}$ the point in $Y^{\prime}$ represented by $y_{3} \in T_{2}$. Define $\left(Y, d_{Y}\right)$ to be the metric space obtained by gluing $Y^{\prime}$ and $T_{3}$ by identifying $\left[p_{3}, p_{5}\right] \subseteq Y^{\prime}$ with $\left[z_{3}, z_{5}\right] \subseteq T_{3}$. Then $\left(Y, d_{Y}\right)$ is a $\operatorname{CAT}(0)$ space by Reshetnyak's gluing theorem, and for each $i \in\{1,2,3\}$, the natural inclusion of $T_{i}$ into $Y$ is clearly an isometric embedding. Let $g: V \rightarrow Y$ be the map that assigns the point in $Y$ represented by $p_{i} \in Y^{\prime}$ to $v_{i} \in V$ for each $i \in\{1,2,3,5\}$, and the point in $Y$ represented by $z_{4} \in T_{3}$ to $v_{4} \in V$. Then Lemma 3.3 implies that

$$
d_{Y}\left(g\left(v_{1}\right), g\left(v_{3}\right)\right) \geq d_{13}, \quad d_{Y}\left(g\left(v_{2}\right), g\left(v_{4}\right)\right) \geq d_{24},
$$

and Lemma 6.5 implies that

$$
d_{Y}\left(g\left(v_{1}\right), g\left(v_{4}\right)\right) \geq d_{14} .
$$

It follows from (6.7), (6.8) and the definition of $Y$ that any $u, v \in V$ satisfy

$$
\begin{cases}d_{Y}(g(u), g(v))=d_{X}(f(u), f(v)), & \text { if }\{u, v\} \in E_{i}, \\ d_{Y}(g(u), g(v)) \geq d_{X}(f(u), f(v)), & \text { if }\{u, v\} \notin E_{i}\end{cases}
$$

for each $i \in\{3,5\}$. Thus $X$ satisfies the $G_{3}^{(5)}(0)$ and $G_{5}^{(5)}(0)$ conditions.

Proposition 6.7. If a metric space $X$ satisfies the $\otimes$-inequalities, then $X$ satisfies the $G_{4}^{(5)}(0)$ and $G_{6}^{(5)}(0)$ conditions.

Proof. Let $\left(X, d_{X}\right)$ be a metric space that satisfies the $\otimes$-inequalities. Suppose the graphs $G_{4}^{(5)}$ and $G_{6}^{(5)}$ have a common vertex set $V$, and edge sets $E_{4}$ and $E_{6}$, respectively. We set

$$
\begin{aligned}
V & =\left\{v_{1}, v_{2}, v_{3}, v_{4}, v_{5}\right\}, \\
E_{4} & =\left\{\left\{v_{1}, v_{2}\right\},\left\{v_{2}, v_{3}\right\},\left\{v_{4}, v_{5}\right\},\left\{v_{5}, v_{1}\right\},\left\{v_{2}, v_{4}\right\},\left\{v_{3}, v_{5}\right\}\right\}, \\
E_{6} & =\left\{\left\{v_{1}, v_{2}\right\},\left\{v_{2}, v_{3}\right\},\left\{v_{4}, v_{5}\right\},\left\{v_{5}, v_{1}\right\},\left\{v_{2}, v_{4}\right\},\left\{v_{3}, v_{5}\right\},\left\{v_{5}, v_{2}\right\}\right\},
\end{aligned}
$$



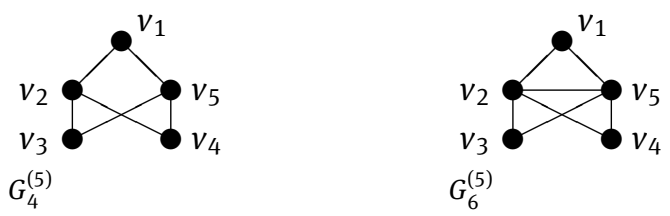

Figure 6.5

as shown in FIGURE 6.5. Fix a map $f: V \rightarrow X$, and set

$$
d_{i j}=d_{X}\left(f\left(v_{i}\right), f\left(v_{j}\right)\right)
$$

for any $i, j \in\{1,2,3,4,5\}$. By Theorem 1.7 , if $d_{i j}=0$ for some $i, j \in\{1,2,3,4,5\}$ with $i \neq j$, then there exist $\operatorname{aCAT}(0) \operatorname{space}\left(Y_{0}, d_{Y_{0}}\right)$ and a map $g_{0}: V \rightarrow Y_{0}$ such that $d_{Y_{0}}\left(g_{0}\left(v_{i}\right), g_{0}\left(v_{j}\right)\right)=d_{i j}$ for any $i, j \in\{1,2,3,4,5\}$. Hence we assume $d_{i j}>0$ for any $i, j \in\{1,2,3,4,5\}$ with $i \neq j$.

Choose $x_{1}, x_{2}, x_{5}, y_{2}, y_{3}, y_{5}, z_{2}, z_{4}, z_{5} \in \mathbb{R}^{2}$ such that

$$
\begin{array}{lll}
\left\|x_{1}-x_{2}\right\|=d_{12}, & \left\|x_{2}-x_{5}\right\|=d_{25}, & \left\|x_{5}-x_{1}\right\|=d_{51}, \\
\left\|y_{2}-y_{3}\right\|=d_{23}, & \left\|y_{3}-y_{5}\right\|=d_{35}, & \left\|y_{5}-y_{2}\right\|=d_{52}, \\
\left\|z_{2}-z_{4}\right\|=d_{24}, & \left\|z_{4}-z_{5}\right\|=d_{45}, & \left\|z_{5}-z_{2}\right\|=d_{52} .
\end{array}
$$

Equip the subsets

$$
T_{1}=\operatorname{conv}\left(\left\{x_{1}, x_{2}, x_{5}\right\}\right), \quad T_{2}=\operatorname{conv}\left(\left\{y_{2}, y_{3}, y_{5}\right\}\right), \quad T_{3}=\operatorname{conv}\left(\left\{z_{2}, z_{4}, z_{5}\right\}\right)
$$

of $\mathbb{R}^{2}$ with the induced metrics, and regard them as disjoint metric spaces. We define $Y^{\prime}$ to be the metric space obtained by gluing $T_{1}$ and $T_{2}$ by identifying $\left[x_{2}, x_{5}\right] \subseteq T_{1}$ with $\left[y_{2}, y_{5}\right] \subseteq T_{2}$. Then $Y^{\prime}$ is a CAT(0) space by Reshetnyak's gluing theorem. We denote by $p_{i}$ the point in $Y^{\prime}$ represented by $x_{i} \in T_{1}$ for each $i \in\{1,2,5\}$, and by $p_{3}$ the point in $Y^{\prime}$ represented by $y_{3} \in T_{2}$. Define $\left(Y, d_{Y}\right)$ to be the metric space obtained by gluing $Y^{\prime}$ and $T_{3}$ by identifying $\left[p_{2}, p_{5}\right] \subseteq Y^{\prime}$ with $\left[z_{2}, z_{5}\right] \subseteq T_{3}$. Then $\left(Y, d_{Y}\right)$ is a CAT( 0$)$ space by Reshetnyak's gluing theorem, and the natural inclusion of $T_{i}$ into $Y$ is clearly an isometric embedding for each $i \in\{1,2,3\}$. Let $g: V \rightarrow Y$ be the map that assigns the point in $Y$ represented by $p_{i} \in Y^{\prime}$ to each $v_{i} \in\left\{v_{1}, v_{2}, v_{3}, v_{5}\right\}$, and the point in $Y$ represented by $z_{4} \in T_{3}$ to $v_{4}$. Then it is clear from the definition of $Y$ that the geodesic segment $\left[g\left(v_{2}\right), g\left(v_{5}\right)\right] \subseteq Y$ is shared by the images of $T_{1}, T_{2}$ and $T_{3}$ under the natural inclusions. Because it is also clear from the definition of $Y$ that $\left[g\left(v_{2}\right), g\left(v_{5}\right)\right] \cap\left[g\left(v_{1}\right), g\left(v_{3}\right)\right] \neq \emptyset$, Lemma 3.3 implies that

$$
d_{Y}\left(g\left(v_{1}\right), g\left(v_{3}\right)\right) \geq d_{13} .
$$

Similarly, Lemma 3.3 also implies that

$$
d_{Y}\left(g\left(v_{3}\right), g\left(v_{4}\right)\right) \geq d_{34}, \quad d_{Y}\left(g\left(v_{4}\right), g\left(v_{1}\right)\right) \geq d_{41} .
$$

It follows from (6.9), (6.10) and the definition of $Y$ that any $u, v \in V$ satisfy

$$
\begin{cases}d_{Y}(g(u), g(v))=d_{X}(f(u), f(v)), & \text { if }\{u, v\} \in E_{i}, \\ d_{Y}(g(u), g(v)) \geq d_{X}(f(u), f(v)), & \text { if }\{u, v\} \notin E_{i},\end{cases}
$$

for each $i \in\{4,6\}$. Thus $X$ satisfies the $G_{4}^{(5)}(0)$ and $G_{6}^{(5)}(0)$ conditions.

The following proposition follows immediately from Proposition 5.1.

Proposition 6.8. Every metric space satisfies the $G_{8}^{(5)}(0), G_{10}^{(5)}(0)$ and $G_{11}^{(5)}(0)$ conditions.

Proof. For each $i \in\{8,10,11\}$, the graph $G_{i}^{(5)}=(V, E)$ has a vertex $v_{0} \in V$ such that $\{u, v\} \in E$ for any $u, v \in V \backslash\left\{v_{0}\right\}$ with $u \neq v$. Therefore, Proposition 5.1 implies that every metric space satisfies the $G_{i}^{(5)}(0)$ condition for each $i \in\{8,10,11\}$. 
By Propositions 6.1, 6.2, 6.3, 6.4, 6.6, 6.7 and 6.8, to prove that the validity of the $\otimes$-inequalities implies the $G(0)$ condition for every graph $G$ with five vertices, it only remains to prove that it implies the $G_{7}^{(5)}(0)$ and $G_{9}^{(5)}(0)$ conditions.

\section{Embeddability of four points into a Euclidean space}

In this section, we introduce certain concepts concerning isometric embeddability of four-point subsets of metric spaces into the three dimensional Euclidean space, and by using those concepts, discuss several properties of metric spaces that satisfy the $\otimes$-inequalities. Those properties will be used to prove that the validity of the $\otimes$-inequalities implies the $G_{7}^{(5)}(0)$ and $G_{9}^{(5)}(0)$ conditions.

Definition 7.1. Let $\left(X, d_{X}\right)$ be a metric space, and let $x, y, z, w \in X$ be four distinct points. We say that $\{x, y, z, w\}$ is under-distance (resp. over-distance) with respect to $\{y, w\}$ if any $x^{\prime}, y^{\prime}, z^{\prime}, w^{\prime} \in \mathbb{R}^{3}$ satisfy $d_{X}(y, w)<\left\|y^{\prime}-w^{\prime}\right\|$ (resp. $\left.\left\|y^{\prime}-w^{\prime}\right\|<d_{X}(y, w)\right)$ whenever

$$
\begin{aligned}
&\left\|x^{\prime}-y^{\prime}\right\|=d_{X}(x, y), \quad\left\|y^{\prime}-z^{\prime}\right\|=d_{X}(y, z), \quad\left\|z^{\prime}-x^{\prime}\right\|=d_{X}(z, x), \\
&\left\|x^{\prime}-w^{\prime}\right\|=d_{X}(x, w), \quad\left\|w^{\prime}-z^{\prime}\right\|=d_{X}(w, z) .
\end{aligned}
$$

It is easily observed that for any four distinct points $x y, z$ and $w$ in any metric space $X$, there exist $x^{\prime}, y^{\prime}, z^{\prime}, w^{\prime} \in \mathbb{R}^{3}$ satisfying (7.1). Therefore, $\{x, y, z, w\}$ does not become under-distance and over-distance with respect to $\{y, w\}$ simultaneously.

Proposition 7.2. Let $\left(X, d_{X}\right)$ be a metric space, and let $x, y, z, w \in X$ be four distinct points. Then one and only one of the following conditions holds true.

(a) The subset $\{x, y, z, w\} \subseteq X$ admits an isometric embedding into $\mathbb{R}^{3}$.

(b) $\{x, y, z, w\}$ is under-distance with respect to $\{y, w\}$.

(c) $\{x, y, z, w\}$ is over-distance with respect to $\{y, w\}$.

Proof. Define $\tilde{x}, \tilde{z} \in \mathbb{R}^{3}$ by

$$
\tilde{x}=(0,0,0), \quad \tilde{z}=\left(d_{X}(x, z), 0,0\right) .
$$

Suppose $\tilde{y}=\left(y^{(1)}, y^{(2)}, 0\right)$ and $\tilde{w}=\left(w^{(1)}, w^{(2)}, 0\right)$ are the points in $\mathbb{R}^{3}$ such that

$$
\begin{aligned}
& \|\tilde{x}-\tilde{y}\|=d_{X}(x, y), \quad\|\tilde{y}-\tilde{z}\|=d_{X}(y, z), \quad y^{(2)} \geq 0, \\
& \|\tilde{x}-\tilde{w}\|=d_{X}(x, w), \quad\|\tilde{w}-\tilde{z}\|=d_{X}(w, z), \quad w^{(2)} \geq 0 .
\end{aligned}
$$

Clearly, such $\tilde{y}$ and $\tilde{w}$ exist uniquely. For each $\theta \in[0, \pi]$, define $\tilde{w}(\theta) \in \mathbb{R}^{3}$ by

$$
\tilde{w}(\theta)=\left(w^{(1)}, w^{(2)} \cos \theta, w^{(2)} \sin \theta\right) .
$$

Then it is easily seen that

$$
\|\tilde{X}-\tilde{w}(\theta)\|=d_{X}(x, w), \quad\|\tilde{w}(\theta)-\tilde{z}\|=d_{X}(w, z)
$$

for any $\theta \in[0, \pi]$, and the function $\theta \mapsto\|\tilde{y}-\tilde{w}(\theta)\|$ is non-decreasing on $[0, \pi]$. To prove the proposition, it suffices to prove the following three statements:

$\left(\mathrm{a}^{\prime}\right)\{x, y, z, w\}$ admits an isometric embedding into $\mathbb{R}^{3}$ if and only if

$$
\|\tilde{y}-\tilde{w}(0)\| \leq d_{X}(y, w) \leq\|\tilde{y}-\tilde{w}(\pi)\| \text {. }
$$

(b') $\{x, y, z, w\}$ is under-distance with respect to $\{y, w\}$ if and only if

$$
d_{X}(y, w)<\|\tilde{y}-\tilde{w}(0)\| \text {. }
$$


$\left(c^{\prime}\right)\{x, y, z, w\}$ is over-distance with respect to $\{y, w\}$ if and only if

$$
\|\tilde{y}-\tilde{w}(\pi)\|<d_{X}(y, w) .
$$

Let $x^{\prime}, y^{\prime}, z^{\prime}, w^{\prime} \in \mathbb{R}^{3}$ be arbitrary points that satisfy the equalities (7.1) in Definition 7.1. Then there exists a point $w^{\prime \prime}=\left(\alpha_{1}, \alpha_{2}, \alpha_{3}\right) \in \mathbb{R}^{3}$ such that

$$
\left\|x^{\prime}-w^{\prime}\right\|=\left\|\tilde{x}-w^{\prime \prime}\right\|, \quad\left\|y^{\prime}-w^{\prime}\right\|=\left\|\tilde{y}-w^{\prime \prime}\right\|, \quad\left\|z^{\prime}-w^{\prime}\right\|=\left\|\tilde{z}-w^{\prime \prime}\right\|
$$

by definition of the points $\tilde{x}, \tilde{y}$ and $\tilde{z}$. Then

$$
\begin{aligned}
\alpha_{1}^{2}+\alpha_{2}^{2}+\alpha_{3}^{2} & =\left(w^{(1)}\right)^{2}+\left(w^{(2)}\right)^{2}, \\
\left(\alpha_{1}-d_{X}(x, z)\right)^{2}+\alpha_{2}^{2}+\alpha_{3}^{2} & =\left(w^{(1)}-d_{X}(x, z)\right)^{2}+\left(w^{(2)}\right)^{2}
\end{aligned}
$$

because

$$
\left\|\tilde{x}-w^{\prime \prime}\right\|=d_{X}(x, w)=\|\tilde{x}-\tilde{w}\|, \quad\left\|w^{\prime \prime}-\tilde{z}\right\|=d_{X}(w, z)=\|\tilde{w}-\tilde{z}\| .
$$

Since $d_{X}(x, z) \neq 0$, these equalities imply that

$$
\alpha_{1}=w^{(1)}, \quad \alpha_{2}^{2}+\alpha_{3}^{2}=\left(w^{(2)}\right)^{2} .
$$

It follows from the second equality in (7.3) that

$$
\left|\alpha_{2}\right| \leq\left|w^{(2)}\right|
$$

Using (7.3), we compute that

$$
\begin{aligned}
\left\|y^{\prime}-w^{\prime}\right\|^{2} & =\left\|\tilde{y}-w^{\prime \prime}\right\|^{2} \\
& =\left(y^{(1)}-\alpha_{1}\right)^{2}+\left(y^{(2)}-\alpha_{2}\right)^{2}+\alpha_{3}^{2} \\
& =\left(y^{(1)}-w^{(1)}\right)^{2}+\left(y^{(2)}\right)^{2}-2 \alpha_{2} y^{(2)}+\left(w^{(2)}\right)^{2} .
\end{aligned}
$$

Together with (7.4), this implies that

$$
\|\tilde{y}-\tilde{w}(0)\| \leq\left\|y^{\prime}-w^{\prime}\right\| \leq\|\tilde{y}-\tilde{w}(\pi)\|
$$

because

$$
\|\tilde{y}-\tilde{w}(0)\|^{2}=\left(y^{(1)}-w^{(1)}\right)^{2}+\left(y^{(2)}-w^{(2)}\right)^{2}, \quad\|\tilde{y}-\tilde{w}(\pi)\|^{2}=\left(y^{(1)}-w^{(1)}\right)^{2}+\left(y^{(2)}+w^{(2)}\right)^{2} .
$$

The statements $\left(\mathrm{b}^{\prime}\right)$ and $\left(\mathrm{c}^{\prime}\right)$ follow immediately from the fact that the inequality (7.5) holds true for arbitrary $x^{\prime}, y^{\prime}, z^{\prime}, w^{\prime} \in \mathbb{R}^{3}$ satisfying (7.1). It also follows immediately from this fact that if $\{x, y, z, w\}$ admits an isometric embedding into $\mathbb{R}^{3}$, then (7.2) holds true. If (7.2) holds true, then there exists $\theta_{0} \in[0, \pi]$ that satisfies

$$
\left\|\tilde{y}-\tilde{w}\left(\theta_{0}\right)\right\|=d_{X}(y, w)
$$

because the function $\theta \mapsto\|\tilde{y}-\tilde{w}(\theta)\|$ is continuous on $[0, \pi]$, and therefore the map $\varphi:\{x, y, z, w\} \rightarrow \mathbb{R}^{3}$ defined by

$$
\varphi(x)=\tilde{x}, \quad \varphi(y)=\tilde{y}, \quad \varphi(z)=\tilde{z}, \quad \varphi(w)=\tilde{w}\left(\theta_{0}\right)
$$

is an isometric embedding. Thus $\left(\mathrm{a}^{\prime}\right)$ is also true.

Before discussing properties of metric spaces that satisfy the $\otimes$-inequalities by using the concepts introduced above, we recall the following two basic facts. Both of them hold clearly, so we omit their proofs.

Lemma 7.3. Suppose $x, y, z, x^{\prime}, y^{\prime}, z^{\prime} \in \mathbb{R}^{2}$ are points such that

$$
0<\|x-y\|=\left\|x^{\prime}-y^{\prime}\right\|, \quad 0<\|z-y\|=\left\|z^{\prime}-y^{\prime}\right\| .
$$

Then $\|x-z\| \leq\left\|x^{\prime}-z^{\prime}\right\|$ if and only if $\angle x y z \leq \angle x^{\prime} y^{\prime} z^{\prime}$. Moreover, $\|x-z\|=\left\|x^{\prime}-z^{\prime}\right\|$ if and only if $\angle x y z=\angle x^{\prime} y^{\prime} z^{\prime}$. 
Lemma 7.4. Suppose $x, y, z, w \in \mathbb{R}^{2}$ are points such that $w \notin\{x, y, z\}$. Then

$$
w \in \operatorname{conv}(\{x, y, z\})
$$

if and only if $y$ and $z$ are not on the same side of $\overleftrightarrow{x w}$, and $\pi \leq \angle y w x+\angle x w z$

In the rest of this section, we discuss several properties of metric spaces that satisfy the $\otimes$-inequalities by using the concepts introduced above.

Lemma 7.5. Let $\left(X, d_{X}\right)$ be a metric space that satisfies the $\otimes$-inequalities. Suppose $x, y, z, w \in X$ are four distinct points such that $\{x, y, z, w\}$ is under-distance with respect to $\{y, w\}$. Suppose $x^{\prime}, y^{\prime}, z^{\prime}, w^{\prime} \in \mathbb{R}^{2}$ are points such that

$$
\begin{aligned}
& \left\|x^{\prime}-y^{\prime}\right\|=d_{X}(x, y), \quad\left\|y^{\prime}-z^{\prime}\right\|=d_{X}(y, z), \quad\left\|z^{\prime}-x^{\prime}\right\|=d_{X}(z, x) \\
& \left\|x^{\prime}-w^{\prime}\right\|=d_{X}(x, w), \quad\left\|w^{\prime}-z^{\prime}\right\|=d_{X}(w, z) .
\end{aligned}
$$

Then

$$
\left[x^{\prime}, y^{\prime}\right] \cap\left[z^{\prime}, w^{\prime}\right]=\emptyset, \quad\left[x^{\prime}, w^{\prime}\right] \cap\left[y^{\prime}, z^{\prime}\right]=\emptyset,
$$

and the points $x^{\prime}, y^{\prime}, z^{\prime}$ and $w^{\prime}$ are not collinear.

Proof. If we had $\left[x^{\prime}, y^{\prime}\right] \cap\left[z^{\prime}, w^{\prime}\right] \neq \emptyset$, then Lemma 3.5 would imply that

$$
\left\|y^{\prime}-w^{\prime}\right\| \leq d_{X}(y, w)
$$

contradicting the hypothesis that $\{x, y, z, w\}$ is under-distance with respect to $\{y, w\}$. Hence we have

$$
\left[x^{\prime}, y^{\prime}\right] \cap\left[z^{\prime}, w^{\prime}\right]=\emptyset .
$$

Similarly, we also have

$$
\left[x^{\prime}, w^{\prime}\right] \cap\left[y^{\prime}, z^{\prime}\right]=\emptyset .
$$

To prove that $x^{\prime}, y^{\prime}, z^{\prime}$ and $w^{\prime}$ are not collinear, suppose to the contrary that there exists a straight line $L \subset \mathbb{R}^{2}$ containing $x^{\prime}, y^{\prime}, z^{\prime}$ and $w^{\prime}$. Choose an isometric embedding $\varphi: L \rightarrow \mathbb{R}$ such that $\varphi\left(x^{\prime}\right)<\varphi\left(z^{\prime}\right)$. Define maps $\gamma_{1}:[0,2] \rightarrow \mathbb{R}, \gamma_{2}:[0,2] \rightarrow \mathbb{R}$ and $f:[0,2] \rightarrow \mathbb{R}$ by

$$
\begin{aligned}
& \gamma_{1}(t)= \begin{cases}\varphi\left(x^{\prime}\right)+\left(\varphi\left(y^{\prime}\right)-\varphi\left(x^{\prime}\right)\right) t, & t \in[0,1], \\
\varphi\left(y^{\prime}\right)+\left(\varphi\left(z^{\prime}\right)-\varphi\left(y^{\prime}\right)\right)(t-1), & t \in(1,2],\end{cases} \\
& \gamma_{2}(t)= \begin{cases}\varphi\left(z^{\prime}\right)+\left(\varphi\left(w^{\prime}\right)-\varphi\left(z^{\prime}\right)\right) t, & t \in[0,1], \\
\varphi\left(w^{\prime}\right)+\left(\varphi\left(x^{\prime}\right)-\varphi\left(w^{\prime}\right)\right)(t-1), & t \in(1,2],\end{cases} \\
& f(t)=\gamma_{2}(t)-\gamma_{1}(t) .
\end{aligned}
$$

Then

$$
f(0)=\varphi\left(z^{\prime}\right)-\varphi\left(x^{\prime}\right)>0, \quad f(2)=\varphi\left(x^{\prime}\right)-\varphi\left(z^{\prime}\right)<0,
$$

and $f$ is continuous on $[0,2]$. Hence there exists $t_{0} \in(0,2)$ such that $f\left(t_{0}\right)=0$. In the case in which $t_{0} \leq 1$, we have

$$
\varphi^{-1}\left(\gamma_{1}\left(t_{0}\right)\right)=\varphi^{-1}\left(\gamma_{2}\left(t_{0}\right)\right) \in\left[x^{\prime}, y^{\prime}\right] \cap\left[z^{\prime}, w^{\prime}\right],
$$

and in the case in which $t_{0}>1$, we have

$$
\varphi^{-1}\left(\gamma_{1}\left(t_{0}\right)\right)=\varphi^{-1}\left(\gamma_{2}\left(t_{0}\right)\right) \in\left[y^{\prime}, z^{\prime}\right] \cap\left[x^{\prime}, w^{\prime}\right] .
$$

This contradicts (7.6) or (7.7). Thus $x^{\prime}, y^{\prime}, z^{\prime}$ and $w^{\prime}$ are not collinear.

The following corollary follows immediately from Lemma 7.5. 
Corollary 7.6. Let $\left(X, d_{X}\right)$ be a metric space that satisfies the $\otimes$-inequalities. Suppose $x, y, z, w \in X$ are four distinct points such that $\{x, y, z, w\}$ is under-distance with respect to $\{y, w\}$. Suppose $x^{\prime}, y^{\prime}, z^{\prime}, w^{\prime} \in \mathbb{R}^{2}$ are points such that

$$
\begin{aligned}
& \left\|x^{\prime}-y^{\prime}\right\|=d_{X}(x, y), \quad\left\|y^{\prime}-z^{\prime}\right\|=d_{X}(y, z), \quad\left\|z^{\prime}-x^{\prime}\right\|=d_{X}(z, x) \\
& \left\|x^{\prime}-w^{\prime}\right\|=d_{X}(x, w), \quad\left\|w^{\prime}-z^{\prime}\right\|=d_{X}(w, z),
\end{aligned}
$$

and $w^{\prime}$ is not on the opposite side of $\overleftrightarrow{x^{\prime} z^{\prime}}$ from $y^{\prime}$. Then

$$
y^{\prime} \in \operatorname{conv}\left(\left\{x^{\prime}, z^{\prime}, w^{\prime}\right\}\right)
$$

or

$$
w^{\prime} \in \operatorname{conv}\left(\left\{x^{\prime}, z^{\prime}, y^{\prime}\right\}\right) .
$$

Moreover, (7.8) and (7.9) do not hold simultaneously.

Proof. It follows from Lemma 7.5 that

$$
\left[x^{\prime}, y^{\prime}\right] \cap\left[z^{\prime}, w^{\prime}\right]=\emptyset, \quad\left[x^{\prime}, w^{\prime}\right] \cap\left[y^{\prime}, z^{\prime}\right]=\emptyset,
$$

and $x^{\prime}, y^{\prime}, z^{\prime}$ and $w^{\prime}$ are not collinear, which clearly implies that (7.8) or (7.9) holds. By (7.10), we have $y^{\prime} \neq w^{\prime}$. Together with the fact that $x^{\prime}, y^{\prime}, z^{\prime}$ and $w^{\prime}$ are not collinear, this implies that (7.8) and (7.9) do not hold simultaneously.

Lemma 7.7. Let $\left(X, d_{X}\right)$ be a metric space that satisfies the $\otimes$-inequalities. Suppose $x, y, z, w \in X$ are four distinct points such that $\{x, y, z, w\}$ is over-distance with respect to $\{y, w\}$. Then $\tilde{\angle} y x z+\tilde{Z} z x w>\pi$ or $\tilde{\angle} y z x+$ $\tilde{\angle} x z w>\pi$.

Proof. Define $x^{\prime}, z^{\prime} \in \mathbb{R}^{2}$ by

$$
x^{\prime}=\left(d_{X}(x, z), 0\right), \quad z^{\prime}=(0,0) .
$$

Suppose $y^{\prime}=\left(y^{(1)}, y^{(2)}\right)$ and $w^{\prime}=\left(w^{(1)}, w^{(2)}\right)$ are the points in $\mathbb{R}^{2}$ such that

$$
\begin{aligned}
& \left\|x^{\prime}-y^{\prime}\right\|=d_{X}(x, y), \quad\left\|y^{\prime}-z^{\prime}\right\|=d_{X}(y, z), \quad y^{(2)} \geq 0 \\
& \left\|x^{\prime}-w^{\prime}\right\|=d_{X}(x, w), \quad\left\|w^{\prime}-z^{\prime}\right\|=d_{X}(w, z), \quad w^{(2)} \leq 0 .
\end{aligned}
$$

Then

$$
\left\|y^{\prime}-w^{\prime}\right\|<d_{X}(y, w)
$$

because $\{x, y, z, w\}$ is over-distance with respect to $\{y, w\}$. It follows that

$$
\left[x^{\prime}, z^{\prime}\right] \cap\left[y^{\prime}, w^{\prime}\right]=\emptyset,
$$

because otherwise Lemma 3.2 would imply that $d_{X}(y, w) \leq\left\|y^{\prime}-w^{\prime}\right\|$. We consider four cases.

CASE 1: $y^{\prime} \notin \overleftrightarrow{x^{\prime} z^{\prime}}$ and $w^{\prime} \notin \overleftrightarrow{x^{\prime} z^{\prime}}$. In this case, (7.11) implies that the region determined by the quadrilateral $\left[x^{\prime}, y^{\prime}\right] \cup\left[y^{\prime}, z^{\prime}\right] \cup\left[z^{\prime}, w^{\prime}\right] \cup\left[w^{\prime}, x^{\prime}\right]$ is not convex, and therefore at least one of the interior angle measures of the quadrilateral is greater than $\pi$. It follows that

$$
\tilde{Z} y x z+\tilde{Z} z x w=\angle y^{\prime} x^{\prime} z^{\prime}+\angle z^{\prime} x^{\prime} w^{\prime}>\pi
$$

or

$$
\tilde{\angle} y z x+\tilde{\angle} x z w=\angle y^{\prime} z^{\prime} x^{\prime}+\angle x^{\prime} z^{\prime} w^{\prime}>\pi .
$$

CASE 2: $y^{\prime} \in \overleftrightarrow{x^{\prime} z^{\prime}}$ and $w^{\prime} \notin \overleftrightarrow{x^{\prime} z^{\prime}}$. In this case, (7.11) implies that one of the following inequalities holds:

$$
y^{(1)}<0, \quad d_{X}(x, z)<y^{(1)} .
$$


If $y^{(1)}<0$, then

$$
\tilde{\angle} y z x+\tilde{\angle} x z w=\angle y^{\prime} z^{\prime} x^{\prime}+\angle x^{\prime} z^{\prime} w^{\prime}=\pi+\angle x^{\prime} z^{\prime} w^{\prime}>\pi .
$$

If $d_{X}(x, z)<y^{(1)}$, then

$$
\tilde{\angle} y x z+\tilde{L} z x w=\angle y^{\prime} x^{\prime} z^{\prime}+\angle z^{\prime} x^{\prime} w^{\prime}=\pi+\angle z^{\prime} x^{\prime} w^{\prime}>\pi .
$$

CASE 3: $y^{\prime} \notin \overleftrightarrow{x^{\prime} z^{\prime}}$ and $w^{\prime} \in \overleftrightarrow{x^{\prime} z^{\prime}}$. In this case, we can prove that $\tilde{\angle} y x z+\tilde{\angle} z x w>\pi$ or $\tilde{\angle} y z x+\tilde{\angle} x z w>\pi$ holds in exactly the same way as in CASE 2.

CASE 4: $y^{\prime} \in \overleftrightarrow{x^{\prime} z^{\prime}}$ and $w^{\prime} \in \overleftrightarrow{x^{\prime} z^{\prime}}$. In this case, (7.11) implies that one of the following inequalities holds:

$$
\max \left\{y^{(1)}, w^{(1)}\right\}<0, \quad d_{X}(x, z)<\min \left\{y^{(1)}, w^{(1)}\right\} .
$$

If $\max \left\{y^{(1)}, w^{(1)}\right\}<0$, then

$$
\tilde{Z} y z x+\tilde{Z} x z w=\angle y^{\prime} z^{\prime} x^{\prime}+\angle x^{\prime} z^{\prime} w^{\prime}=2 \pi>\pi .
$$

If $d_{X}(x, z)<\min \left\{y^{(1)}, w^{(1)}\right\}$, then

$$
\tilde{\angle} y x z+\tilde{\angle} z x w=\angle y^{\prime} x^{\prime} z^{\prime}+\angle z^{\prime} x^{\prime} w^{\prime}=2 \pi>\pi .
$$

The above four cases exhaust all possibilities.

Lemma 7.8. Let $\left(X, d_{X}\right)$ be a metric space that satisfies the $\otimes$-inequalities, and let $x, y, z, w \in X$ be four distinct points such that $\{x, y, z, w\}$ is under-distance with respect to $\{x, w\}$ and $\{y, w\}$. Suppose $x^{\prime}, y^{\prime}, z^{\prime} \in \mathbb{R}^{2}$ are points such that

$$
\left\|x^{\prime}-y^{\prime}\right\|=d_{X}(x, y), \quad\left\|y^{\prime}-z^{\prime}\right\|=d_{X}(y, z), \quad\left\|z^{\prime}-x^{\prime}\right\|=d_{X}(z, x) .
$$

Suppose $w^{\prime} \in \mathbb{R}^{2}$ is a point such that

$$
\left\|y^{\prime}-w^{\prime}\right\|=d_{X}(y, w), \quad\left\|w^{\prime}-z^{\prime}\right\|=d_{X}(w, z),
$$

and $w^{\prime}$ is not on the opposite side of $\overleftrightarrow{y^{\prime} z^{\prime}}$ from $x^{\prime}$. Suppose $w^{\prime \prime} \in \mathbb{R}^{2}$ is a point such that

$$
\left\|x^{\prime}-w^{\prime \prime}\right\|=d_{X}(x, w), \quad\left\|w^{\prime \prime}-z^{\prime}\right\|=d_{X}(w, z),
$$

and $w^{\prime \prime}$ is not on the opposite side of $\overleftrightarrow{x^{\prime} z^{\prime}}$ from $y^{\prime}$. Then

$$
\begin{aligned}
& w^{\prime} \in \operatorname{conv}\left(\left\{x^{\prime}, y^{\prime}, z^{\prime}\right\}\right), \quad w^{\prime \prime} \in \operatorname{conv}\left(\left\{x^{\prime}, y^{\prime}, z^{\prime}\right\}\right), \\
& \operatorname{conv}\left(\left\{y^{\prime}, z^{\prime}, w^{\prime}\right\}\right) \cap \operatorname{conv}\left(\left\{x^{\prime}, z^{\prime}, w^{\prime \prime}\right\}\right)=\left\{z^{\prime}\right\} .
\end{aligned}
$$

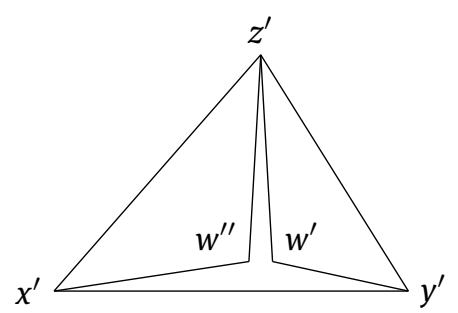

Figure 7.1: The points in $\mathbb{R}^{2}$ appeared in the statement of Lemma 7.8.

Proof. Because $\{x, y, z, w\}$ is under-distance with respect to $\{x, w\}$ and $\{y, w\}$,

$$
d_{X}(x, w)<\left\|x^{\prime}-w^{\prime}\right\|, \quad d_{X}(y, w)<\left\|y^{\prime}-w^{\prime \prime}\right\| .
$$


The first inequality implies that

$$
0<\left\|x^{\prime}-w^{\prime}\right\|-d_{X}(x, w)=\left\|x^{\prime}-w^{\prime}\right\|-\left\|x^{\prime}-w^{\prime \prime}\right\| \leq\left\|w^{\prime}-w^{\prime \prime}\right\|,
$$

which ensures that $w^{\prime} \neq w^{\prime \prime}$. Let $L \subseteq \mathbb{R}^{2}$ be the perpendicular bisector of the line segment $\left[w^{\prime}, w^{\prime \prime}\right]$. Then $x^{\prime}$ is on the same side of $L$ as $w^{\prime \prime}, y^{\prime}$ is on the same side of $L$ as $w^{\prime}$, and $z^{\prime} \in L$ because

$$
\begin{aligned}
& \left\|x^{\prime}-w^{\prime \prime}\right\|=d_{X}(x, w)<\left\|x^{\prime}-w^{\prime}\right\|, \quad\left\|y^{\prime}-w^{\prime}\right\|=d_{X}(y, w)<\left\|y^{\prime}-w^{\prime \prime}\right\|, \\
& \left\|z^{\prime}-w^{\prime}\right\|=d_{X}(z, w)=\left\|z^{\prime}-w^{\prime \prime}\right\| .
\end{aligned}
$$

It follows that

$$
x^{\prime} \notin \operatorname{conv}\left(\left\{y^{\prime}, z^{\prime}, w^{\prime}\right\}\right), \quad y^{\prime} \notin \operatorname{conv}\left(\left\{x^{\prime}, z^{\prime}, w^{\prime \prime}\right\}\right)
$$

and

$$
\operatorname{conv}\left(\left\{y^{\prime}, z^{\prime}, w^{\prime}\right\}\right) \cap \operatorname{conv}\left(\left\{x^{\prime}, z^{\prime}, w^{\prime \prime}\right\}\right)=\left\{z^{\prime}\right\} .
$$

Because $\{x, y, z, w\}$ is under-distance with respect to $\{x, w\}$ and $\{y, w\}$, (7.12) and Corollary 7.6 imply that

$$
w^{\prime} \in \operatorname{conv}\left(\left\{x^{\prime}, y^{\prime}, z^{\prime}\right\}\right), \quad w^{\prime \prime} \in \operatorname{conv}\left(\left\{x^{\prime}, y^{\prime}, z^{\prime}\right\}\right),
$$

which completes the proof.

Corollary 7.9. Let $\left(X, d_{X}\right)$ be a metric space that satisfies the $\otimes$-inequalities, and let $x, y, z, w \in X$ be four distinct points such that $\{x, y, z, w\}$ is under-distance with respect to $\{x, w\}$ and $\{y, w\}$. Suppose $x^{\prime}, y^{\prime}, z^{\prime} \in \mathbb{R}^{2}$ are points such that

$$
\left\|x^{\prime}-y^{\prime}\right\|=d_{X}(x, y), \quad\left\|y^{\prime}-z^{\prime}\right\|=d_{X}(y, z), \quad\left\|z^{\prime}-x^{\prime}\right\|=d_{X}(z, x) .
$$

Then $x^{\prime}, y^{\prime}$ and $z^{\prime}$ are not collinear.

Proof. Choose a point $w^{\prime} \in \mathbb{R}^{2}$ such that

$$
\left\|x^{\prime}-w^{\prime}\right\|=d_{X}(x, w), \quad\left\|w^{\prime}-z^{\prime}\right\|=d_{X}(w, z),
$$

and $w^{\prime}$ is not on the opposite side of $\overleftrightarrow{x^{\prime} z^{\prime}}$ from $y^{\prime}$. Then Lemma 7.8 implies that $w^{\prime} \in \operatorname{conv}\left(\left\{x^{\prime}, y^{\prime}, z^{\prime}\right\}\right)$ because $\{x, y, z, w\}$ is under-distance with respect to $\{x, w\}$ and $\{y, w\}$. Therefore, if $x^{\prime}, y^{\prime}$ and $z^{\prime}$ were collinear, then $x^{\prime}, y^{\prime}, z^{\prime}$ and $w^{\prime}$ would be collinear, contradicting Lemma 7.5 .

Lemma 7.10. Let $\left(X, d_{X}\right)$ be a metric space that satisfies the $\otimes$-inequalities. Suppose $x, y, z, w \in X$ are four distinct points such that $\{x, y, z, w\}$ is over-distance with respect to $\{x, w\}$ and $\{y, w\}$. Then

$$
\pi<\tilde{Z} x z y+\tilde{L} y z w, \quad \pi<\tilde{\angle} x z y+\tilde{Z} x z w .
$$

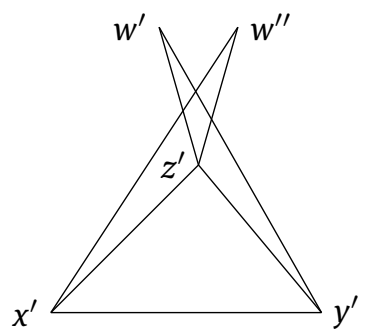

Figure 7.2: The points in $\mathbb{R}^{2}$ appeared in the proof of Lemma 7.10. 
Proof. Choose $x^{\prime}, y^{\prime}, z^{\prime} \in \mathbb{R}^{2}$ such that

$$
\left\|x^{\prime}-y^{\prime}\right\|=d_{X}(x, y), \quad\left\|y^{\prime}-z^{\prime}\right\|=d_{X}(y, z), \quad\left\|z^{\prime}-x^{\prime}\right\|=d_{X}(z, x) .
$$

Suppose $w^{\prime} \in \mathbb{R}^{2}$ is a point such that

$$
\left\|y^{\prime}-w^{\prime}\right\|=d_{X}(y, w), \quad\left\|w^{\prime}-z^{\prime}\right\|=d_{X}(w, z),
$$

and $w^{\prime}$ is not on the same side of $\overleftrightarrow{y^{\prime} z^{\prime}}$ as $x^{\prime}$. Suppose $w^{\prime \prime} \in \mathbb{R}^{2}$ is a point such that

$$
\left\|x^{\prime}-w^{\prime \prime}\right\|=d_{X}(x, w), \quad\left\|w^{\prime \prime}-z^{\prime}\right\|=d_{X}(w, z),
$$

and $w^{\prime \prime}$ is not on the same side of $\overleftrightarrow{x^{\prime} z^{\prime}}$ as $y^{\prime}$. Then because $\{x, y, z, w\}$ is over-distance with respect to $\{x, w\}$ and $\{y, w\}$,

$$
\left\|x^{\prime}-w^{\prime}\right\|<d_{X}(x, w), \quad\left\|y^{\prime}-w^{\prime \prime}\right\|<d_{X}(y, w) .
$$

The first inequality implies that

$$
0<d_{X}(x, w)-\left\|x^{\prime}-w^{\prime}\right\|=\left\|x^{\prime}-w^{\prime \prime}\right\|-\left\|x^{\prime}-w^{\prime}\right\| \leq\left\|w^{\prime \prime}-w^{\prime}\right\|,
$$

which ensures that $w^{\prime} \neq w^{\prime \prime}$. Let $L \subseteq \mathbb{R}^{2}$ be the perpendicular bisector of the line segment $\left[w^{\prime}, w^{\prime \prime}\right]$. Then $x^{\prime}$ is on the same side of $L$ as $w^{\prime}, y^{\prime}$ is on the same side of $L$ as $w^{\prime \prime}$, and $z^{\prime} \in L$ because

$$
\begin{aligned}
& \left\|x^{\prime}-w^{\prime}\right\|<d_{X}(x, w)=\left\|x^{\prime}-w^{\prime \prime}\right\|, \quad\left\|y^{\prime}-w^{\prime \prime}\right\|<d_{X}(y, w)=\left\|y^{\prime}-w^{\prime}\right\|, \\
& \left\|z^{\prime}-w^{\prime}\right\|=d_{X}(z, w)=\left\|z^{\prime}-w^{\prime \prime}\right\| .
\end{aligned}
$$

It follows that

$$
x^{\prime} \notin \operatorname{conv}\left(\left\{y^{\prime}, z^{\prime}, w^{\prime \prime}\right\}\right), \quad y^{\prime} \notin \operatorname{conv}\left(\left\{x^{\prime}, z^{\prime}, w^{\prime}\right\}\right) .
$$

We prove that

$$
\pi<\tilde{Z} x z y+\tilde{Z} y z w
$$

and

$$
\pi<\tilde{\angle} x z y+\tilde{\angle} x z w
$$

by contradiction. If (7.14) were not true, then Lemma 7.7 would imply that

$$
\pi<\tilde{Z} x y z+\tilde{Z} z y w=\angle x^{\prime} y^{\prime} z^{\prime}+\angle z^{\prime} y^{\prime} w^{\prime}
$$

because $\{x, y, z, w\}$ is over-distance with respect to $\{x, w\}$, and therefore Lemma 7.4 and the hypothesis that $w^{\prime}$ is not on the same side of $\overleftrightarrow{y^{\prime} z^{\prime}}$ as $x^{\prime}$ would imply that

$$
y^{\prime} \in \operatorname{conv}\left(\left\{x^{\prime}, z^{\prime}, w^{\prime}\right\}\right),
$$

contradicting (7.13). Similarly, if (7.15) were not true, then we would obtain

$$
x^{\prime} \in \operatorname{conv}\left(\left\{y^{\prime}, z^{\prime}, w^{\prime \prime}\right\}\right),
$$

contradicting (7.13), which completes the proof.

Corollary 7.11. Let $\left(X, d_{X}\right)$ be a metric space that satisfies the $\bigotimes$-inequalities. Suppose $x, y, z, w \in X$ are four distinct points such that $\{x, y, z, w\}$ is over-distance with respect to $\{x, w\}$ and $\{y, w\}$. Then $\{x, y, z, w\}$ is not over-distance with respect to $\{z, w\}$.

Proof. Suppose to the contrary that $\{x, y, z, w\}$ is over-distance with respect to $\{x, w\},\{y, w\}$ and $\{z, w\}$. Then Lemma 7.10 implies that

$$
\pi<\tilde{Z} x y z+\tilde{Z} z y w, \quad \pi<\tilde{Z} x z y+\tilde{Z} y z w,
$$

contradicting the fact that

$$
\begin{aligned}
& (\tilde{\angle} x y z+\tilde{Z} z y w)+(\tilde{\angle} x z y+\tilde{Z} y z w) \\
& \leq(\tilde{Z} x y z+\tilde{Z} x z y+\tilde{Z} z x y)+(\tilde{L} z y w+\tilde{L} y z w+\tilde{Z} z w y)=2 \pi,
\end{aligned}
$$

which proves the corollary. 
Lemma 7.12. Let $\left(X, d_{X}\right)$ be a metric space that satisfies the $\otimes$-inequalities. Suppose $p, x, y, z, w \in X$ are five distinct points such that $\{p, x, y, z\}$ is under-distance with respect to $\{x, y\}$ and $\{y, z\}$, and $\{p, y, z, w\}$ is underdistance with respect to $\{y, z\}$ and $\{z, w\}$. Then

$$
\tilde{Z} x p y+\tilde{Z} y p w<\pi, \quad \tilde{Z} x p z+\tilde{Z} z p w<\pi .
$$

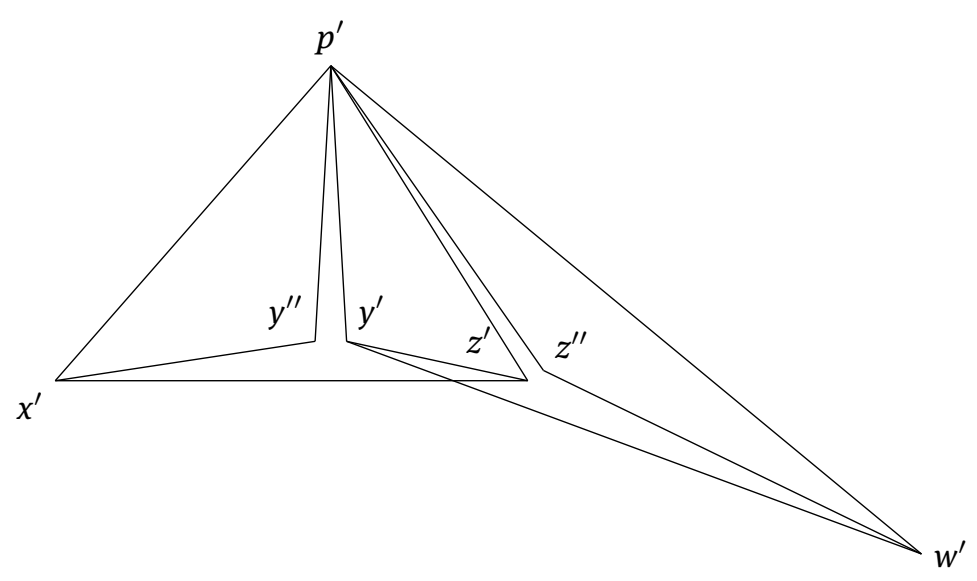

Figure 7.3: The points in $\mathbb{R}^{2}$ appeared in the proof of Lemma 7.12.

Proof. Choose $p^{\prime}, x^{\prime}, z^{\prime} \in \mathbb{R}^{2}$ such that

$$
\left\|p^{\prime}-x^{\prime}\right\|=d_{X}(p, x), \quad\left\|x^{\prime}-z^{\prime}\right\|=d_{X}(x, z), \quad\left\|z^{\prime}-p^{\prime}\right\|=d_{X}(z, p) .
$$

Suppose $y^{\prime} \in \mathbb{R}^{2}$ is a point such that

$$
\left\|p^{\prime}-y^{\prime}\right\|=d_{X}(p, y), \quad\left\|y^{\prime}-z^{\prime}\right\|=d_{X}(y, z),
$$

and $y^{\prime}$ is not on the opposite side of $\overleftrightarrow{p^{\prime} z^{\prime}}$ from $x^{\prime}$. Suppose $y^{\prime \prime} \in \mathbb{R}^{2}$ is a point such that

$$
\left\|p^{\prime}-y^{\prime \prime}\right\|=d_{X}(p, y), \quad\left\|y^{\prime \prime}-x^{\prime}\right\|=d_{X}(y, x),
$$

and $y^{\prime \prime}$ is not on the opposite side of $\overleftrightarrow{p^{\prime} x^{\prime}}$ from $z^{\prime}$. Then because $\{p, x, y, z\}$ is under-distance with respect to $\{x, y\}$ and $\{y, z\}$, Lemma 7.8 implies that

$$
\begin{aligned}
& y^{\prime} \in \operatorname{conv}\left(\left\{p^{\prime}, x^{\prime}, z^{\prime}\right\}\right), \quad y^{\prime \prime} \in \operatorname{conv}\left(\left\{p^{\prime}, x^{\prime}, z^{\prime}\right\}\right), \\
& \operatorname{conv}\left(\left\{z^{\prime}, p^{\prime}, y^{\prime}\right\}\right) \cap \operatorname{conv}\left(\left\{x^{\prime}, p^{\prime}, y^{\prime \prime}\right\}\right)=\left\{p^{\prime}\right\} .
\end{aligned}
$$

Suppose $w^{\prime} \in \mathbb{R}^{2}$ is a point such that

$$
\left\|p^{\prime}-w^{\prime}\right\|=d_{X}(p, w), \quad\left\|w^{\prime}-y^{\prime}\right\|=d_{X}(w, y),
$$

and $w^{\prime}$ is not on the opposite side of $\overleftrightarrow{p^{\prime} y^{\prime}}$ from $z^{\prime}$. Suppose $z^{\prime \prime} \in \mathbb{R}^{2}$ is a point such that

$$
\left\|p^{\prime}-z^{\prime \prime}\right\|=d_{X}(p, z), \quad\left\|z^{\prime \prime}-w^{\prime}\right\|=d_{X}(z, w)
$$

and $z^{\prime \prime}$ is not on the opposite side of $\overleftrightarrow{p^{\prime} w^{\prime}}$ from $y^{\prime}$. Then because $\{p, y, z, w\}$ is under-distance with respect to $\{y, z\}$ and $\{z, w\}$, Lemma 7.8 implies that

$$
\begin{aligned}
& z^{\prime} \in \operatorname{conv}\left(\left\{p^{\prime}, y^{\prime}, w^{\prime}\right\}\right), \quad z^{\prime \prime} \in \operatorname{conv}\left(\left\{p^{\prime}, y^{\prime}, w^{\prime}\right\}\right), \\
& \operatorname{conv}\left(\left\{y^{\prime}, p^{\prime}, z^{\prime}\right\}\right) \cap \operatorname{conv}\left(\left\{w^{\prime}, p^{\prime}, z^{\prime \prime}\right\}\right)=\left\{p^{\prime}\right\} .
\end{aligned}
$$


We define four vectors $\boldsymbol{x}, \boldsymbol{y}, \boldsymbol{z}, \boldsymbol{w} \in \mathbb{R}^{2}$ by

$$
\boldsymbol{x}=x^{\prime}-p^{\prime}, \quad \boldsymbol{y}=y^{\prime}-p^{\prime}, \quad \boldsymbol{z}=z^{\prime}-p^{\prime}, \quad \boldsymbol{w}=w^{\prime}-p^{\prime} .
$$

Then

$$
\begin{array}{ll}
\|\boldsymbol{x}\|=d_{X}(x, p)>0, & \|\boldsymbol{y}\|=d_{X}(y, p)>0, \\
\|\boldsymbol{z}\|=d_{X}(z, p)>0, & \|\boldsymbol{w}\|=d_{X}(w, p)>0 .
\end{array}
$$

Because $\{p, x, y, z\}$ is under-distance with respect to $\{x, y\}$ and $\{y, z\}$, Corollary 7.9 implies that $p^{\prime}, x^{\prime}$ and $z^{\prime}$ are not collinear, and therefore $\boldsymbol{x}$ and $\boldsymbol{z}$ are linearly independent. Because $\{p, y, z, w\}$ is under-distance with respect to $\{y, z\}$ and $\{z, w\}$, Corollary 7.9 implies that $p^{\prime}, y^{\prime}$ and $w^{\prime}$ are not collinear, and therefore $y$ and $\boldsymbol{w}$ are linearly independent. Because $y^{\prime} \notin \overleftrightarrow{p^{\prime} x^{\prime}}$ by (7.16) and (7.17), $\boldsymbol{x}$ and $\boldsymbol{y}$ are linearly independent. Because $z^{\prime} \notin \overleftrightarrow{p^{\prime} w^{\prime}}$ by (7.18) and (7.19), $z$ and $\boldsymbol{w}$ are also linearly independent. By (7.16), there exist $s, t \in[0,1]$ such that $s+t \leq 1$, and $\boldsymbol{y}=\boldsymbol{s} \boldsymbol{x}+t \boldsymbol{z}$. Because

$$
\left\|y^{\prime}-z^{\prime}\right\|=d_{X}(y, z)>0
$$

we have

$$
t<1 \text {. }
$$

By (7.18), there exist $s^{\prime}, t^{\prime} \in[0,1]$ such that $s^{\prime}+t^{\prime} \leq 1$, and

$$
\boldsymbol{z}=s^{\prime} \boldsymbol{y}+t^{\prime} \boldsymbol{w}=s^{\prime}(s \boldsymbol{X}+t \boldsymbol{z})+t^{\prime} \boldsymbol{w} .
$$

Hence

$$
-s s^{\prime} \boldsymbol{x}+\left(1-t s^{\prime}\right) \boldsymbol{z}-t^{\prime} \boldsymbol{w}=\mathbf{0},
$$

where $\mathbf{0}$ denotes the zero vector in $\mathbb{R}^{2}$. By (7.20), we have

$$
1-t s^{\prime}>0 .
$$

Because $\boldsymbol{x}$ and $\boldsymbol{z}$ are linearly independent, it follows from (7.21) and (7.22) that

$$
t^{\prime}>0 \text {. }
$$

Because $\boldsymbol{z}$ and $\boldsymbol{w}$ are linearly independent, it follows from (7.21) and (7.22) that

$$
s s^{\prime}>0 \text {. }
$$

We have

$$
\boldsymbol{w}=-\frac{s s^{\prime}}{t^{\prime}} \boldsymbol{x}+\frac{1-t s^{\prime}}{t^{\prime}} \boldsymbol{z}, \quad \frac{1-t s^{\prime}}{t^{\prime}}>0
$$

by (7.21), (7.22) and (7.23), and therefore $\boldsymbol{x}$ and $\boldsymbol{w}$ are linearly independent, which implies in particular that

$$
\angle x^{\prime} p^{\prime} w^{\prime}<\pi
$$

We also have

$$
\boldsymbol{z}=\frac{s s^{\prime}}{1-t s^{\prime}} \boldsymbol{x}+\frac{t^{\prime}}{1-t s^{\prime}} \boldsymbol{w}, \quad \frac{s s^{\prime}}{1-t s^{\prime}}>0, \quad \frac{t^{\prime}}{1-t s^{\prime}}>0
$$

by (7.21), (7.22), (7.23) and (7.24), and therefore the ray from $p^{\prime}$ through $z^{\prime}$ is between that from $p^{\prime}$ through $x^{\prime}$ and that from $p^{\prime}$ through $w^{\prime}$. Hence

$$
\angle x^{\prime} p^{\prime} z^{\prime}+\angle z^{\prime} p^{\prime} w^{\prime}=\angle x^{\prime} p^{\prime} w^{\prime}
$$

Because $\{p, y, z, w\}$ is under-distance with respect to $\{z, w\}$, we have

$$
\left\|z^{\prime \prime}-p^{\prime}\right\|=\left\|z^{\prime}-p^{\prime}\right\|=d_{X}(z, p)>0, \quad\left\|z^{\prime \prime}-w^{\prime}\right\|=d_{X}(z, w)<\left\|z^{\prime}-w^{\prime}\right\|,
$$


and therefore Lemma 7.3 implies that

$$
\angle z^{\prime \prime} p^{\prime} w^{\prime}<\angle z^{\prime} p^{\prime} w^{\prime}
$$

Combining (7.25), (7.26) and (7.27) yields

$$
\begin{aligned}
\tilde{\angle} x p z+\tilde{\angle} z p w & =\angle x^{\prime} p^{\prime} z^{\prime}+\angle z^{\prime \prime} p^{\prime} w^{\prime} \\
& <\angle x^{\prime} p^{\prime} z^{\prime}+\angle z^{\prime} p^{\prime} w^{\prime} \\
& =\angle x^{\prime} p^{\prime} w^{\prime}<\pi .
\end{aligned}
$$

Clearly the inequality

$$
\tilde{Z} x p y+\tilde{\angle} y p w<\pi
$$

is proved in the same way, which completes the proof.

The following corollary follows from Lemma 7.10 and Lemma 7.12, which will play an important role when we prove that the validity of the $\otimes$-inequalities implies the $G_{9}^{(5)}(0)$ condition in Section 9.

Corollary 7.13. Let $X$ be a metric space that satisfies the $\otimes$-inequalities. Suppose $p, x_{1}, x_{2}, x_{3}, x_{4} \in X$ are five distinct points such that $\left\{p, x_{1}, x_{2}, x_{3}\right\}$ is under-distance with respect to $\left\{x_{1}, x_{2}\right\}$ and $\left\{x_{2}, x_{3}\right\}$, and $\left\{p, x_{3}, x_{4}, x_{1}\right\}$ is over-distance with respect to $\left\{x_{3}, x_{4}\right\}$ and $\left\{x_{4}, x_{1}\right\}$. Assume that neither $\left\{p, x_{2}, x_{3}, x_{4}\right\}$ nor $\left\{p, x_{4}, x_{1}, x_{2}\right\}$ admits an isometric embedding into $\mathbb{R}^{3}$. Then $\left\{p, x_{2}, x_{3}, x_{4}\right\}$ is over-distance with respect to $\left\{x_{2}, x_{3}\right\}$ or $\left\{x_{3}, x_{4}\right\}$, and $\left\{p, x_{4}, x_{1}, x_{2}\right\}$ is over-distance with respect to $\left\{x_{4}, x_{1}\right\}$ or $\left\{x_{1}, x_{2}\right\}$.

Proof. Suppose to the contrary that $\left\{p, x_{2}, x_{3}, x_{4}\right\}$ is neither over-distance with respect to $\left\{x_{2}, x_{3}\right\}$ nor $\left\{x_{3}, x_{4}\right\}$, or $\left\{p, x_{4}, x_{1}, x_{2}\right\}$ is neither over-distance with respect to $\left\{x_{4}, x_{1}\right\}$ nor $\left\{x_{1}, x_{2}\right\}$. We may assume without loss of generality that $\left\{p, x_{2}, x_{3}, x_{4}\right\}$ is neither over-distance with respect to $\left\{x_{2}, x_{3}\right\}$ nor $\left\{x_{3}, x_{4}\right\}$. Then Proposition 7.2 implies that $\left\{p, x_{2}, x_{3}, x_{4}\right\}$ is under-distance with respect to $\left\{x_{2}, x_{3}\right\}$ and $\left\{x_{3}, x_{4}\right\}$ because $\left\{p, x_{2}, x_{3}, x_{4}\right\}$ does not admit an isometric embedding into $\mathbb{R}^{3}$. Combining this with the hypothesis that $\left\{p, x_{1}, x_{2}, x_{3}\right\}$ is under-distance with respect to $\left\{x_{1}, x_{2}\right\}$ and $\left\{x_{2}, x_{3}\right\}$, Lemma 7.12 implies that

$$
\tilde{\angle} x_{1} p x_{3}+\tilde{\angle} x_{3} p x_{4}<\pi \text {. }
$$

On the other hand, because $\left\{p, x_{3}, x_{4}, x_{1}\right\}$ is over-distance with respect to $\left\{x_{3}, x_{4}\right\}$ and $\left\{x_{4}, x_{1}\right\}$, Lemma 7.10 implies that

$$
\pi<\tilde{L} x_{1} p x_{3}+\tilde{L} x_{3} p x_{4},
$$

contradicting (7.28).

We define some notations, which will be used several times in the next two sections.

Let $\left(X, d_{X}\right)$ be a metric space, and let $x, y, z, w \in X$ be four distinct points. Choose points $x_{1}, y_{1}, z_{1}, x_{2}, z_{2}, w_{2}, x_{3}, w_{3}, y_{3} \in \mathbb{R}^{2}$ such that

$$
\begin{aligned}
& \left\|x_{1}-y_{1}\right\|=d_{X}(x, y), \quad\left\|y_{1}-z_{1}\right\|=d_{X}(y, z), \quad\left\|z_{1}-x_{1}\right\|=d_{X}(z, x), \\
& \left\|x_{2}-z_{2}\right\|=d_{X}(x, z), \quad\left\|z_{2}-w_{2}\right\|=d_{X}(z, w), \quad\left\|w_{2}-x_{2}\right\|=d_{X}(w, x), \\
& \left\|x_{3}-w_{3}\right\|=d_{X}(x, w), \quad\left\|w_{3}-y_{3}\right\|=d_{X}(w, y), \quad\left\|y_{3}-x_{3}\right\|=d_{X}(y, x) .
\end{aligned}
$$

Equip the subsets

$$
T_{1}=\operatorname{conv}\left(\left\{x_{1}, y_{1}, z_{1}\right\}\right), \quad T_{2}=\operatorname{conv}\left(\left\{x_{2}, z_{2}, w_{2}\right\}\right), \quad T_{3}=\operatorname{conv}\left(\left\{x_{3}, w_{3}, y_{3}\right\}\right)
$$

of $\mathbb{R}^{2}$ with the induced metrics, and regard them as disjoint metric spaces. We denote by $D(x ; y, z, w)$ the piecewise Euclidean metric simplicial complex constructed from $T_{1}, T_{2}$ and $T_{3}$ by identifying $\left[x_{1}, z_{1}\right] \subseteq T_{1}$ with $\left[x_{2}, z_{2}\right] \subseteq T_{2},\left[x_{2}, w_{2}\right] \subseteq T_{2}$ with $\left[x_{3}, w_{3}\right] \subseteq T_{3}$, and $\left[x_{3}, y_{3}\right] \subseteq T_{3}$ with $\left[x_{1}, y_{1}\right] \subseteq T_{1}$. We denote the images of $T_{1}, T_{2}$ and $T_{3}$ under the natural inclusions into $D(x ; y, z, w)$ by $T_{D(x ; y, z, w)}(x, y, z), T_{D(x ; y, z, w)}(x, z, w)$ and $T_{D(x ; y, z, w)}(x, w, y)$, respectively. When there is no risk of confusion, we abbreviate these notations by 
$T(x, y, z), T(x, z, w)$ and $T(x, w, y)$, respectively. The map from $\{x, y, z, w\}$ to $D(x ; y, z, w)$ sending $x, y, z$ and $w$ to the points in $D(x ; y, z, w)$ represented by $x_{1}, y_{1}, z_{1} \in T_{1}$ and $w_{2} \in T_{2}$, respectively is called the natural inclusion of $\{x, y, z, w\}$ into $D(x ; y, z, w)$. Clearly, up to isometry, $D(x ; y, z, w), T(x, y, z), T(x, z, w)$, $T(x, w, y)$ and the natural inclusion of $\{x, y, z, w\}$ into $D(x ; y, z, w)$ are independent of the choice of the points $x_{1}, y_{1}, z_{1}, x_{2}, z_{2}, w_{2}, x_{3}, w_{3}, y_{3} \in \mathbb{R}^{2}$.

Lemma 7.14. Let $\left(X, d_{X}\right)$ be a metric space that satisfies the $\otimes$-inequalities. Suppose $x, y, z, w \in X$ are four distinct points such that $\{x, y, z, w\}$ is over-distance with respect to $\{y, w\}$, and

$$
\pi<\tilde{\angle} y x z+\tilde{\angle} z x w .
$$

Then $D(x ; y, z, w)$ is a CAT(0) space, and for each $i \in\{1,2,3\}$, the natural inclusion of a (possibly degenerate) triangular region $T_{i} \subseteq \mathbb{R}^{2}$ as in (7.30) into $D(x ; y, z, w)$ is an isometric embedding. In particular, $T(x, y, z), T(x, z, w)$ and $T(x, w, y)$ are closed convex subsets of $D(x ; y, z, w)$. Moreover, the natural inclusion of $\{x, y, z, w\}$ into $D(x ; y, z, w)$ is an isometric embedding.

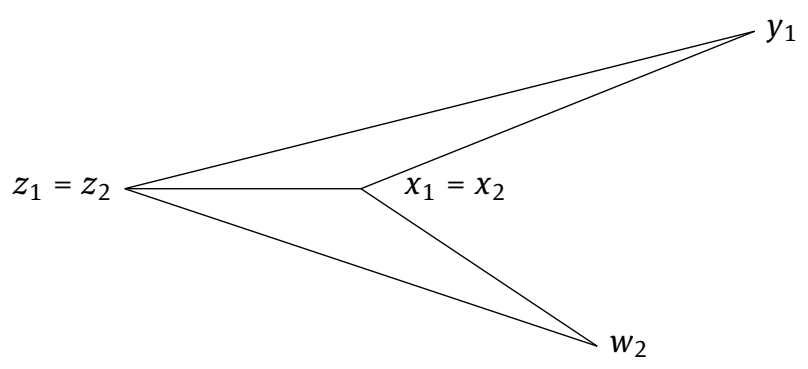

Figure 7.4: Points in $\mathbb{R}^{2}$ appeared in the proof of Lemma 7.14.

Proof. Suppose $x_{1}, y_{1}, z_{1}, x_{2}, z_{2}, w_{2}, x_{3}, w_{3}, y_{3} \in \mathbb{R}^{2}$ are points satisfying (7.29). By transforming $x_{2}, z_{2}$ and $w_{2}$ if necessary, we may assume that $x_{1}=x_{2}, z_{1}=z_{2}$, and $w_{2}$ is not on the same side of $\overleftrightarrow{x_{1} z_{1}}$ as $y_{1}$, as shown in Figure 7.4. By (7.31),

$$
\pi<\angle y_{1} x_{1} z_{1}+\angle z_{2} x_{2} w_{2}=\angle y_{1} x_{1} z_{1}+\angle z_{1} x_{1} w_{2},
$$

which implies that

$$
\angle y_{1} x_{1} z_{1}+\angle z_{1} x_{1} w_{2}+\angle w_{2} x_{1} y_{1}=2 \pi .
$$

Because $\{x, y, z, w\}$ is over-distance with respect to $\{y, w\}$,

$$
\left\|y_{1}-w_{2}\right\|<d_{X}(y, w) .
$$

Hence we have

$$
\begin{aligned}
& \left\|x_{1}-y_{1}\right\|=d_{X}(x, y)=\left\|x_{3}-y_{3}\right\|, \quad\left\|x_{1}-w_{2}\right\|=d_{X}(x, w)=\left\|x_{3}-w_{3}\right\|, \\
& \left\|y_{1}-w_{2}\right\|<d_{X}(y, w)=\left\|y_{3}-w_{3}\right\|,
\end{aligned}
$$

and therefore Lemma 7.3 implies that

$$
\angle w_{2} x_{1} y_{1}<\angle w_{3} x_{3} y_{3}
$$

Combining this with (7.33) yields

$$
2 \pi<\angle y_{1} x_{1} z_{1}+\angle z_{1} x_{1} w_{2}+\angle w_{3} x_{3} y_{3}=\angle y_{1} x_{1} z_{1}+\angle z_{2} x_{2} w_{2}+\angle w_{3} x_{3} y_{3} .
$$

For each $i \in\{1,2,3\}$, let $T_{i}$ be the (possibly degenerate) triangular region defined by (7.30). As we mentioned in Example 2.2, (7.34) ensures that $D(x ; y, z, w)$ is a CAT(0) space, and that for each $i \in\{1,2,3\}$, 
the natural inclusion of $T_{i}$ into $D(x ; y, z, w)$ is an isometric embedding. In particular, the natural inclusion $\varphi:\{x, y, z, w\} \rightarrow D(x ; y, z, w)$ is an isometric embedding because for any $a, b \in\{x, y, z, w\}$, both $\varphi(a)$ and $\varphi(b)$ are represented by elements of $T_{i}$ for some $i \in\{1,2,3\}$.

Remark 7.15. Suppose $X$ is a metric space that satisfies the $\otimes$-inequalities, and $x, y, z, w \in X$ are four distinct points such that $\{x, y, z, w\}$ is over-distance with respect to $\{y, w\}$. Then Lemma 7.7 implies that

$$
\pi<\tilde{Z} y x z+\tilde{Z} z x w,
$$

or

$$
\pi<\tilde{L} y z x+\tilde{L} x z w .
$$

Thus renaming the points if necessary, the points $x, y, z$ and $w$ always satisfy the condition (7.31), and therefore $D(x ; y, z, w)$ becomes a CAT( 0$)$ space, and the natural inclusion of $\{x, y, z, w\}$ into $D(x ; y, z, w)$ becomes an isometric embedding by Lemma 7.14 .

\section{The $G_{7}^{(5)}(0)$ condition}

In this section, we prove that the validity of the $\otimes$-inequalities implies the $G_{7}^{(5)}(0)$ condition. We start with the following three simple facts. All of them hold clearly, so we omit their proofs.

Lemma 8.1 (cf. [4, p.25, 2.16(1)]). Let $x, y, z, w \in \mathbb{R}^{2}$. If $w \in \operatorname{conv}(\{x, y, z\})$, then

$$
\|x-w\|+\|w-y\| \leq\|x-z\|+\|z-y\| .
$$

If in addition $x, y, z$ and $w$ are distinct, and $\angle y x w<\angle y x z$, then strict inequality holds in (8.1).

Lemma 8.2. Let $o \in \mathbb{R}^{2}$. Suppose $x, y, z \in \mathbb{R}^{2} \backslash\{0\}$ are points such that $y$ and $z$ are not on opposite sides of $\overleftrightarrow{o x}$, and $\angle x o y \leq \angle x o z$. Then $x$ and $z$ are not on the same side of $\overleftrightarrow{o y}$, and $\angle x o z=\angle x o y+\angle y o z$.

Lemma 8.3. Suppose $o, x, y \in \mathbb{R}^{2}$ are points that are not collinear. Suppose $p, q \in \mathbb{R}^{2}$ are points such that neither $p$ nor $q$ is on the same side of $\overleftrightarrow{o y}$ as $x$, and $p$ is not on the same side of $\overleftrightarrow{o x}$ as $q$. Then $\angle p x q=\angle p x o+\angle o x q$.

We use these facts to prove the following lemma, which will play a key role to prove that the validity of the $\triangle$-inequalities implies the $G_{7}^{(5)}(0)$ condition.

Lemma 8.4. Suppose $x, y, z, w \in \mathbb{R}^{2}$ are four distinct points such that $w \in \operatorname{conv}(\{x, y, z\})$. Suppose $x^{\prime}, y^{\prime}, z^{\prime}, w^{\prime} \in \mathbb{R}^{2}$ are points such that

$$
\begin{aligned}
& \left\|x^{\prime}-z^{\prime}\right\|=\|x-z\|, \quad\left\|z^{\prime}-y^{\prime}\right\|=\|z-y\|, \quad\left\|x^{\prime}-w^{\prime}\right\|=\|x-w\|, \quad\left\|w^{\prime}-y^{\prime}\right\|=\|w-y\| . \\
& \text { If }\left\|z^{\prime}-w^{\prime}\right\| \leq\|z-w\| \text {, then }\left\|x^{\prime}-y^{\prime}\right\| \leq\|x-y\| .
\end{aligned}
$$

Proof. Suppose $x, y, z, w \in \mathbb{R}^{2}$ are four distinct points such that $w \in \operatorname{conv}(\{x, y, z\})$, and $x^{\prime}, y^{\prime}, z^{\prime}, w^{\prime} \in \mathbb{R}^{2}$ are points satisfying (8.2). To prove the lemma by contrapositive, we assume that

$$
\|x-y\|<\left\|x^{\prime}-y^{\prime}\right\| .
$$

Then $x^{\prime} \neq y^{\prime}$ because $0<\|x-y\|$. Choose a point $\bar{z} \in \mathbb{R}^{2}$ such that

$$
\left\|x^{\prime}-\bar{z}\right\|=\left\|x^{\prime}-z^{\prime}\right\|, \quad\left\|\bar{z}-y^{\prime}\right\|=\left\|z^{\prime}-y^{\prime}\right\|,
$$

and $\bar{z}$ is not on the opposite side of $\overleftrightarrow{x^{\prime} y^{\prime}}$ from $w^{\prime}$. If $z^{\prime}$ and $w^{\prime}$ are not on opposite sides of $\overleftrightarrow{x^{\prime} y^{\prime}}$, we may choose $\bar{z}=z^{\prime}$. Otherwise, $\bar{z}$ is the point obtained by reflecting $z^{\prime}$ orthogonally across $\overleftrightarrow{x^{\prime} y^{\prime}}$. Clearly,

$$
\left\|\bar{z}-w^{\prime}\right\| \leq\left\|z^{\prime}-w^{\prime}\right\| .
$$


Because $w \in \operatorname{conv}(\{x, y, z\})$, Lemma 8.1 implies that

$$
\left\|x^{\prime}-w^{\prime}\right\|+\left\|w^{\prime}-y^{\prime}\right\|=\|x-w\|+\|w-y\| \leq\|x-z\|+\|z-y\|=\left\|x^{\prime}-\bar{z}\right\|+\left\|\bar{z}-y^{\prime}\right\| .
$$

If $\angle y^{\prime} x^{\prime} \bar{z}$ were less than $\angle y^{\prime} x^{\prime} w^{\prime}$, and $\angle x^{\prime} y^{\prime} \bar{z}$ were less than $\angle x^{\prime} y^{\prime} w^{\prime}$, then $\bar{z}$ would lie in $\operatorname{conv}\left(\left\{x^{\prime}, y^{\prime}, w^{\prime}\right\}\right)$, and therefore Lemma 8.1 would imply that

$$
\left\|x^{\prime}-\bar{z}\right\|+\left\|\bar{z}-y^{\prime}\right\|<\left\|x^{\prime}-w^{\prime}\right\|+\left\|w^{\prime}-y^{\prime}\right\|,
$$

contradicting (8.6). Thus $\angle y^{\prime} x^{\prime} w^{\prime} \leq \angle y^{\prime} x^{\prime} \bar{z}$ or $\angle x^{\prime} y^{\prime} w^{\prime} \leq \angle x^{\prime} y^{\prime} \bar{z}$. We may assume without loss of generality that $\angle y^{\prime} x^{\prime} w^{\prime} \leq \angle y^{\prime} x^{\prime} \bar{z}$. Then Lemma 8.2 implies that

$$
\angle y^{\prime} x^{\prime} \bar{z}=\angle y^{\prime} x^{\prime} w^{\prime}+\angle w^{\prime} x^{\prime} \bar{z}
$$

because $\bar{z}$ is not on the opposite side of $\overleftrightarrow{x^{\prime} y^{\prime}}$ from $w^{\prime}$ by definition. We consider two cases.

CASE 1: $x, y$ and $w$ are not collinear. Suppose $\tilde{y} \in \mathbb{R}^{2}$ is the point such that

$$
\|\tilde{y}-w\|=\|y-w\|, \quad \angle x w \tilde{y}=\angle x^{\prime} w^{\prime} y^{\prime},
$$

and $\tilde{y}$ is not on the opposite side of $\overleftrightarrow{w x}$ from $y$, as shown in FIGURE 8.1. Then the triangle with vertices $x, \tilde{y}$
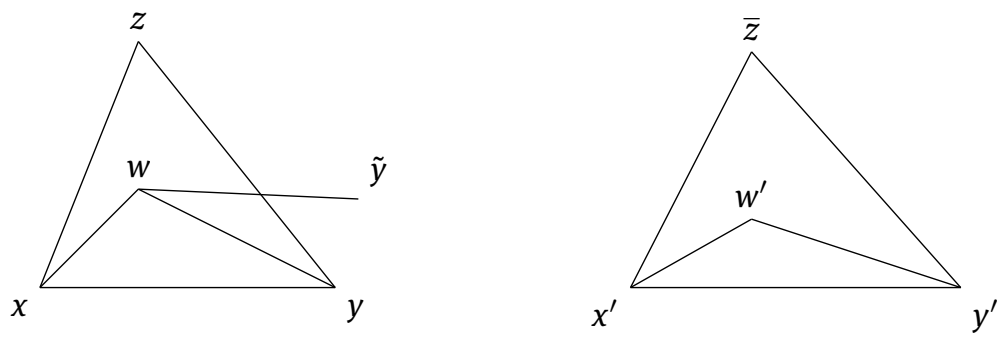

Figure 8.1: Proof of Lemma 8.4.

and $w$ is congruent to that with vertices $x^{\prime}, y^{\prime}$ and $w^{\prime}$. Hence

$$
\|x-\tilde{y}\|=\left\|x^{\prime}-y^{\prime}\right\|, \quad \angle \tilde{y} x w=\angle y^{\prime} x^{\prime} w^{\prime} .
$$

Because $\|x-w\|=\left\|x^{\prime}-w^{\prime}\right\|$ and $\|y-w\|=\left\|y^{\prime}-w^{\prime}\right\|$, (8.3) and Lemma 7.3 imply that

$$
\angle x w y<\angle x^{\prime} w^{\prime} y^{\prime}=\angle x w \tilde{y} .
$$

Because $\tilde{y}$ is not on the opposite side of $\overleftrightarrow{w x}$ from $y$ by definition, (8.9) and Lemma 8.2 imply that

$$
\angle x w y+\angle y w \tilde{y}=\angle x w \tilde{y} \leq \pi .
$$

Because $w \in \operatorname{conv}(\{x, y, z\})$, Lemma 7.4 implies that

$$
\pi \leq \angle x w y+\angle y w z .
$$

Combining this with (8.10) yields $\angle y w \tilde{y} \leq \angle y w z$. Furthermore, $\tilde{y}$ and $z$ are not on opposite sides of $\overleftrightarrow{w y}$ because neither $\tilde{y}$ nor $z$ is on the same side of $\overleftrightarrow{w y}$ as $x$ by Lemma 8.2 and Lemma 7.4, respectively, and $x \notin \overleftrightarrow{w y}$ by the assumption of CASE 1. Therefore, Lemma 8.2 implies that

$$
\angle y w z=\angle y w \tilde{y}+\angle \tilde{y} w z .
$$

Because $0<\angle y w \tilde{y}$ by (8.9) and (8.10), it follows that

$$
\angle \tilde{y} w z<\angle y w z \text {. }
$$


Because $\|y-w\|=\|\tilde{y}-w\|$ by definition of $\tilde{y}$, this implies that

$$
\|\tilde{y}-z\|<\|y-z\|=\left\|y^{\prime}-\bar{z}\right\|
$$

by Lemma 7.3. Because $\|x-z\|=\left\|x^{\prime}-\bar{z}\right\|$, and $\|x-\tilde{y}\|=\left\|x^{\prime}-y^{\prime}\right\|$ by (8.8), it follows from (8.11) and Lemma 7.3 that

$$
\angle \tilde{y} x z<\angle y^{\prime} x^{\prime} \bar{z}
$$

As we mentioned above, neither $\tilde{y}$ nor $z$ is on the same side of $\overleftrightarrow{w y}$ as $x$. Furthermore, $\tilde{y}$ and $z$ are not on the same side of $\overleftrightarrow{w x}$ because $\tilde{y}$ is not on the opposite side of $\overleftrightarrow{w x}$ from $y$ by definition of $\tilde{y}, z$ is not on the same side of $\overleftrightarrow{w x}$ as $y$ by Lemma 7.4, and $y \notin \overleftrightarrow{w x}$ by the assumption of CASE 1 . Therefore, Lemma 8.3 implies that

$$
\angle \tilde{y} x z=\angle \tilde{y} x w+\angle w x z .
$$

By (8.7), (8.8), (8.12) and (8.13),

$$
\begin{aligned}
\angle w x z & =\angle \tilde{y} x z-\angle \tilde{y} x w \\
& <\angle y^{\prime} x^{\prime} \bar{z}-\angle \tilde{y} x w \\
& =\angle y^{\prime} x^{\prime} \bar{z}-\angle y^{\prime} x^{\prime} w^{\prime} \\
& =\angle w^{\prime} x^{\prime} \bar{z} .
\end{aligned}
$$

Hence Lemma 7.3 implies that

$$
\|z-w\|<\left\|\bar{z}-w^{\prime}\right\|
$$

because $\|z-x\|=\left\|\bar{z}-x^{\prime}\right\|$ and $\|w-x\|=\left\|w^{\prime}-x^{\prime}\right\|$. Combining this with (8.5) yields

$$
\|z-w\|<\left\|z^{\prime}-w^{\prime}\right\| \text {. }
$$

CASE 2: $x, y$ and $w$ are collinear. In this case, $w \in \overleftrightarrow{x y} \backslash[x, y]$, because otherwise we would have

$$
\left\|x^{\prime}-y^{\prime}\right\| \leq\left\|x^{\prime}-w^{\prime}\right\|+\left\|w^{\prime}-y^{\prime}\right\|=\|x-w\|+\|w-y\|=\|x-y\|,
$$

contradicting (8.3). Because $w \in \operatorname{conv}(\{x, y, z\})$, it follows that

$$
w \in[x, z] \cap[y, z],
$$

which implies in particular that

$$
\|z-w\|=|\|x-z\|-\|x-w\||=\left|\left\|x^{\prime}-z^{\prime}\right\|-\left\|x^{\prime}-w^{\prime}\right\|\right| \leq\left\|z^{\prime}-w^{\prime}\right\| .
$$

To prove that equality does not hold in the inequality in (8.15), suppose to the contrary that $\|z-w\|=\left\|z^{\prime}-w^{\prime}\right\|$. Then (8.14) implies that

$$
\begin{aligned}
& \left\|x^{\prime}-z^{\prime}\right\|=\|x-z\|=\|x-w\|+\|w-z\|=\left\|x^{\prime}-w^{\prime}\right\|+\left\|w^{\prime}-z^{\prime}\right\|, \\
& \left\|y^{\prime}-z^{\prime}\right\|=\|y-z\|=\|y-w\|+\|w-z\|=\left\|y^{\prime}-w^{\prime}\right\|+\left\|w^{\prime}-z^{\prime}\right\|,
\end{aligned}
$$

and thus

$$
w^{\prime} \in\left[x^{\prime}, z^{\prime}\right] \cap\left[y^{\prime}, z^{\prime}\right]
$$

On the other hand, we have $x^{\prime} \notin\left[y^{\prime}, z^{\prime}\right]$ and $y^{\prime} \notin\left[x^{\prime}, z^{\prime}\right]$, because otherwise we would have

$$
\left\|x^{\prime}-y^{\prime}\right\|=\left|\left\|x^{\prime}-z^{\prime}\right\|-\left\|y^{\prime}-z^{\prime}\right\|\right|=|\|x-z\|-\|y-z\|| \leq\|x-y\|,
$$

contradicting (8.3). Hence

$$
\left[x^{\prime}, z^{\prime}\right] \cap\left[y^{\prime}, z^{\prime}\right]=\left\{z^{\prime}\right\} .
$$

Combining this with (8.16) yields $z^{\prime}=w^{\prime}$. Therefore,

$$
\begin{aligned}
& \|x-z\|=\left\|x^{\prime}-z^{\prime}\right\|=\left\|x^{\prime}-w^{\prime}\right\|=\|x-w\|, \\
& \|y-z\|=\left\|y^{\prime}-z^{\prime}\right\|=\left\|y^{\prime}-w^{\prime}\right\|=\|y-w\| .
\end{aligned}
$$

Because $w \in \operatorname{conv}(\{x, y, z\})$, these equalities imply that $z=w$, contradicting the hypothesis that $z \neq w$. Thus equality does not hold in the inequality in (8.15), which completes the proof of the lemma. 
We are ready to prove the following proposition.

Proposition 8.5. If a metric space $X$ satisfies the $\otimes$-inequalities, then $X$ satisfies the $G_{7}^{(5)}(0)$ condition.

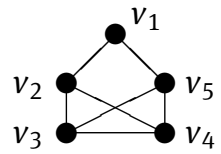

Figure 8.2

Proof. Let $\left(X, d_{X}\right)$ be a metric space that satisfies the $\otimes$-inequalities. Let $V$ and $E$ be the vertex set and the edge set of $G_{7}^{(5)}(0)$, respectively. We set

$$
\begin{aligned}
& V=\left\{v_{1}, v_{2}, v_{3}, v_{4}, v_{5}\right\}, \\
& E=\left\{\left\{v_{1}, v_{2}\right\},\left\{v_{2}, v_{3}\right\},\left\{v_{3}, v_{4}\right\},\left\{v_{4}, v_{5}\right\},\left\{v_{5}, v_{1}\right\},\left\{v_{2}, v_{4}\right\},\left\{v_{3}, v_{5}\right\}\right\},
\end{aligned}
$$

as shown in Figure 8.2. Fix a map $f: V \rightarrow X$, and set

$$
d_{i j}=d_{X}\left(f\left(v_{i}\right), f\left(v_{j}\right)\right)
$$

for any $i, j \in\{1,2,3,4,5\}$. By Theorem 1.7, if $d_{i j}=0$ for some $i, j \in\{1,2,3,4,5\}$ with $i \neq j$, then there exist a CAT(0) space $\left(Y_{0}, d_{Y_{0}}\right)$ and a map $g_{0}: V \rightarrow Y_{0}$ such that $d_{Y_{0}}\left(g_{0}\left(v_{i}\right), g_{0}\left(v_{j}\right)\right)=d_{i j}$ for any $i, j \in\{1,2,3,4,5\}$. Therefore, we assume that $d_{i j}>0$ for any $i, j \in\{1,2,3,4,5\}$ with $i \neq j$. Choose $p_{1}, p_{2}, p_{5} \in \mathbb{R}^{2}$ such that

$$
\left\|p_{1}-p_{2}\right\|=d_{12}, \quad\left\|p_{2}-p_{5}\right\|=d_{25}, \quad\left\|p_{5}-p_{1}\right\|=d_{51} .
$$

Equip the subset $P=\operatorname{conv}\left(\left\{p_{1}, p_{2}, p_{5}\right\}\right)$ of $\mathbb{R}^{2}$ with the induced metric, and regard it as a metric space in its own right. We consider three cases.

CASE 1: The subset $\left\{f\left(v_{2}\right), f\left(v_{3}\right), f\left(v_{4}\right), f\left(v_{5}\right)\right\}$ of $X$ admits an isometric embedding into $\mathbb{R}^{3}$. Let $\varphi$ : $\left\{f\left(v_{2}\right), f\left(v_{3}\right), f\left(v_{4}\right), f\left(v_{5}\right)\right\} \rightarrow \mathbb{R}^{3}$ be an isometric embedding. Define $\left(Y_{1}, d_{Y_{1}}\right)$ to be the metric space obtained by gluing $P$ and $\mathbb{R}^{3}$ by identifying $\left[p_{2}, p_{5}\right] \subseteq P$ with $\left[\varphi\left(f\left(v_{2}\right)\right), \varphi\left(f\left(v_{5}\right)\right)\right] \subseteq \mathbb{R}^{3}$. Then $\left(Y_{1}, d_{Y_{1}}\right)$ is a CAT(0) space by Reshetnyak's gluing theorem. Define a map $g_{1}: V \rightarrow Y_{1}$ by sending $v_{i}$ to the point in $Y_{1}$ represented by $\varphi\left(f\left(v_{i}\right)\right) \in \mathbb{R}^{3}$ for each $i \in\{2,3,4,5\}$, and $v_{1}$ to the point in $Y_{1}$ represented by $p_{1} \in P$. Then

$$
\begin{aligned}
& d_{Y_{1}}\left(g_{1}\left(v_{1}\right), g_{1}\left(v_{i}\right)\right)=\left\|p_{1}-p_{i}\right\|=d_{1 i}, \\
& d_{Y_{1}}\left(g_{1}\left(v_{j}\right), g_{1}\left(v_{k}\right)\right)=\left\|\varphi\left(f\left(v_{j}\right)\right)-\varphi\left(f\left(v_{k}\right)\right)\right\|=d_{j k}
\end{aligned}
$$

for any $i \in\{2,5\}$ and any $j, k \in\{2,3,4,5\}$. It is clear from the definitions of $Y_{1}$ and $g_{1}$ that

$$
\begin{aligned}
& {\left[g_{1}\left(v_{2}\right), g_{1}\left(v_{5}\right)\right] \cap\left[g_{1}\left(v_{1}\right), g_{1}\left(v_{i}\right)\right] \neq \emptyset,} \\
& \operatorname{conv}\left(\left\{g_{1}\left(v_{1}\right), g_{1}\left(v_{2}\right), g_{1}\left(v_{5}\right)\right\}\right) \cap \operatorname{conv}\left(\left\{g_{1}\left(v_{i}\right), g_{1}\left(v_{2}\right), g_{1}\left(v_{5}\right)\right\}\right)=\left[g_{1}\left(v_{2}\right), g_{1}\left(v_{5}\right)\right]
\end{aligned}
$$

for each $i \in\{3,4\}$, and $\operatorname{conv}\left(\left\{g_{1}\left(v_{j}\right), g_{1}\left(v_{2}\right), g_{1}\left(v_{5}\right)\right\}\right)$ is isometric to a convex subset of the Euclidean plane for each $j \in\{1,3,4\}$. Therefore, for each $i \in\{3,4\}$, Lemma 3.3 implies that

$$
d_{Y_{1}}\left(g_{1}\left(v_{1}\right), g_{1}\left(v_{i}\right)\right) \geq d_{1 i}
$$

because

$$
\begin{array}{ll}
d_{Y_{1}}\left(g_{1}\left(v_{5}\right), g_{1}\left(v_{1}\right)\right)=d_{51}, & d_{Y_{1}}\left(g_{1}\left(v_{1}\right), g_{1}\left(v_{2}\right)\right)=d_{12}, \quad d_{Y_{1}}\left(g_{1}\left(v_{2}\right), g_{1}\left(v_{i}\right)\right)=d_{2 i}, \\
d_{Y_{1}}\left(g_{1}\left(v_{i}\right), g_{1}\left(v_{5}\right)\right)=d_{i 5}, & d_{Y_{1}}\left(g_{1}\left(v_{2}\right), g_{1}\left(v_{5}\right)\right)=d_{25}
\end{array}
$$


by (8.17) and (8.18). By (8.17), (8.18) and (8.19),

$$
\begin{cases}d_{Y_{1}}\left(g_{1}\left(v_{i}\right), g_{1}\left(v_{j}\right)\right)=d_{i j}, & \text { if }\left\{v_{i}, v_{j}\right\} \in E, \\ d_{Y_{1}}\left(g_{1}\left(v_{i}\right), g_{1}\left(v_{j}\right)\right) \geq d_{i j}, & \text { if }\left\{v_{i}, v_{j}\right\} \notin E\end{cases}
$$

for any $i, j \in\{1,2,3,4,5\}$. Thus $g_{1}$ is a map from $V$ to a $\operatorname{CAT}(0)$ space with the desired properties.

CASE 2: $\left\{f\left(v_{2}\right), f\left(v_{3}\right), f\left(v_{4}\right), f\left(v_{5}\right)\right\}$ is under-distance with respect to $\left\{f\left(v_{2}\right), f\left(v_{5}\right)\right\}$. Choose $x_{2}, x_{3}, x_{4} \in \mathbb{R}^{2}$ such that

$$
\left\|x_{2}-x_{3}\right\|=d_{23}, \quad\left\|x_{3}-x_{4}\right\|=d_{34}, \quad\left\|x_{4}-x_{2}\right\|=d_{42} .
$$

Suppose $x_{5} \in \mathbb{R}^{2}$ is a point such that

$$
\left\|x_{3}-x_{5}\right\|=d_{35}, \quad\left\|x_{5}-x_{4}\right\|=d_{54},
$$

and $x_{5}$ is not on the opposite side of $\overleftrightarrow{x_{3} x_{4}}$ from $x_{2}$. Then the assumption of CASE 2 implies that

$$
d_{25}<\left\|x_{2}-x_{5}\right\|,
$$

and Corollary 7.6 implies that $x_{5} \in \operatorname{conv}\left(\left\{x_{3}, x_{4}, x_{2}\right\}\right)$ or $x_{2} \in \operatorname{conv}\left(\left\{x_{3}, x_{4}, x_{5}\right\}\right)$. By the symmetry of the graph $G_{7}^{(5)}(0)$, we may assume without loss of generality that

$$
x_{5} \in \operatorname{conv}\left(\left\{x_{3}, x_{4}, x_{2}\right\}\right) .
$$

Choose $y_{2}, y_{3}, y_{5} \in \mathbb{R}^{2}$ such that

$$
\left\|y_{2}-y_{3}\right\|=d_{23}, \quad\left\|y_{3}-y_{5}\right\|=d_{35}, \quad\left\|y_{5}-y_{2}\right\|=d_{52},
$$

and choose $y_{4} \in \mathbb{R}^{2}$ such that

$$
\left\|y_{2}-y_{4}\right\|=d_{24}, \quad\left\|y_{4}-y_{5}\right\|=d_{45} .
$$

Then because

$$
\begin{array}{ll}
\left\|y_{3}-y_{2}\right\|=d_{32}=\left\|x_{3}-x_{2}\right\|, & \left\|y_{2}-y_{4}\right\|=d_{24}=\left\|x_{2}-x_{4}\right\|, \\
\left\|y_{3}-y_{5}\right\|=d_{35}=\left\|x_{3}-x_{5}\right\|, & \left\|y_{5}-y_{4}\right\|=d_{54}=\left\|x_{5}-x_{4}\right\|, \\
\left\|y_{2}-y_{5}\right\|=d_{25}<\left\|x_{2}-x_{5}\right\|, & x_{5} \in \operatorname{conv}\left(\left\{x_{3}, x_{4}, x_{2}\right\}\right)
\end{array}
$$

by (8.20) and (8.21), Lemma 8.4 implies that

$$
\left\|y_{3}-y_{4}\right\| \leq\left\|x_{3}-x_{4}\right\|=d_{34} .
$$

Define $\left(Y_{2}, d_{Y_{2}}\right)$ to be the metric space obtained by gluing $\mathbb{R}^{2}$ and $P$ by identifying $\left[y_{2}, y_{5}\right] \subseteq \mathbb{R}^{2}$ with $\left[p_{2}, p_{5}\right] \subseteq P$. Then $\left(Y_{2}, d_{Y_{2}}\right)$ is a CAT(0) space by Reshetnyak's gluing theorem. Define a map $g_{2}: V \rightarrow Y_{2}$ by sending $v_{i}$ to the point in $Y_{2}$ represented by $y_{i} \in \mathbb{R}^{2}$ for each $i \in\{2,3,4,5\}$, and $v_{1}$ to the point in $Y_{2}$ represented by $p_{1} \in P$. Then

$$
\begin{aligned}
& d_{Y_{2}}\left(g_{2}\left(v_{1}\right), g_{2}\left(v_{i}\right)\right)=\left\|p_{1}-p_{i}\right\|=d_{1 i}, \\
& d_{Y_{2}}\left(g_{2}\left(v_{j}\right), g_{2}\left(v_{k}\right)\right)=\left\|y_{j}-y_{k}\right\|=d_{j k}
\end{aligned}
$$

for any $i \in\{2,5\}$ and any $j, k \in\{2,3,4,5\}$ with $\{j, k\} \neq\{3,4\}$. By (8.22),

$$
d_{Y_{2}}\left(g_{2}\left(v_{3}\right), g_{2}\left(v_{4}\right)\right)=\left\|y_{3}-y_{4}\right\| \leq d_{34} .
$$

It is clear from the definitions of $Y_{2}$ and $g_{2}$ that

$$
\begin{aligned}
& {\left[g_{2}\left(v_{2}\right), g_{2}\left(v_{5}\right)\right] \cap\left[g_{2}\left(v_{1}\right), g_{2}\left(v_{i}\right)\right] \neq \emptyset,} \\
& \operatorname{conv}\left(\left\{g_{2}\left(v_{1}\right), g_{2}\left(v_{2}\right), g_{2}\left(v_{5}\right)\right\}\right) \cap \operatorname{conv}\left(\left\{g_{2}\left(v_{i}\right), g_{2}\left(v_{2}\right), g_{2}\left(v_{5}\right)\right\}\right)=\left[g_{2}\left(v_{2}\right), g_{2}\left(v_{5}\right)\right]
\end{aligned}
$$


for each $i \in\{3,4\}$, and $\operatorname{conv}\left(\left\{g_{2}\left(v_{j}\right), g_{2}\left(v_{2}\right), g_{2}\left(v_{5}\right)\right\}\right)$ is isometric to a convex subset of the Euclidean plane for each $j \in\{1,3,4\}$. Therefore, for each $i \in\{3,4\}$, Lemma 3.3 implies that

$$
d_{Y_{2}}\left(g_{2}\left(v_{1}\right), g_{2}\left(v_{i}\right)\right) \geq d_{1 i}
$$

because

$$
\begin{aligned}
& d_{Y_{2}}\left(g_{2}\left(v_{5}\right), g_{2}\left(v_{1}\right)\right)=d_{51}, \quad d_{Y_{2}}\left(g_{2}\left(v_{1}\right), g_{2}\left(v_{2}\right)\right)=d_{12}, \quad d_{Y_{2}}\left(g_{2}\left(v_{2}\right), g_{2}\left(v_{i}\right)\right)=d_{2 i}, \\
& d_{Y_{2}}\left(g_{2}\left(v_{i}\right), g_{2}\left(v_{5}\right)\right)=d_{i 5}, \quad d_{Y_{2}}\left(g_{2}\left(v_{2}\right), g_{2}\left(v_{5}\right)\right)=d_{25}
\end{aligned}
$$

by (8.23) and (8.24). By (8.23), (8.24), (8.25) and (8.26),

$$
\begin{cases}d_{Y_{2}}\left(g_{2}\left(v_{i}\right), g_{2}\left(v_{j}\right)\right) \leq d_{i j}, & \text { if }\left\{v_{i}, v_{j}\right\} \in E, \\ d_{Y_{2}}\left(g_{2}\left(v_{i}\right), g_{2}\left(v_{j}\right)\right) \geq d_{i j}, & \text { if }\left\{v_{i}, v_{j}\right\} \notin E\end{cases}
$$

for any $i, j \in\{1,2,3,4,5\}$. Thus $g_{2}$ is a map from $V$ to a CAT(0) space with the desired properties.

CASE 3: $\left\{f\left(v_{2}\right), f\left(v_{3}\right), f\left(v_{4}\right), f\left(v_{5}\right)\right\}$ is over-distance with respect to $\left\{f\left(v_{2}\right), f\left(v_{5}\right)\right\}$. In this case, Lemma 7.7 implies that

$$
\pi<\tilde{Z} f\left(v_{2}\right) f\left(v_{3}\right) f\left(v_{4}\right)+\tilde{Z} f\left(v_{4}\right) f\left(v_{3}\right) f\left(v_{5}\right) .
$$

or

$$
\pi<\tilde{L} f\left(v_{2}\right) f\left(v_{4}\right) f\left(v_{3}\right)+\tilde{L} f\left(v_{3}\right) f\left(v_{4}\right) f\left(v_{5}\right),
$$

By the symmetry of the graph $G_{7}^{(5)}$, we may assume without loss of generality that the former inequality holds. Let $Y_{3}^{\prime}=D\left(f\left(v_{3}\right) ; f\left(v_{2}\right), f\left(v_{4}\right), f\left(v_{5}\right)\right)$, and let

$$
\psi:\left\{f\left(v_{3}\right), f\left(v_{2}\right), f\left(v_{4}\right), f\left(v_{5}\right)\right\} \rightarrow Y_{3}^{\prime}
$$

be the natural inclusion. Then $Y_{3}^{\prime}$ is a CAT(0) space, and $\psi$ is an isometric embedding by Lemma 7.14. It also follows from Lemma 7.14 that

$$
T\left(f\left(v_{3}\right), f\left(v_{2}\right), f\left(v_{4}\right)\right), \quad T\left(f\left(v_{3}\right), f\left(v_{4}\right), f\left(v_{5}\right)\right), \quad T\left(f\left(v_{3}\right), f\left(v_{5}\right), f\left(v_{2}\right)\right)
$$

are closed convex subsets of $Y_{3}^{\prime}$, all of which are isometric to convex subsets of the Euclidean plane. Define $\left(Y_{3}, d_{Y_{3}}\right)$ to be the metric space obtained by gluing $Y_{3}^{\prime}$ and $P$ by identifying $\left[\psi\left(f\left(v_{2}\right)\right), \psi\left(f\left(v_{5}\right)\right)\right] \subseteq Y_{3}^{\prime}$ with $\left[p_{2}, p_{5}\right] \subseteq P$. Then $\left(Y_{3}, d_{Y_{3}}\right)$ is a CAT(0) space by Reshetnyak's gluing theorem. Define a map $g_{3}: V \rightarrow Y_{3}$ by sending $v_{i}$ to the point in $Y_{3}$ represented by $\psi\left(f\left(v_{i}\right)\right) \in Y_{3}^{\prime}$ for each $i \in\{2,3,4,5\}$, and $v_{1}$ to the point in $Y_{3}$ represented by $p_{1} \in P$. Then

$$
\begin{aligned}
& d_{Y_{3}}\left(g_{3}\left(v_{1}\right), g_{3}\left(v_{i}\right)\right)=\left\|p_{1}-p_{i}\right\|=d_{1 i}, \\
& d_{Y_{3}}\left(g_{3}\left(v_{j}\right), g_{3}\left(v_{k}\right)\right)=d_{Y_{3}^{\prime}}\left(\psi\left(f\left(v_{j}\right)\right), \psi\left(f\left(v_{k}\right)\right)\right)=d_{j k}
\end{aligned}
$$

for any $i \in\{2,5\}$ and any $j, k \in\{2,3,4,5\}$. Let $T_{1}, T_{2}$ and $T_{3}$ be the images of $T\left(f\left(v_{3}\right), f\left(v_{2}\right), f\left(v_{4}\right)\right)$, $T\left(f\left(v_{3}\right), f\left(v_{4}\right), f\left(v_{5}\right)\right)$ and $T\left(f\left(v_{3}\right), f\left(v_{5}\right), f\left(v_{2}\right)\right)$, respectively under the natural inclusion of $Y_{3}^{\prime}$ into $Y_{3}$, and let $\tilde{P}$ be the image of $P$ under the natural inclusion of $P$ into $Y_{3}$. Then it is clear from the definition of $Y_{3}$ that $T_{1}, T_{2}, T_{3}$ and $\tilde{P}$ are all isometric to convex subsets of the Euclidean plane, and

$$
T_{1} \cap T_{3}=\left[g_{3}\left(v_{3}\right), g_{3}\left(v_{2}\right)\right], \quad T_{2} \cap T_{3}=\left[g_{3}\left(v_{3}\right), g_{3}\left(v_{5}\right)\right], \quad T_{3} \cap \tilde{P}=\left[g_{3}\left(v_{2}\right), g_{3}\left(v_{5}\right)\right] .
$$

It is also clear from the definition of $Y_{3}$ that there exist $q_{0}, q_{1} \in\left[g_{3}\left(v_{2}\right), g_{3}\left(v_{5}\right)\right]$ such that

$$
\begin{aligned}
& d_{Y_{3}}\left(g_{3}\left(v_{1}\right), g_{3}\left(v_{3}\right)\right)=d_{Y_{3}}\left(g_{3}\left(v_{1}\right), q_{0}\right)+d_{Y_{3}}\left(q_{0}, g_{3}\left(v_{3}\right)\right), \\
& d_{Y_{3}}\left(g_{3}\left(v_{1}\right), g_{3}\left(v_{4}\right)\right)=d_{Y_{3}}\left(g_{3}\left(v_{1}\right), q_{1}\right)+d_{Y_{3}}\left(q_{1}, g_{3}\left(v_{4}\right)\right) .
\end{aligned}
$$

Therefore, (8.29) and Lemma 3.3 imply that

$$
d_{Y_{3}}\left(g_{3}\left(v_{1}\right), g_{3}\left(v_{3}\right)\right) \geq d_{13}
$$


because

$$
\begin{array}{lll}
d_{Y_{3}}\left(g_{3}\left(v_{2}\right), g_{3}\left(v_{1}\right)\right)=d_{21}, & d_{Y_{3}}\left(g_{3}\left(v_{1}\right), g_{3}\left(v_{5}\right)\right)=d_{15}, \quad d_{Y_{3}}\left(g_{3}\left(v_{5}\right), g_{3}\left(v_{3}\right)\right)=d_{53}, \\
d_{Y_{3}}\left(g_{3}\left(v_{3}\right), g_{3}\left(v_{2}\right)\right)=d_{32}, & d_{Y_{3}}\left(g_{3}\left(v_{2}\right), g_{3}\left(v_{5}\right)\right)=d_{25}
\end{array}
$$

by (8.27) and (8.28). Clearly, the point $q_{1} \in\left[g_{3}\left(v_{2}\right), g_{3}\left(v_{5}\right)\right]$ is represented by a point $q_{1}^{\prime} \in\left[\psi\left(f\left(v_{2}\right)\right), \psi\left(f\left(v_{5}\right)\right)\right]$, and by definition of $Y_{3}^{\prime}=D\left(f\left(v_{3}\right) ; f\left(v_{2}\right), f\left(v_{4}\right), f\left(v_{5}\right)\right)$, there exists $q_{2}^{\prime} \in\left[\psi\left(f\left(v_{3}\right)\right), \psi\left(f\left(v_{2}\right)\right)\right] \cup$ $\left[\psi\left(f\left(v_{3}\right)\right), \psi\left(f\left(v_{5}\right)\right)\right]$ such that

$$
d_{Y_{3}}\left(q_{1}, g_{3}\left(v_{4}\right)\right)=d_{Y_{3}^{\prime}}\left(q_{1}^{\prime}, \psi\left(f\left(v_{4}\right)\right)\right)=d_{Y_{3}^{\prime}}\left(q_{1}^{\prime}, q_{2}^{\prime}\right)+d_{Y_{3}^{\prime}}\left(q_{2}^{\prime}, \psi\left(f\left(v_{4}\right)\right)\right) .
$$

It follows from (8.30) and (8.32) that

$$
d_{Y_{3}}\left(g_{3}\left(v_{1}\right), g_{3}\left(v_{4}\right)\right)=d_{Y_{3}}\left(g_{3}\left(v_{1}\right), q_{1}\right)+d_{Y_{3}}\left(q_{1}, q_{2}\right)+d_{Y_{3}}\left(q_{2}, g_{3}\left(v_{4}\right)\right),
$$

where $q_{2} \in Y_{3}$ is the point represented by $q_{2}^{\prime} \in Y_{3}^{\prime}$. If $q_{2}^{\prime} \in\left[\psi\left(f\left(v_{3}\right)\right), \psi\left(f\left(v_{2}\right)\right)\right]$, then clearly $q_{2} \in$ $\left[g_{3}\left(v_{3}\right), g_{3}\left(v_{2}\right)\right]$, and therefore (8.33) and Lemma 6.5 imply that

$$
d_{Y_{3}}\left(g_{3}\left(v_{1}\right), g_{3}\left(v_{4}\right)\right) \geq d_{14}
$$

because

$$
\begin{array}{lll}
d_{Y_{3}}\left(g_{3}\left(v_{2}\right), g_{3}\left(v_{1}\right)\right)=d_{21}, & d_{Y_{3}}\left(g_{3}\left(v_{1}\right), g_{3}\left(v_{5}\right)\right)=d_{15}, & d_{Y_{3}}\left(g_{3}\left(v_{5}\right), g_{3}\left(v_{3}\right)\right)=d_{53}, \\
d_{Y_{3}}\left(g_{3}\left(v_{3}\right), g_{3}\left(v_{4}\right)\right)=d_{34}, & d_{Y_{3}}\left(g_{3}\left(v_{4}\right), g_{3}\left(v_{2}\right)\right)=d_{42}, & d_{Y_{3}}\left(g_{3}\left(v_{2}\right), g_{3}\left(v_{5}\right)\right)=d_{25}, \\
d_{Y_{3}}\left(g_{3}\left(v_{2}\right), g_{3}\left(v_{3}\right)\right)=d_{23} &
\end{array}
$$

by (8.27) and (8.28). If $q_{2}^{\prime} \in\left[\psi\left(f\left(v_{3}\right)\right), \psi\left(f\left(v_{5}\right)\right)\right]$, then we obtain (8.34) in the same way. By (8.27), (8.28), (8.31) and (8.34),

$$
\begin{cases}d_{Y_{3}}\left(g_{3}\left(v_{i}\right), g_{3}\left(v_{j}\right)\right)=d_{i j}, & \text { if }\left\{v_{i}, v_{j}\right\} \in E, \\ d_{Y_{3}}\left(g_{3}\left(v_{i}\right), g_{3}\left(v_{j}\right)\right) \geq d_{i j}, & \text { if }\left\{v_{i}, v_{j}\right\} \notin E\end{cases}
$$

for any $i, j \in\{1,2,3,4,5\}$. Thus $g_{3}$ is a map from $V$ to a $\operatorname{CAT}(0)$ space with the desired properties.

By Proposition 7.2, CASE 1, CASE 2 and CASE 3 exhaust all possibilities.

\section{The $G_{9}^{(5)}(0)$ condition}

In this section, we prove that the validity of the $\triangle$-inequalities implies the $G_{9}^{(5)}(0)$ condition. First we prove several lemmas.

Lemma 9.1. Let $\left(X, d_{X}\right)$ be a metric space that satisfies the $\otimes$-inequalities, and let $p, x, y, z, w \in X$. Suppose there exist a complete geodesic space with nonnegative Alexandrov curvature $\left(Z, d_{Z}\right)$ and a map $f$ : $\{p, x, y, z, w\} \rightarrow Z$ such that

$$
d_{Z}(f(p), f(a)) \leq d_{X}(p, a), \quad d_{Z}(f(a), f(b)) \geq d_{X}(a, b)
$$

for any $a, b \in\{x, y, z, w\}$. Then there exist a $\operatorname{CAT}(0)$ space $\left(Y, d_{Y}\right)$ and a map $g:\{p, x, y, z, w\} \rightarrow Y$ such that

$$
d_{Y}(g(p), g(a)) \leq d_{X}(p, a), \quad d_{Y}(g(a), g(b))=d_{X}(a, b)
$$

for any $a, b \in\{x, y, z, w\}$.

Proof. Because $\left(X, d_{X}\right)$ satisfies the $\otimes$-inequalities, Theorem 1.7 implies that there exist a CAT $(0)$ space $\left(Y, d_{Y}\right)$ and an isometric embedding $\varphi:\{x, y, z, w\} \rightarrow Y$. Define a map $\psi:\{f(x), f(y), f(z), f(w)\} \rightarrow Y$ by $\psi(f(a))=$ $\varphi(a)$. Then $\psi$ is 1 -Lipschitz because

$$
d_{Y}(\psi(f(a)), \psi(f(b)))=d_{Y}(\varphi(a), \varphi(b))=d_{X}(a, b) \leq d_{Z}(f(a), f(b))
$$


for any $a, b \in\{x, y, z, w\}$. Hence Theorem 2.6 implies that there exists a 1-Lipschitz map $\tilde{\psi}$ : $\{f(p), f(x), f(y), f(z), f(w)\} \rightarrow Y$ such that $\tilde{\psi}(f(a))=\psi(f(a))$ for every $a \in\{x, y, z, w\}$. Define a map $g:\{p, x, y, z, w\} \rightarrow Y$ by $g(a)=\tilde{\psi}(f(a))$. Then

$$
\begin{aligned}
& d_{Y}(g(a), g(b))=d_{Y}(\psi(f(a)), \psi(f(b)))=d_{Y}(\varphi(a), \varphi(b))=d_{X}(a, b), \\
& d_{Y}(g(p), g(a))=d_{Y}(\tilde{\psi}(f(p)), \tilde{\psi}(f(a))) \leq d_{Z}(f(p), f(a)) \leq d_{X}(p, a)
\end{aligned}
$$

for any $a, b \in\{x, y, z, w\}$, which proves the lemma.

Lemma 9.2. Let $\left(X, d_{X}\right)$ be a metric space that satisfies the $\bigotimes$-inequalities. Suppose $p, q, x, y, z \in X$ are five distinct points such that both $\{p, x, y, z\}$ and $\{q, x, y, z\}$ admit isometric embeddings into $\mathbb{R}^{3}$. Then there exist $a \operatorname{CAT}(0)$ space $\left(Y, d_{Y}\right)$ and a map $g:\{p, q, x, y, z\} \rightarrow Y$ such that

$$
d_{Y}(g(p), g(q)) \geq d_{X}(p, q), \quad d_{Y}(g(x), g(a)) \leq d_{X}(x, a), \quad d_{Y}(g(b), g(c))=d_{X}(b, c)
$$

for any $a, b, c \in\{p, q, y, z\}$ with $\{b, c\} \neq\{p, q\}$.

Proof. Let $\alpha$ be the plane in $\mathbb{R}^{3}$ consisting of all points $\left(t_{1}, t_{2}, t_{3}\right) \in \mathbb{R}^{3}$ with $t_{3}=0$. Choose $x^{\prime}, y^{\prime}, z^{\prime} \in \alpha$ such that

$$
\left\|x^{\prime}-y^{\prime}\right\|=d_{X}(x, y), \quad\left\|y^{\prime}-z^{\prime}\right\|=d_{X}(y, z), \quad\left\|z^{\prime}-x^{\prime}\right\|=d_{X}(z, x) .
$$

Then because both $\{p, x, y, z\}$ and $\{q, x, y, z\}$ admit isometric embeddings into $\mathbb{R}^{3}$, there exist points $p^{\prime}=$ $\left(p^{(1)}, p^{(2)}, p^{(3)}\right)$ and $q^{\prime}=\left(q^{(1)}, q^{(2)}, q^{(3)}\right)$ in $\mathbb{R}^{3}$ such that

$$
\begin{array}{llll}
\left\|p^{\prime}-x^{\prime}\right\|=d_{X}(p, x), & \left\|p^{\prime}-y^{\prime}\right\|=d_{X}(p, y), & \left\|p^{\prime}-z^{\prime}\right\|=d_{X}(p, z), & p^{(3)} \geq 0, \\
\left\|q^{\prime}-x^{\prime}\right\|=d_{X}(q, x), & \left\|q^{\prime}-y^{\prime}\right\|=d_{X}(q, y), & \left\|q^{\prime}-z^{\prime}\right\|=d_{X}(q, z), & q^{(3)} \leq 0 .
\end{array}
$$

Let $R$ be the convex hull of $\left\{x^{\prime}, y^{\prime}, z^{\prime}\right\}$ in $\mathbb{R}^{3}$. Then $R \subseteq \alpha$, and the triangle

$$
R^{\prime}=\left[x^{\prime}, y^{\prime}\right] \cup\left[y^{\prime}, z^{\prime}\right] \cup\left[z^{\prime}, x^{\prime}\right]
$$

forms the boundary of $R$ as a subset of $\alpha$. Define $P, Q \subseteq \mathbb{R}^{3}$ by

$$
P=\operatorname{conv}\left(\left\{p^{\prime}, x^{\prime}, y^{\prime}, z^{\prime}\right\}\right), \quad Q=\operatorname{conv}\left(\left\{q^{\prime}, x^{\prime}, y^{\prime}, z^{\prime}\right\}\right) .
$$

We consider three cases.

CASE 1: $\left[p^{\prime}, q^{\prime}\right] \cap\left(\alpha \backslash\left(R \backslash R^{\prime}\right)\right) \neq \emptyset$. Choose $r_{0} \in\left[p^{\prime}, q^{\prime}\right] \cap\left(\alpha \backslash\left(R \backslash R^{\prime}\right)\right)$. Equip the subsets $P$ and $Q$ of $\mathbb{R}^{3}$ with the induced metrics, and regard them as disjoint metric spaces. Define $\left(Y_{1}, d_{Y_{1}}\right)$ to be the metric space obtained by gluing $P$ and $Q$ by identifying $R \subseteq P$ with $R \subseteq Q$ naturally. Then $Y_{1}$ is a CAT(0) space by Reshetnyak's gluing theorem, and the natural inclusions of $P$ and $Q$ into $Y_{1}$ are isometric embeddings. We denote by $\tilde{P}$ and $\tilde{Q}$ the images of $P$ and $Q$, respectively under the natural inclusions into $Y_{1}$. Define a map $g_{1}:\{p, q, x, y, z\} \rightarrow Y_{1}$ by sending $x, y, z, p$ and $q$ to the points in $Y_{1}$ represented by $x^{\prime}, y^{\prime}, z^{\prime}, p^{\prime} \in P$ and $q^{\prime} \in Q$, respectively. Then

$$
d_{Y_{1}}\left(g_{1}(a), g_{1}(b)\right)=\left\|a^{\prime}-b^{\prime}\right\|=d_{X}(a, b)
$$

for any $a, b \in\{p, q, x, y, z\}$ with $\{a, b\} \neq\{p, q\}$. By definition of $Y_{1}$, there exists a point $r_{1} \in R$ such that

$$
d_{Y_{1}}\left(g_{1}(p), g_{1}(q)\right)=\left\|p^{\prime}-r_{1}\right\|+\left\|r_{1}-q^{\prime}\right\| .
$$

Because $R^{\prime}$ is the boundary of $R$ as a subset of $\alpha$, there exists a point $r_{2} \in R^{\prime} \cap\left[r_{0}, r_{1}\right]$. Then $r_{2} \in$ $\operatorname{conv}\left(\left\{r_{1}, p^{\prime}, q^{\prime}\right\}\right)$, and therefore Lemma 8.1 implies that

$$
d_{Y_{1}}\left(g_{1}(p), \tilde{r}_{2}\right)+d_{Y_{1}}\left(\tilde{r}_{2}, g_{1}(q)\right)=\left\|p^{\prime}-r_{2}\right\|+\left\|r_{2}-q^{\prime}\right\| \leq\left\|p^{\prime}-r_{1}\right\|+\left\|r_{1}-q^{\prime}\right\|,
$$

where $\tilde{r}_{2}$ is the point in $Y_{1}$ represented by $r_{2} \in P$ (or $r_{2} \in Q$ ). Combining this with (9.2) and the triangle inequality for $Y_{1}$ yields

$$
d_{Y_{1}}\left(g_{1}(p), g_{1}(q)\right)=d_{Y_{1}}\left(g_{1}(p), \tilde{r}_{2}\right)+d_{Y_{1}}\left(\tilde{r}_{2}, g_{1}(q)\right) \text {. }
$$


If $r_{2} \in\left[x^{\prime}, y^{\prime}\right]$, then $\tilde{r}_{2}$ clearly lies on the geodesic segment $\left[g_{1}(x), g_{1}(y)\right]$ in $Y_{1}$, and therefore (9.1), (9.3) and Lemma 3.3 imply that

$$
d_{Y_{1}}\left(g_{1}(p), g_{1}(q)\right) \geq d_{X}(p, q)
$$

because $\left[g_{1}(x), g_{1}(y)\right] \subseteq \tilde{P} \cup \tilde{Q}$, and $\tilde{P}$ and $\tilde{Q}$ are isometric to convex subsets of Euclidean spaces. If $r_{2} \in\left[y^{\prime}, z^{\prime}\right]$ or $r_{2} \in\left[z^{\prime}, x^{\prime}\right]$, then we obtain (9.4) in the same way. Thus (9.4) always holds in CASE 1. By (9.1) and (9.4), $g_{1}$ is a map from $\{p, q, x, y, z\}$ to a CAT(0) space with the desired properties.

CASE 2: $\left[p^{\prime}, q^{\prime}\right] \cap\left(\alpha \backslash\left(R \backslash R^{\prime}\right)\right)=\emptyset$ and $\left\{p^{\prime}, q^{\prime}\right\} q \alpha$. In this case, $\left[p^{\prime}, q^{\prime}\right] \cap\left(R \backslash R^{\prime}\right) \neq \emptyset$. Hence the subset $P \cup Q$ of $\mathbb{R}^{3}$ is not contained in any plane, and $\left[p^{\prime}, q^{\prime}\right] \subseteq P \cup Q$. It follows that $P \cup Q$ is a convex subset of $\mathbb{R}^{3}$, and therefore the boundary $S$ of $P \cup Q$ in $\mathbb{R}^{3}$ equipped with the induced length metric $d_{S}$ is a complete geodesic space with nonnegative Alexandrov curvature as we mentioned in Example 2.4. Clearly $S$ is the union of six subsets $\operatorname{conv}\left(\left\{p^{\prime}, x^{\prime}, y^{\prime}\right\}\right), \operatorname{conv}\left(\left\{p^{\prime}, y^{\prime}, z^{\prime}\right\}\right), \operatorname{conv}\left(\left\{p^{\prime}, z^{\prime}, x^{\prime}\right\}\right), \operatorname{conv}\left(\left\{q^{\prime}, x^{\prime}, y^{\prime}\right\}\right), \operatorname{conv}\left(\left\{q^{\prime}, y^{\prime}, z^{\prime}\right\}\right)$ and $\operatorname{conv}\left(\left\{q^{\prime}, z^{\prime}, x^{\prime}\right\}\right)$ of $\mathbb{R}^{3}$. On each of these six subsets, $d_{S}$ coincides with the Euclidean metric on $\mathbb{R}^{3}$. In particular, these six subsets are all isometric to convex subsets of the Euclidean plane even as subsets of $\left(S, d_{S}\right)$. Define a map $f_{2}:\{p, q, x, y, z\} \rightarrow S$ by $f_{2}(a)=a^{\prime}$. Then

$$
d_{S}\left(f_{2}(a), f_{2}(b)\right)=\left\|a^{\prime}-b^{\prime}\right\|=d_{X}(a, b)
$$

for any $a, b \in\{p, q, x, y, z\}$ with $\{a, b\} \neq\{p, q\}$. Fix a geodesic segment $\Gamma_{0}$ in $\left(S, d_{S}\right)$ with endpoints $f_{2}(p)$ and $f_{2}(q)$. Then $\Gamma_{0}$ clearly has a nonempty intersection with the union of three line segments $\left[x^{\prime}, y^{\prime}\right] \cup\left[y^{\prime}, z^{\prime}\right] \cup$ $\left[z^{\prime}, x^{\prime}\right]$. If $\Gamma_{0}$ has a nonempty intersection with $\left[x^{\prime}, y^{\prime}\right]$, then (9.5) and Lemma 3.3 imply that

$$
d_{S}\left(f_{2}(p), f_{2}(q)\right) \geq d_{X}(p, q)
$$

because $\left[x^{\prime}, y^{\prime}\right]=\operatorname{conv}\left(\left\{p^{\prime}, x^{\prime}, y^{\prime}\right\}\right) \cap \operatorname{conv}\left(\left\{q^{\prime}, x^{\prime}, y^{\prime}\right\}\right)$ is a geodesic segment even in $\left(S, d_{S}\right)$. If $\Gamma_{0}$ has a nonempty intersection with $\left[y^{\prime}, z^{\prime}\right]$ or $\left[z^{\prime}, x^{\prime}\right]$, then we obtain (9.6) in the same way. Thus (9.6) always holds in CASE 2. By (9.5) and (9.6), the map $f_{2}$ satisfies that

$$
d_{S}\left(f_{2}(x), f_{2}(a)\right)=d_{X}(x, a), \quad d_{S}\left(f_{2}(a), f_{2}(b)\right) \geq d_{X}(a, b)
$$

for any $a, b \in\{p, q, y, z\}$. Therefore, Lemma 9.1 implies that there exist a CAT(0) space $\left(Y_{2}, d_{Y_{2}}\right)$ and a map $g_{2}:\{p, q, x, y, z\} \rightarrow Y_{2}$ such that

$$
d_{Y_{2}}\left(g_{2}(x), g_{2}(a)\right) \leq d_{X}(x, a), \quad d_{Y_{2}}\left(g_{2}(a), g_{2}(b)\right)=d_{X}(a, b)
$$

for any $a, b \in\{p, q, y, z\}$. Thus $g_{2}$ is a map from $\{p, q, x, y, z\}$ to a CAT(0) space with the desired properties.

CASE 3: $\left[p^{\prime}, q^{\prime}\right] \cap\left(\alpha \backslash\left(R \backslash R^{\prime}\right)\right)=\emptyset$ and $\left\{p^{\prime}, q^{\prime}\right\} \subseteq \alpha$. In this case, $\left\{p^{\prime}, q^{\prime}\right\} \subseteq R \backslash R^{\prime}$, which ensures in particular that $x^{\prime}, y^{\prime}$ and $z^{\prime}$ are not collinear. Let $R_{1}$ and $R_{2}$ be two isometric copies of $R$. We denote the points in $R_{1}$ corresponding to $x^{\prime}, y^{\prime}, z^{\prime}, p^{\prime}$ and $q^{\prime}$ by $x_{1}, y_{1}, z_{1}, p_{1}$ and $q_{1}$, respectively, and the points in $R_{2}$ corresponding to $x^{\prime}, y^{\prime}, z^{\prime}, p^{\prime}$ and $q^{\prime}$ by $x_{2}, y_{2}, z_{2}, p_{2}$ and $q_{2}$, respectively. Define $\left(R_{0}, d_{R_{0}}\right)$ to be the piecewise Euclidean simplicial complex constructed from the two simplices $R_{1}$ and $R_{2}$ by identifying $\left[x_{1}, y_{1}\right]$ with $\left[x_{2}, y_{2}\right],\left[y_{1}, z_{1}\right]$ with $\left[y_{2}, z_{2}\right]$, and $\left[z_{1}, x_{1}\right]$ with $\left[z_{2}, x_{2}\right]$. In other words, $R_{0}$ is the piecewise Euclidean simplicial complex obtained by gluing $R_{1}$ and $R_{2}$ along their boundaries. As we mentioned in Example 2.5, $R_{0}$ is a complete geodesic space with nonnegative Alexandrov curvature, and the natural inclusions of $R_{1}$ and $R_{2}$ into $R_{0}$ are both isometric embeddings. In particular, for each $i \in\{1,2\}$, the image $\tilde{R}_{i}$ of $R_{i}$ under the natural inclusion into $R_{0}$ is isometric to a convex subset of the Euclidean plane. Define a map $f_{3}:\{p, q, x, y, z\} \rightarrow R_{0}$ by sending $x, y, z, p$ and $q$ to the points in $R_{0}$ represented by $x_{1}, y_{1}, z_{1}, p_{1} \in R_{1}$ and $q_{2} \in R_{2}$, respectively. Then

$$
d_{R_{0}}\left(f_{3}(a), f_{3}(b)\right)=\left\|a^{\prime}-b^{\prime}\right\|=d_{X}(a, b)
$$

for any $a, b \in\{p, q, x, y, z\}$ with $\{a, b\} \neq\{p, q\}$. It follows from the definition of $R_{0}$ that there exists a point $r_{3} \in R^{\prime}$ such that

$$
d_{R_{0}}\left(f_{3}(p), f_{3}(q)\right)=\left\|p^{\prime}-r_{3}\right\|+\left\|r_{3}-q^{\prime}\right\|
$$

Hence

$$
d_{R_{0}}\left(f_{3}(p), f_{3}(q)\right)=d_{R_{0}}\left(f_{3}(p), \tilde{r}_{3}\right)+d_{R_{0}}\left(\tilde{r}_{3}, f_{3}(q)\right)
$$


where $\tilde{r}_{3}$ is the point in $R_{0}$ represented by the point in $R_{1}$ (or $R_{2}$ ) corresponding to $r_{3}$. Let $\Gamma_{1}$ be the image of the line segment $\left[x_{1}, y_{1}\right] \subseteq R_{1}$ (or $\left[x_{2}, y_{2}\right] \subseteq R_{2}$ ) under the natural inclusion into $R_{0}$. If $r_{3} \in\left[x^{\prime}, y^{\prime}\right]$, then $\tilde{r}_{3} \in \Gamma_{1}$, and therefore (9.7), (9.8) and Lemma 3.3 imply that

$$
d_{R_{0}}\left(f_{3}(p), f_{3}(q)\right) \geq d_{X}(p, q)
$$

because it is clear from the definition of $R_{0}$ that $\Gamma_{1}$ is a geodesic segment in $R_{0}$ with endpoints $f_{3}(x)$ and $f_{3}(y)$, and $\Gamma_{1} \subseteq \tilde{R}_{1} \cup \tilde{R}_{2}$. If $r_{3} \in\left[y^{\prime}, z^{\prime}\right]$ or $r_{3} \in\left[z^{\prime}, x^{\prime}\right]$, then we obtain (9.9) in the same way. Thus (9.9) always holds in CASE 3. By (9.7) and (9.9),

$$
d_{R_{0}}\left(f_{3}(x), f_{3}(a)\right)=d_{X}(x, a), \quad d_{R_{0}}\left(f_{3}(a), f_{3}(b)\right) \geq d_{X}(a, b)
$$

for any $a, b \in\{p, q, y, z\}$. Therefore, Lemma 9.1 implies that there exist a CAT(0) space $\left(Y_{3}, d_{Y_{3}}\right)$ and a map $g_{3}:\{p, q, x, y, z\} \rightarrow Y_{3}$ such that

$$
d_{Y_{3}}\left(g_{3}(x), g_{3}(a)\right) \leq d_{X}(x, a), \quad d_{Y_{3}}\left(g_{3}(a), g_{3}(b)\right)=d_{X}(a, b)
$$

for any $a, b \in\{p, q, y, z\}$. Thus $g_{3}$ is a map from $\{p, q, x, y, z\}$ to a CAT(0) space with the desired properties.

CASE 1, CASE 2 and CASE 3 exhaust all possibilities.

Lemma 9.3. Let $\left(X, d_{X}\right)$ be a metric space that satisfies the $\bigotimes$-inequalities. Suppose $p, q, x, y, z \in X$ are five distinct points such that $\{p, x, y, z\}$ is over-distance with respect to $\{p, x\},\{p, y\}$ or $\{p, z\}$, and $\{q, x, y, z\}$ is over-distance with respect to $\{q, x\},\{q, y\}$ or $\{q, z\}$. Then there exist a $\operatorname{CAT}(0)$ space $\left(Y, d_{Y}\right)$ and a map $g:\{p, q, x, y, z\} \rightarrow Y$ such that

$$
d_{Y}(g(p), g(q)) \geq d_{X}(p, q), \quad d_{Y}(g(a), g(b))=d_{X}(a, b)
$$

for any $a, b \in\{p, q, x, y, z\}$ with $\{a, b\} \neq\{p, q\}$.

Proof. By the hypothesis, we can choose $a_{1}, a_{2}, a_{3}, b_{1}, b_{2}, b_{3} \in\{x, y, z\}$ with

$$
\left\{a_{1}, a_{2}, a_{3}\right\}=\left\{b_{1}, b_{2}, b_{3}\right\}=\{x, y, z\}
$$

such that $\{p, x, y, z\}$ is over-distance with respect to $\left\{p, a_{2}\right\}$, and $\{q, x, y, z\}$ is over-distance with respect to $\left\{q, b_{2}\right\}$. Then Lemma 7.7 implies that $\pi<\tilde{L} a_{2} a_{1} a_{3}+\tilde{Z} a_{3} a_{1} p$, or $\pi<\tilde{Z} a_{2} a_{3} a_{1}+\tilde{L} a_{1} a_{3} p$, and that $\pi<$ $\tilde{Z} b_{2} b_{1} b_{3}+\tilde{Z} b_{3} b_{1} q$, or $\pi<\tilde{Z} b_{2} b_{3} b_{1}+\tilde{L} b_{1} b_{3} q$. Therefore, renaming the points if necessary, we may assume further that

$$
\pi<\tilde{L} a_{2} a_{1} a_{3}+\tilde{L} a_{3} a_{1} p, \quad \pi<\tilde{Z} b_{2} b_{1} b_{3}+\tilde{L} b_{3} b_{1} q .
$$

Let $Y_{1}=D\left(a_{1} ; a_{2}, a_{3}, p\right)$, and let $Y_{2}=D\left(b_{1} ; b_{2}, b_{3}, q\right)$. Suppose $\varphi_{1}:\{p, x, y, z\} \rightarrow Y_{1}$ and $\varphi_{2}:$ $\{q, x, y, z\} \rightarrow Y_{2}$ are the natural inclusions. Then Lemma 7.14 implies that $Y_{1}$ and $Y_{2}$ are CAT(0) spaces, and $\varphi_{1}$ and $\varphi_{2}$ are isometric embeddings. It also follows from Lemma 7.14 that

$$
S_{1}=T_{Y_{1}}\left(a_{1}, a_{2}, a_{3}\right), \quad S_{2}=T_{Y_{1}}\left(a_{1}, a_{3}, p\right), \quad S_{3}=T_{Y_{1}}\left(a_{1}, p, a_{2}\right)
$$

are closed convex subsets of $Y_{1}$, all of which are isometric to convex subsets of the Euclidean plane. Similarly,

$$
T_{1}=T_{Y_{2}}\left(b_{1}, b_{2}, b_{3}\right), \quad T_{2}=T_{Y_{2}}\left(b_{1}, b_{3}, q\right), \quad T_{3}=T_{Y_{2}}\left(b_{1}, q, a_{2}\right)
$$

are convex subsets of of $Y_{2}$, all of which are isometric to convex subsets of the Euclidean plane. By (9.10), $S_{1}$ and $T_{1}$ are isometric via the isometry $h: S_{1} \rightarrow T_{1}$ such that

$$
h\left(\varphi_{1}(x)\right)=\varphi_{2}(x), \quad h\left(\varphi_{1}(y)\right)=\varphi_{2}(y), \quad h\left(\varphi_{1}(z)\right)=\varphi_{2}(z) .
$$

We define a metric space $\left(Y, d_{Y}\right)$ to be the gluing of $Y_{1}$ and $Y_{2}$ along $h$. Then $Y$ is a CAT(0) space by Reshetnyak's gluing theorem, and the natural inclusions of $Y_{1}$ and $Y_{2}$ into $Y$ are both isometric embeddings. In particular, the images $\tilde{S}_{1}, \tilde{S}_{2}$ and $\tilde{S}_{3}$ of $S_{1}, S_{2}$ and $S_{3}$, respectively under the natural inclusion of $Y_{1}$ into $Y$, 
and the images $\tilde{T}_{1}, \tilde{T}_{2}$ and $\tilde{T}_{3}$ of $T_{1}, T_{2}$ and $T_{3}$, respectively under the natural inclusion of $Y_{2}$ into $Y$ are all isometric to convex subsets of the Euclidean plane. Define a map $g:\{p, q, x, y, z\} \rightarrow Y$ by sending each $a \in\{p, x, y, z\}$ to the point in $Y$ represented by $\varphi_{1}(a) \in Y_{1}$, and $q$ to the point in $Y$ represented by $\varphi_{2}(q) \in Y_{2}$. Then clearly

$$
d_{Y}(g(a), g(b))=d_{X}(a, b)
$$

for any $a, b \in\{p, q, x, y, z\}$ with $\{a, b\} \neq\{p, q\}$. By definition of $Y$, there exists $c_{0} \in S_{1}$ such that

$$
d_{Y}(g(p), g(q))=d_{Y_{1}}\left(\varphi_{1}(p), c_{0}\right)+d_{Y_{2}}\left(h\left(c_{0}\right), \varphi_{2}(q)\right) .
$$

It is clear from the definitions of $Y_{1}=D\left(a_{1} ; a_{2}, a_{3}, p\right)$ and $Y_{2}=D\left(b_{1} ; b_{2}, b_{3}, q\right)$ that there exist $i, j \in\{2,3\}$, $c_{1} \in\left[\varphi_{1}\left(a_{1}\right), \varphi_{1}\left(a_{i}\right)\right]$ and $c_{2} \in\left[\varphi_{2}\left(b_{1}\right), \varphi_{2}\left(b_{j}\right)\right]$ such that

$$
\begin{gathered}
d_{Y_{1}}\left(\varphi_{1}(p), c_{0}\right)=d_{Y_{1}}\left(\varphi_{1}(p), c_{1}\right)+d_{Y_{1}}\left(c_{1}, c_{0}\right), \\
d_{Y_{2}}\left(h\left(c_{0}\right), \varphi_{2}(q)\right)=d_{Y_{2}}\left(h\left(c_{0}\right), c_{2}\right)+d_{Y_{2}}\left(c_{2}, \varphi_{2}(q)\right) .
\end{gathered}
$$

It follows from (9.12), (9.13), (9.14) and the triangle inequality for $Y$ that

$$
\begin{aligned}
d_{Y}(g(p), g(q)) & =d_{Y_{1}}\left(\varphi_{1}(p), c_{1}\right)+d_{Y_{1}}\left(c_{1}, c_{0}\right)+d_{Y_{2}}\left(h\left(c_{0}\right), c_{2}\right)+d_{Y_{2}}\left(c_{2}, \varphi_{2}(q)\right) \\
& =d_{Y}\left(g(p), \tilde{c}_{1}\right)+d_{Y}\left(\tilde{c}_{1}, \tilde{c}_{0}\right)+d_{Y}\left(\tilde{c}_{0}, \tilde{c}_{2}\right)+d_{Y}\left(\tilde{c}_{2}, g(q)\right) \\
& =d_{Y}\left(g(p), \tilde{c}_{1}\right)+d_{Y}\left(\tilde{c}_{1}, \tilde{c}_{2}\right)+d_{Y}\left(\tilde{c}_{2}, g(q)\right),
\end{aligned}
$$

where $\tilde{c}_{0}, \tilde{c}_{1}$ and $\tilde{c}_{2}$ are the points in $Y$ represented by $c_{0}, c_{1} \in Y_{1}$ and $c_{2} \in Y_{2}$, respectively. Because the geodesic segments $\left[g\left(a_{1}\right), g\left(a_{i}\right)\right]$ and $\left[g\left(b_{1}\right), g\left(b_{j}\right)\right]$ in $Y$ are clearly the image of $\left[\varphi_{1}\left(a_{1}\right), \varphi_{1}\left(a_{i}\right)\right]$ under the natural inclusion of $Y_{1}$ into $Y$ and that of $\left[\varphi_{2}\left(b_{1}\right), \varphi_{2}\left(b_{j}\right)\right]$ under the natural inclusion of $Y_{2}$ into $Y$, respectively,

$$
\tilde{c}_{1} \in\left[g\left(a_{1}\right), g\left(a_{i}\right)\right], \quad \tilde{c}_{2} \in\left[g\left(b_{1}\right), g\left(b_{j}\right)\right] .
$$

Let

$$
T^{\prime}=\operatorname{conv}\left(\left\{g\left(a_{1}\right), g\left(a_{i}\right), g\left(b_{1}\right), g\left(b_{j}\right)\right\}\right) .
$$

Then $T^{\prime}$ is a convex subset of $\tilde{S}_{1}$, and therefore $T^{\prime}$ is isometric to convex subset of the Euclidean plane. Clearly

$$
\left[g\left(a_{1}\right), g\left(a_{i}\right)\right] \subseteq \tilde{S}_{i} \cap T^{\prime}, \quad\left[g\left(b_{1}\right), g\left(b_{j}\right)\right] \subseteq T^{\prime} \cap \tilde{T}_{j} .
$$

By (9.10), we have $\left\{a_{1}, a_{i}\right\} \cap\left\{b_{1}, b_{j}\right\} \neq \emptyset$. In other words, at least one of the following equalities holds:

$$
a_{1}=b_{1}, \quad a_{1}=b_{j}, \quad a_{i}=b_{1}, \quad a_{i}=b_{j} .
$$

If $a_{1}=b_{1}$, then (9.15), (9.16), (9.17) and Lemma 6.5 imply that

$$
d_{Y}(g(p), g(q)) \geq d_{X}(p, q)
$$

because the subsets $\tilde{S}_{i}, T^{\prime}$ and $\tilde{T}_{j}$ of $Y$ are all isometric to convex subsets of the Euclidean plane, and

$$
\begin{array}{ll}
d_{Y}\left(g\left(a_{1}\right), g(p)\right)=d_{X}\left(a_{1}, p\right), & d_{Y}\left(g(p), g\left(a_{i}\right)\right)=d_{X}\left(p, a_{i}\right), \\
d_{Y}\left(g\left(a_{i}\right), g\left(b_{j}\right)\right)=d_{X}\left(a_{i}, b_{j}\right), & d_{Y}\left(g\left(b_{j}\right), g(q)\right)=d_{X}\left(b_{j}, q\right), \\
d_{Y}\left(g(q), g\left(a_{1}\right)\right)=d_{X}\left(q, a_{1}\right), & d_{Y}\left(g\left(a_{1}\right), g\left(a_{i}\right)\right)=d_{X}\left(a_{1}, a_{i}\right), \\
d_{Y}\left(g\left(a_{1}\right), g\left(b_{j}\right)\right)=d_{X}\left(a_{1}, b_{j}\right) &
\end{array}
$$

by (9.11). If $a_{1}=b_{j}, a_{i}=b_{1}$ or $a_{i}=b_{j}$, then we obtain (9.18) in the same way. Thus (9.18) always holds. By (9.11) and (9.18), $g$ is a map from $\{p, q, x, y, z\}$ to a CAT(0) space with the desired properties. 
Lemma 9.4. Let $\left(X, d_{X}\right)$ be a metric space that satisfies the $\bowtie$-inequalities. Suppose $p, q, x, y, z \in X$ are five distinct points such that $\{p, x, y, z\}$ is under-distance with respect to $\{p, y\}$ or $\{p, z\}$, and $\{q, x, y, z\}$ is underdistance with respect to $\{q, y\}$ or $\{q, z\}$. Then there exist a $\operatorname{CAT}(0)$ space $\left(Y, d_{Y}\right)$ and a map $g:\{x, y, z, p, q\} \rightarrow$ $Y$ such that

$$
d_{Y}(g(p), g(q)) \geq d_{X}(p, q), \quad d_{Y}(g(x), g(a)) \leq d_{X}(x, a), \quad d_{Y}(g(b), g(c))=d_{Y}(b, c)
$$

for any $a, b, c \in\{p, q, y, z\}$ with $\{b, c\} \neq\{p, q\}$.

Proof. Choose $x^{\prime}, y^{\prime}, z^{\prime} \in \mathbb{R}^{2}$ such that

$$
\left\|x^{\prime}-y^{\prime}\right\|=d_{X}(x, y), \quad\left\|y^{\prime}-z^{\prime}\right\|=d_{X}(y, z), \quad\left\|z^{\prime}-x^{\prime}\right\|=d_{X}(z, x) .
$$

Suppose $p^{\prime} \in \mathbb{R}^{2}$ is a point such that

$$
\left\|x^{\prime}-p^{\prime}\right\|=d_{X}(x, p), \quad\left\|p^{\prime}-z^{\prime}\right\|=d_{X}(p, z),
$$

and $p^{\prime}$ is not on the opposite side of $\overleftrightarrow{x^{\prime} z^{\prime}}$ from $y^{\prime}$. Suppose $q_{1}^{\prime} \in \mathbb{R}^{2}$ is a point such that

$$
\left\|x^{\prime}-q_{1}^{\prime}\right\|=d_{X}(x, q), \quad\left\|q_{1}^{\prime}-z^{\prime}\right\|=d_{X}(q, z),
$$

and $q_{1}^{\prime}$ is not on the opposite side of $\overleftrightarrow{x^{\prime} z^{\prime}}$ from $y^{\prime}$. Suppose $q_{2}^{\prime} \in \mathbb{R}^{2}$ is a point such that

$$
\left\|x^{\prime}-q_{2}^{\prime}\right\|=d_{X}(x, q), \quad\left\|q_{2}^{\prime}-y^{\prime}\right\|=d_{X}(q, y),
$$

and $q_{2}^{\prime}$ is not on the opposite side of $\overleftrightarrow{x^{\prime} y^{\prime}}$ from $z^{\prime}$. Such points $p^{\prime}, q_{1}^{\prime}$ and $q_{2}^{\prime}$ are uniquely determined whenever $x^{\prime}, y^{\prime}$ and $z^{\prime}$ are not collinear. We consider four cases.

CASE 1: $\{p, x, y, z\}$ is under-distance with respect to $\{p, y\}$, and $\{q, x, y, z\}$ is under-distance with respect to $\{q, y\}$. According to Corollary 7.6, we divide CASE 1 into the following four subcases.

SubCASE 1A: $p^{\prime} \in \operatorname{conv}\left(\left\{x^{\prime}, y^{\prime}, z^{\prime}\right\}\right)$ and $q_{1}^{\prime} \in \operatorname{conv}\left(\left\{x^{\prime}, y^{\prime}, z^{\prime}\right\}\right)$. In this subcase, $x^{\prime}, y^{\prime}$ and $z^{\prime}$ are not collinear, because otherwise $x^{\prime}, y^{\prime}, z^{\prime}$ and $p^{\prime}$ would be collinear, contradicting Lemma 7.5. Let $T_{1}$ and $T_{2}$ be two isometric copies of $\operatorname{conv}\left(\left\{x^{\prime}, y^{\prime}, z^{\prime}\right\}\right)$. For each $i \in\{1,2\}$ and each $c \in \operatorname{conv}\left(\left\{x^{\prime}, y^{\prime}, z^{\prime}\right\}\right)$, we denote by $\varphi_{i}(c)$ the point in $T_{i}$ corresponding to $c$. Define $\left(T, d_{T}\right)$ to be the piecewise Euclidean simplicial complex constructed from the two simplices $T_{1}$ and $T_{2}$ by identifying $\left[\varphi_{1}\left(x^{\prime}\right), \varphi_{1}\left(y^{\prime}\right)\right]$ with $\left[\varphi_{2}\left(x^{\prime}\right), \varphi_{2}\left(y^{\prime}\right)\right],\left[\varphi_{1}\left(y^{\prime}\right), \varphi_{1}\left(z^{\prime}\right)\right]$ with $\left[\varphi_{2}\left(y^{\prime}\right), \varphi_{2}\left(z^{\prime}\right)\right]$, and $\left[\varphi_{1}\left(z^{\prime}\right), \varphi_{1}\left(x^{\prime}\right)\right]$ with $\left[\varphi_{2}\left(z^{\prime}\right), \varphi_{2}\left(x^{\prime}\right)\right]$. In other words, $T$ is the piecewise Euclidean simplicial complex obtained by gluing $T_{1}$ and $T_{2}$ along their boundaries. As we mentioned in Example 2.5, $T$ is a complete geodesic space with nonnegative Alexandrov curvature, and the natural inclusions of $T_{1}$ and $T_{2}$ into $T$ are both isometric embeddings. In particular, for each $i \in\{1,2\}$, the image $\tilde{T}_{i}$ of $T_{i}$ under the natural inclusion into $T$ is isometric to a convex subset of the Euclidean plane. Define a map $f_{1}:\{p, q, x, y, z\} \rightarrow T$ by sending $x, y, z, p$ and $q$ to the points in $T$ represented by $\varphi_{1}\left(x^{\prime}\right), \varphi_{1}\left(y^{\prime}\right), \varphi_{1}\left(z^{\prime}\right), \varphi_{1}\left(p^{\prime}\right) \in T_{1}$ and $\varphi_{2}\left(q_{1}^{\prime}\right) \in T_{2}$, respectively. Then clearly

$$
d_{T}\left(f_{1}(a), f_{1}(b)\right)=d_{X}(a, b)
$$

for any $a, b \in\{p, q, x, y, z\}$ with $\{a, b\} \notin\{\{p, y\},\{q, y\},\{p, q\}\}$. By the assumption of CASE 1 ,

$$
\begin{aligned}
& d_{T}\left(f_{1}(p), f_{1}(y)\right)=\left\|p^{\prime}-y^{\prime}\right\|>d_{X}(p, y), \\
& d_{T}\left(f_{1}(q), f_{1}(y)\right)=\left\|q_{1}^{\prime}-y^{\prime}\right\|>d_{X}(q, y) .
\end{aligned}
$$

It follows from the definition of $T$ that there exists $c_{0} \in\left[x^{\prime}, y^{\prime}\right] \cup\left[y^{\prime}, z^{\prime}\right] \cup\left[z^{\prime}, x^{\prime}\right]$ such that

$$
d_{T}\left(f_{1}(p), f_{1}(q)\right)=\left\|p^{\prime}-c_{0}\right\|+\left\|c_{0}-q_{1}^{\prime}\right\| .
$$

Hence

$$
d_{T}\left(f_{1}(p), f_{1}(q)\right)=d_{T}\left(f_{1}(p), \tilde{c}_{0}\right)+d_{T}\left(\tilde{c}_{0}, f_{1}(q)\right),
$$

where $\tilde{c}_{0}$ is the point in $T$ represented by $\varphi_{1}\left(c_{0}\right) \in T_{1}$ (or $\varphi_{2}\left(c_{0}\right) \in T_{2}$ ). Let $\Gamma_{0} \subseteq T$ be the image of $\left[\varphi_{1}\left(x^{\prime}\right), \varphi_{1}\left(y^{\prime}\right)\right]$ under the natural inclusion of $T_{1}$ into $T$, which clearly coincides with the image of 
[ $\left.\varphi_{2}\left(x^{\prime}\right), \varphi_{2}\left(y^{\prime}\right)\right]$ under the natural inclusion of $T_{2}$ into $T$. Then it is clear from the definition of $T$ that $\Gamma_{0}$ is a geodesic segment in $T$ with endpoints $f_{1}(x)$ and $f_{1}(y)$, and $\Gamma_{0} \subseteq \tilde{T}_{1} \cap \tilde{T}_{2}$. If $c_{0} \in\left[x^{\prime}, y^{\prime}\right]$, then $\tilde{c}_{0} \in \Gamma_{0}$, and therefore (9.22) and Lemma 3.3 imply that

$$
d_{T}\left(f_{1}(p), f_{1}(q)\right) \geq d_{X}(p, q)
$$

because

$$
\begin{array}{ll}
d_{T}\left(f_{1}(x), f_{1}(p)\right)=d_{X}(x, p), & d_{T}\left(f_{1}(p), f_{1}(y)\right)>d_{X}(p, y), \\
d_{T}\left(f_{1}(y), f_{1}(q)\right)>d_{X}(y, q), & d_{T}\left(f_{1}(q), f_{1}(x)\right)=d_{X}(q, x), \\
d_{T}\left(f_{1}(x), f_{1}(y)\right)=d_{X}(x, y) &
\end{array}
$$

by (9.19), (9.20) and (9.21). If $c_{0} \in\left[y^{\prime}, z^{\prime}\right]$ or $c_{0} \in\left[z^{\prime}, x^{\prime}\right]$, then we obtain (9.23) in the same way. Thus (9.23) always holds in SuBCASE 1A. By (9.19), (9.20), (9.21) and (9.23),

$$
d_{T}\left(f_{1}(x), f_{1}(a)\right)=d_{X}(x, a), \quad d_{T}\left(f_{1}(a), f_{1}(b)\right) \geq d_{X}(a, b) .
$$

for any $a, b \in\{p, q, y, z\}$. Therefore, Lemma 9.1 implies that there exist a CAT(0) space $\left(Y_{1}, d_{Y_{1}}\right)$ and a map $g_{1}:\{p, q, x, y, z\} \rightarrow Y_{1}$ such that

$$
d_{Y_{1}}\left(g_{1}(x), g_{1}(a)\right) \leq d_{X}(x, a), \quad d_{Y_{1}}\left(g_{1}(a), g_{1}(b)\right)=d_{X}(a, b)
$$

for any $a, b \in\{p, q, y, z\}$. Thus $g_{1}$ is a map from $\{p, q, x, y, z\}$ to a CAT(0) space with the desired properties.

SubCASE 1B: $p^{\prime} \in \operatorname{conv}\left(\left\{x^{\prime}, y^{\prime}, z^{\prime}\right\}\right)$ and $y^{\prime} \in \operatorname{conv}\left(\left\{q_{1}^{\prime}, x^{\prime}, z^{\prime}\right\}\right)$. In this subcase, we define a map $f_{2}:$ $\{p, q, x, y, z\} \rightarrow \mathbb{R}^{2}$ by

$$
f_{2}(x)=x^{\prime}, \quad f_{2}(y)=y^{\prime}, \quad f_{2}(z)=z^{\prime}, \quad f_{2}(p)=p^{\prime}, \quad f_{2}(q)=q_{1}^{\prime} .
$$

Then

$$
\left\|f_{2}(a)-f_{2}(b)\right\|=d_{X}(a, b)
$$

for any $a, b \in\{p, q, x, y, z\}$ with $\{a, b\} \notin\{\{p, y\},\{q, y\},\{p, q\}\}$. By the assumption of CASE 1 ,

$$
\begin{aligned}
& \left\|f_{2}(p)-f_{2}(y)\right\|=\left\|p^{\prime}-y^{\prime}\right\|>d_{X}(p, y), \\
& \left\|f_{2}(q)-f_{2}(y)\right\|=\left\|q_{1}^{\prime}-y^{\prime}\right\|>d_{X}(q, y) .
\end{aligned}
$$

It follows from the assumption of SUBCASE 1B that the line segment $\left[f_{2}(p), f_{2}(q)\right]$ has a nonempty intersection with $\left[f_{2}(x), f_{2}(y)\right]$ or $\left[f_{2}(y), f_{2}(z)\right]$. If $\left[f_{2}(p), f_{2}(q)\right]$ has a nonempty intersection with $\left[f_{2}(x), f_{2}(y)\right]$, then Lemma 3.2 implies that

$$
\left\|f_{2}(p)-f_{2}(q)\right\| \geq d_{X}(p, q)
$$

because

$$
\begin{array}{ll}
\left\|f_{2}(x)-f_{2}(p)\right\|=d_{X}(x, p), & \left\|f_{2}(p)-f_{2}(y)\right\|>d_{X}(p, y), \\
\left\|f_{2}(y)-f_{2}(q)\right\|>d_{X}(y, q), & \left\|f_{2}(q)-f_{2}(x)\right\|=d_{X}(q, x), \\
\left\|f_{2}(x)-f_{2}(y)\right\|=d_{X}(x, y) &
\end{array}
$$

by (9.24), (9.25) and (9.26). If $\left[f_{2}(p), f_{2}(q)\right]$ has a nonempty intersection with $\left[f_{2}(y), f_{2}(z)\right]$, then we obtain $(9.27)$ in the same way. Thus (9.27) always holds in SUBCASE 1B. By (9.24), (9.25), (9.26) and (9.27),

$$
\left\|f_{2}(x)-f_{2}(a)\right\|=d_{X}(x, a), \quad\left\|f_{2}(a)-f_{2}(b)\right\| \geq d_{X}(a, b)
$$

for any $a, b \in\{p, q, y, z\}$. Therefore, because $\mathbb{R}^{2}$ is a complete geodesic space with nonnegative Alexandrov curvature, Lemma 9.1 implies that there exist a $\operatorname{CAT}(0)$ space $\left(Y_{2}, d_{Y_{2}}\right)$ and a map $g_{2}:\{p, q, x, y, z\} \rightarrow Y_{2}$ such that

$$
d_{Y_{2}}\left(g_{2}(x), g_{2}(a)\right) \leq d_{X}(x, a), \quad d_{Y_{2}}\left(g_{2}(a), g_{2}(b)\right)=d_{X}(a, b)
$$


for any $a, b \in\{p, q, y, z\}$. Thus $g_{2}$ is a map from $\{p, q, x, y, z\}$ to a CAT(0) space with the desired properties.

SUBCASE 1C: $y^{\prime} \in \operatorname{conv}\left(\left\{p^{\prime}, x^{\prime}, z^{\prime}\right\}\right)$ and $q_{1}^{\prime} \in \operatorname{conv}\left(\left\{x^{\prime}, y^{\prime}, z^{\prime}\right\}\right)$. In this subcase, the existence of a map from $\{p, q, x, y, z\}$ to a $\operatorname{CAT}(0)$ space with the desired properties is proved in exactly the same way as in SUBCASE 1B.

SUBCASE 1D: $y^{\prime} \in \operatorname{conv}\left(\left\{p^{\prime}, x^{\prime}, z^{\prime}\right\}\right)$ and $y^{\prime} \in \operatorname{conv}\left(\left\{q_{1}^{\prime}, x^{\prime}, z^{\prime}\right\}\right)$. In this subcase, Corollary 7.6 implies that

$$
p^{\prime} \notin \operatorname{conv}\left(\left\{x^{\prime}, y^{\prime}, z^{\prime}\right\}\right) \text {. }
$$

It follows that $\{p, x, y, z\}$ is not under-distance with respect to $\{p, x\}$, because otherwise Lemma 7.8 and the assumption that $\{p, x, y, z, w\}$ is under-distance with respect to $\{p, y\}$ would imply that $p^{\prime} \in$ $\operatorname{conv}\left(\left\{x^{\prime}, y^{\prime}, z^{\prime}\right\}\right)$, contradicting (9.28). Hence $\{p, x, y, z\}$ is over-distance with respect to $\{p, x\}$ by Proposition 7.2. Similarly, $\{q, x, y, z\}$ is over-distance with respect to $\{q, x\}$. Therefore, Lemma 9.3 implies that there exist a $\operatorname{CAT}(0)$ space $\left(Y_{3}, d_{Y_{3}}\right)$ and a map $g_{3}:\{p, q, x, y, z\} \rightarrow Y_{3}$ such that

$$
d_{Y_{3}}\left(g_{3}(p), g_{3}(q)\right) \geq d_{X}(p, q), \quad d_{Y_{3}}\left(g_{3}(a), g_{3}(b)\right)=d_{X}(a, b)
$$

for any $a, b \in\{p, q, x, y, z\}$ with $\{a, b\} \neq\{p, q\}$. Thus $g_{3}$ is a map from $\{p, q, x, y, z\}$ to a CAT(0) space with the desired properties.

By Corollary 7.6, the above four subcases exhaust all possibilities in CASE 1.

CASE 2: $\{p, x, y, z\}$ is under-distance with respect to $\{p, y\}$ and $\{q, x, y, z\}$ is under-distance with respect to $\{q, z\}$. According to Corollary 7.6, we divide CASE 2 into the following four subcases.

SubCASE 2A: $p^{\prime} \in \operatorname{conv}\left(\left\{x^{\prime}, y^{\prime}, z^{\prime}\right\}\right)$ and $q_{2}^{\prime} \in \operatorname{conv}\left(\left\{x^{\prime}, y^{\prime}, z^{\prime}\right\}\right)$. In this subcase, $x^{\prime}, y^{\prime}$ and $z^{\prime}$ are not collinear, because otherwise $x^{\prime}, y^{\prime}, z^{\prime}$ and $p^{\prime}$ would be collinear, contradicting Lemma 7.5. Let $T_{1}, T_{2}, \varphi_{1}$, $\varphi_{2}$ and $\left(T, d_{T}\right)$ be as in SubcaSe 1A. Define a map $f_{4}:\{p, q, x, y, z\} \rightarrow T$ by sending $x, y, z, p$ and $q$ to the points in $T$ represented by $\varphi_{1}\left(x^{\prime}\right), \varphi_{1}\left(y^{\prime}\right), \varphi_{1}\left(z^{\prime}\right), \varphi_{1}\left(p^{\prime}\right) \in T_{1}$ and $\varphi_{2}\left(q_{2}^{\prime}\right) \in T_{2}$, respectively. Then a similar argument as in SUBCASE 1A yields

$$
d_{T}\left(f_{4}(x), f_{4}(a)\right)=d_{X}(x, a), \quad d_{T}\left(f_{4}(a), f_{4}(b)\right) \geq d_{X}(a, b)
$$

for any $a, b \in\{p, q, y, z\}$. Therefore, because $T$ is a complete geodesic space with nonnegative Alexandrov curvature, Lemma 9.1 implies that there exist a $\operatorname{CAT}(0)$ space $\left(Y_{4}, d_{Y_{4}}\right)$ and a map $g_{4}:\{p, q, x, y, z\} \rightarrow Y_{4}$ such that

$$
d_{Y_{4}}\left(g_{4}(x), g_{4}(a)\right) \leq d_{X}(x, a), \quad d_{Y_{4}}\left(g_{4}(a), g_{4}(b)\right)=d_{X}(a, b)
$$

for any $a, b \in\{p, q, y, z\}$. Thus $g_{4}$ is a map from $\{p, q, x, y, z\}$ to a CAT(0) space with the desired properties.

SUBCASE 2B: $p^{\prime} \in \operatorname{conv}\left(\left\{x^{\prime}, y^{\prime}, z^{\prime}\right\}\right)$ and $z^{\prime} \in \operatorname{conv}\left(\left\{q_{2}^{\prime}, x^{\prime}, y^{\prime}\right\}\right)$. In this subcase, we define a map $f_{5}$ : $\{p, q, x, y, z\} \rightarrow \mathbb{R}^{2}$ by

$$
f_{5}(x)=x^{\prime}, \quad f_{5}(y)=y^{\prime}, \quad f_{5}(z)=z^{\prime}, \quad f_{5}(p)=p^{\prime}, \quad f_{5}(q)=q_{2}^{\prime} .
$$

Then a similar argument as in SUBCASE 1B implies that

$$
\left\|f_{5}(x)-f_{5}(a)\right\|=d_{X}(x, a), \quad\left\|f_{5}(a)-f_{5}(b)\right\| \geq d_{X}(a, b)
$$

for any $a, b \in\{p, q, y, z\}$. Therefore, because $\mathbb{R}^{2}$ is a complete geodesic space with nonnegative Alexandrov curvature, Lemma 9.1 implies that there exist a $\operatorname{CAT}(0)$ space $\left(Y_{5}, d_{Y_{5}}\right)$ and a map $g_{5}:\{p, q, x, y, z\} \rightarrow Y_{5}$ such that

$$
d_{Y_{5}}\left(g_{5}(x), g_{5}(a)\right) \leq d_{X}(x, a), \quad d_{Y_{5}}\left(g_{5}(a), g_{5}(b)\right)=d_{X}(a, b)
$$

for any $a, b \in\{p, q, y, z\}$. Thus $g_{5}$ is a map from $\{p, q, x, y, z\}$ to a CAT(0) space with the desired properties.

SUBCASE 2C: $y^{\prime} \in \operatorname{conv}\left(\left\{x^{\prime}, z^{\prime}, p^{\prime}\right\}\right)$ and $q_{2}^{\prime} \in \operatorname{conv}\left(\left\{x^{\prime}, y^{\prime}, z^{\prime}\right\}\right)$. In this subcase, the existence of a map from $\{p, q, x, y, z\}$ to a $\operatorname{CAT}(0)$ space with the desired properties is proved in exactly the same way as in SUBCASE 2B. 
SubCASE 2D: $y^{\prime} \in \operatorname{conv}\left(\left\{p^{\prime}, x^{\prime}, z^{\prime}\right\}\right)$ and $z^{\prime} \in \operatorname{conv}\left(\left\{q_{2}^{\prime}, x^{\prime}, y^{\prime}\right\}\right)$. In this subcase, it follows from the same argument as in SUBCASE 1D that $\{p, x, y, z\}$ is over-distance with respect to $\{p, x\}$, and $\{q, x, y, z\}$ is overdistance with respect to $\{q, x\}$. Therefore, Lemma 9.3 implies that there exist a CAT(0) space $\left(Y_{6}, d_{Y_{6}}\right)$ and a map $g_{6}:\{p, q, x, y, z\} \rightarrow Y_{6}$ such that

$$
d_{Y_{6}}\left(g_{6}(p), g_{6}(q)\right) \geq d_{X}(p, q), \quad d_{Y_{6}}\left(g_{6}(a), g_{6}(b)\right)=d_{X}(a, b)
$$

for any $a, b \in\{p, q, x, y, z\}$ with $\{a, b\} \neq\{p, q\}$. Thus $g_{6}$ is a map from $\{p, q, x, y, z\}$ to a CAT(0) space with the desired properties.

By Corollary 7.6, the above four subcases exhaust all possibilities in CASE 2.

CASE 3: $\{p, x, y, z\}$ is under-distance with respect to $\{p, z\}$, and $\{q, x, y, z\}$ is under-distance with respect to $\{q, y\}$. In this case, the existence of a map from $\{p, q, x, y, z\}$ to a CAT(0) space with the desired properties is proved in exactly the same way as in CASE 2.

CASE 4: $\{p, x, y, z\}$ is under-distance with respect to $\{p, z\}$, and $\{q, x, y, z\}$ is under-distance with respect to $\{q, z\}$. In this case, the existence of a map from $\{p, q, x, y, z\}$ to a CAT(0) space with the desired properties is proved in exactly the same way as in CASE 1.

CASE 1, CASE 2, CASE 3 and CASE 4 exhaust all possibilities.

Lemma 9.5. Let $\left(X, d_{X}\right)$ be a metric space that satisfies the $\bigotimes$-inequalities. Suppose $p, q, x, y, z \in X$ are five distinct points such that $\{p, x, y, z\}$ admits an isometric embedding into $\mathbb{R}^{3}$, and $\{q, x, y, z\}$ does not admit an isometric embedding into $\mathbb{R}^{3}$. Then there exist a CAT( 0$)$ space $\left(Y, d_{Y}\right)$ and a map $g:\{p, q, x, y, z\} \rightarrow Y$ such that

$$
d_{Y}(g(p), g(q)) \geq d_{X}(p, q), \quad d_{Y}(g(x), g(a)) \leq d_{X}(x, a), \quad d_{Y}(g(b), g(c))=d_{Y}(b, c)
$$

for any $a, b, c \in\{p, q, y, z\}$ with $\{b, c\} \neq\{p, q\}$.

Proof. Let $\varphi_{0}:\{p, x, y, z\} \rightarrow \mathbb{R}^{3}$ be an isometric embedding, and let

$$
T_{0}=\operatorname{conv}\left(\left\{\varphi_{0}(x), \varphi_{0}(y), \varphi_{0}(z)\right\}\right) .
$$

We consider two cases.

CASE 1: $\{q, x, y, z\}$ is over-distance with respect to $\{q, y\}$ or $\{q, z\}$. In this case, we may assume without loss of generality that $\{q, x, y, z\}$ is over-distance with respect to $\{q, y\}$. Then Lemma 7.7 implies that $\pi<$ $\tilde{Z} y x z+\tilde{Z} z x q$ or $\pi<\tilde{Z} y z x+\tilde{Z} x z q$. We may assume further without loss of generality that the former inequality holds. Let $Y_{1}^{\prime}=D(x ; y, z, q)$, and let $\varphi_{1}:\{x, y, z, q\} \rightarrow Y_{1}^{\prime}$ be the natural inclusion. Then $Y_{1}^{\prime}$ is a CAT(0) space, and $\varphi_{1}$ is an isometric embedding by Lemma 7.14. We set

$$
T_{1}=T_{Y_{1}^{\prime}}(x, y, z), \quad T_{2}=T_{Y_{1}^{\prime}}(x, z, q), \quad T_{2}=T_{Y_{1}^{\prime}}(x, q, y)
$$

By Lemma 7.14, $T_{1}, T_{2}$ and $T_{3}$ are closed convex subsets of $Y_{1}^{\prime}$, all of which are isometric to convex subsets of the Euclidean plane. It also follows from Lemma 7.14 that there exists an isometry $h_{1}: T_{1} \rightarrow T_{0}$ such that

$$
h_{1}\left(\varphi_{1}(x)\right)=\varphi_{0}(x), \quad h_{1}\left(\varphi_{1}(y)\right)=\varphi_{0}(y), \quad h_{1}\left(\varphi_{1}(z)\right)=\varphi_{0}(z) .
$$

Define a metric space $\left(Y_{1}, d_{Y_{1}}\right)$ to be the gluing of $Y_{1}^{\prime}$ and $\mathbb{R}^{3}$ along $h_{1}$. Then $Y_{1}$ is a CAT(0) space by Reshetnyak's gluing theorem, and the natural inclusions of $Y_{1}^{\prime}$ and $\mathbb{R}^{3}$ into $Y_{1}$ are both isometric embeddings. In particular, for each $i \in\{1,2,3\}$, the image $\tilde{T}_{i}$ of $T_{i}$ under the natural inclusion of $Y_{1}^{\prime}$ into $Y_{1}$ is isometric to a convex subset of the Euclidean plane. Define a map $g_{1}:\{p, q, x, y, z\} \rightarrow Y_{1}$ by sending $x, y, z, p$ and $q$ to the points in $Y_{1}$ represented by $\varphi_{0}(x), \varphi_{0}(y), \varphi_{0}(z), \varphi_{0}(p) \in \mathbb{R}^{3}$ and $\varphi_{1}(q) \in Y_{1}^{\prime}$, respectively. Then

$$
\begin{aligned}
& d_{Y_{1}}\left(g_{1}(q), g_{1}(a)\right)=d_{Y_{1}^{\prime}}\left(\varphi_{1}(q), \varphi_{1}(a)\right)=d_{X}(q, a) \\
& d_{Y_{1}}\left(g_{1}(b), g_{1}(c)\right)=\left\|\varphi_{0}(b)-\varphi_{0}(c)\right\|=d_{X}(b, c),
\end{aligned}
$$

for any $a \in\{x, y, z\}$ and any $b, c \in\{p, x, y, z\}$. It follows from the definition of $Y_{1}$ that there exists $r_{0} \in T_{1}$ such that

$$
d_{Y_{1}}\left(g_{1}(p), g_{1}(q)\right)=\left\|\varphi_{0}(p)-h_{1}\left(r_{0}\right)\right\|+d_{Y_{1}^{\prime}}\left(r_{0}, \varphi_{1}(q)\right) .
$$


It is clear from the definition of $Y_{1}^{\prime}=D(x ; y, z, q)$ that there exists

$$
r_{1} \in\left[\varphi_{1}(x), \varphi_{1}(y)\right] \cup\left[\varphi_{1}(x), \varphi_{1}(z)\right]
$$

such that

$$
d_{Y_{1}^{\prime}}\left(r_{0}, \varphi_{1}(q)\right)=d_{Y_{1}^{\prime}}\left(r_{0}, r_{1}\right)+d_{Y_{1}^{\prime}}\left(r_{1}, \varphi_{1}(q)\right) .
$$

By (9.31), (9.32) and the triangle inequality for $Y_{1}$,

$$
\begin{aligned}
d_{Y_{1}}\left(g_{1}(p), g_{1}(q)\right) & =\left\|\varphi_{0}(p)-h_{1}\left(r_{0}\right)\right\|+d_{Y_{1}^{\prime}}\left(r_{0}, r_{1}\right)+d_{Y_{1}^{\prime}}\left(r_{1}, \varphi_{1}(q)\right) \\
& =d_{Y_{1}}\left(g_{1}(p), \tilde{r}_{0}\right)+d_{Y_{1}}\left(\tilde{r}_{0}, \tilde{r}_{1}\right)+d_{Y_{1}}\left(\tilde{r}_{1}, g_{1}(q)\right) \\
& =d_{Y_{1}}\left(g_{1}(p), \tilde{r}_{1}\right)+d_{Y_{1}}\left(\tilde{r}_{1}, g_{1}(q)\right),
\end{aligned}
$$

where $\tilde{r}_{0}$ and $\tilde{r}_{1}$ are the points in $Y_{1}$ represented by $r_{0} \in Y_{1}^{\prime}$ and $r_{1} \in Y_{1}^{\prime}$, respectively. Let $\tilde{R}$ be the image of $\mathbb{R}^{3}$ under the natural inclusion of $\mathbb{R}^{3}$ into $Y_{1}$. If $r_{1} \in\left[\varphi_{1}(x), \varphi_{1}(y)\right]$, then $\tilde{r}_{1}$ lies on the geodesic segment $\left[g_{1}(x), g_{1}(y)\right]$ in $Y_{1}$ clearly, and therefore (9.29), (9.30), (9.33) and Lemma 3.3 imply that

$$
d_{Y_{1}}\left(g_{1}(p), g_{1}(q)\right) \geq d_{X}(p, q)
$$

because $\left[g_{1}(x), g_{1}(y)\right] \subseteq \tilde{R} \cap \tilde{T}_{2}$. If $r_{1} \in\left[\varphi_{1}(x), \varphi_{1}(z)\right]$, then we obtain (9.34) in the same way. Thus (9.34) always holds in CASE 1. By (9.29), (9.30), and (9.34), $g_{1}$ is a map from $\{p, q, x, y, z\}$ to a CAT(0) space with the desired properties.

CASE 2: $\{q, x, y, z\}$ is under-distance with respect to $\{q, y\}$ and $\{q, z\}$. In this case, we choose $q^{\prime}, x^{\prime}, y^{\prime}, z^{\prime} \in$ $\mathbb{R}^{2}$ such that

$$
\begin{aligned}
& \left\|x^{\prime}-y^{\prime}\right\|=d_{X}(x, y), \quad\left\|y^{\prime}-z^{\prime}\right\|=d_{X}(y, z), \quad\left\|z^{\prime}-x^{\prime}\right\|=d_{X}(z, x), \\
& \left\|x^{\prime}-q^{\prime}\right\|=d_{X}(x, q), \quad\left\|q^{\prime}-z^{\prime}\right\|=d_{X}(q, z),
\end{aligned}
$$

and $q^{\prime}$ is not on the opposite side of $\overleftrightarrow{x^{\prime} z^{\prime}}$ from $y^{\prime}$. Then Lemma 7.8, Corollary 7.9 and the assumption of CASE 2 imply that $q^{\prime} \in \operatorname{conv}\left(\left\{x^{\prime}, y^{\prime}, z^{\prime}\right\}\right)$, and that $x^{\prime}, y^{\prime}$ and $z^{\prime}$ are not collinear. Set $T_{4}=\operatorname{conv}\left(\left\{x^{\prime}, y^{\prime}, z^{\prime}\right\}\right)$. Then there exists an isometry $h_{2}: T_{4} \rightarrow T_{0}$ such that

$$
h_{2}\left(x^{\prime}\right)=\varphi_{0}(x), \quad h_{2}\left(y^{\prime}\right)=\varphi_{0}(y), \quad h_{2}\left(z^{\prime}\right)=\varphi_{0}(z) .
$$

We divide CASE 2 into three subcases.

SUBCASE 2A: $\varphi_{0}(p), \varphi_{0}(x), \varphi_{0}(y)$ and $\varphi_{0}(z)$ are not coplanar. Let $S$ be the boundary of $\operatorname{conv}\left(\left\{\varphi_{0}(p), \varphi_{0}(x), \varphi_{0}(y), \varphi_{0}(z)\right\}\right)$ in $\mathbb{R}^{3}$ equipped with the induced length metric $d_{S}$. As we mentioned in Example 2.4, $\left(S, d_{S}\right)$ is a complete geodesic space with nonnegative Alexandrov curvature. Clearly $S$ is the union of four subsets $\operatorname{conv}\left(\left\{\varphi_{0}(p), \varphi_{0}(x), \varphi_{0}(y)\right\}\right), \operatorname{conv}\left(\left\{\varphi_{0}(p), \varphi_{0}(y), \varphi_{0}(z)\right\}\right), \operatorname{conv}\left(\left\{\varphi_{0}(p), \varphi_{0}(z), \varphi_{0}(x)\right\}\right)$ and $T_{0}$ of $\mathbb{R}^{3}$. On each of these four subsets, $d_{S}$ coincides with the Euclidean metric on $\mathbb{R}^{3}$. In particular, these four subsets are all isometric to convex subsets of the Euclidean plane even as subsets of $\left(S, d_{S}\right)$. Define a map $f_{2}:\{p, q, x, y, z\} \rightarrow S$ by sending each $a \in\{p, x, y, z\}$ to $\varphi_{0}(a)$, and $q$ to $h_{2}\left(q^{\prime}\right)$. Then

$$
\begin{aligned}
& d_{S}\left(f_{2}(q), f_{2}(a)\right)=\left\|q^{\prime}-a^{\prime}\right\|=d_{X}(q, a) \\
& d_{S}\left(f_{2}(b), f_{2}(c)\right)=\left\|\varphi_{0}(b)-\varphi_{0}(c)\right\|=d_{X}(b, c)
\end{aligned}
$$

for any $a \in\{x, z\}$ and $b, c \in\{p, x, y, z\}$. By the assumption of CASE 2,

$$
d_{S}\left(f_{2}(q), f_{2}(y)\right)=\left\|q^{\prime}-y^{\prime}\right\|>d_{X}(q, y) .
$$

Fix a geodesic segment $\Gamma_{0}$ in $\left(S, d_{S}\right)$ with endpoints $f_{2}(p)$ and $f_{2}(q)$. Clearly $\Gamma_{0}$ has a nonempty intersection with the line segment $\left[\varphi_{0}(x), \varphi_{0}(y)\right]$, $\left[\varphi_{0}(y), \varphi_{0}(z)\right]$ or $\left[\varphi_{0}(z), \varphi_{0}(x)\right]$. If $\Gamma_{0}$ has a nonempty intersection with $\left[\varphi_{0}(x), \varphi_{0}(y)\right]$, then Lemma 3.3 implies that

$$
d_{S}\left(f_{2}(p), f_{2}(q)\right) \geq d_{X}(p, q)
$$


because $\left[\varphi_{0}(x), \varphi_{0}(y)\right]=\operatorname{conv}\left(\left\{\varphi_{0}(p), \varphi_{0}(x), \varphi_{0}(y)\right\}\right) \cap T_{0}$ is a geodesic segment even in $\left(S, d_{S}\right)$ with endpoints $f_{2}(x)$ and $f_{2}(y)$, and

$$
\begin{array}{ll}
d_{S}\left(f_{2}(x), f_{2}(p)\right)=d_{X}(x, p), & d_{S}\left(f_{2}(p), f_{2}(y)\right)=d_{X}(p, y), \\
d_{S}\left(f_{2}(y), f_{2}(q)\right)>d_{X}(y, q), & d_{S}\left(f_{2}(q), f_{2}(x)\right)=d_{X}(q, x), \\
d_{S}\left(f_{2}(x), f_{2}(y)\right)=d_{X}(x, y) &
\end{array}
$$

by (9.35), (9.36) and (9.37). If $\Gamma_{0}$ has a nonempty intersection with $\left[\varphi_{0}(y), \varphi_{0}(z)\right]$ or $\left[\varphi_{0}(z), \varphi_{0}(x)\right]$, then we obtain (9.38) in the same way. Thus (9.38) always holds in SuBCASE 2A. By (9.35), (9.36), (9.37) and (9.38), $f_{2}$ satisfies

$$
d_{S}\left(f_{2}(x), f_{2}(a)\right)=d_{X}(x, a), \quad d_{S}\left(f_{2}(a), f_{2}(b)\right) \geq d_{X}(a, b)
$$

for any $a, b \in\{p, q, y, z\}$. Therefore, Lemma 9.1 implies that there exist a CAT(0) space $\left(Y_{2}, d_{Y_{2}}\right)$ and a map $g_{2}:\{p, q, x, y, z\} \rightarrow Y_{2}$ such that

$$
d_{Y_{2}}\left(g_{2}(x), g_{2}(a)\right) \leq d_{X}(x, a), \quad d_{Y_{2}}\left(g_{2}(a), g_{2}(b)\right)=d_{X}(a, b)
$$

for any $a, b \in\{p, q, y, z\}$. Thus $g_{2}$ is a map from $\{p, q, x, y, z\}$ to a CAT(0) space with the desired properties.

SUBCASE 2B: $\varphi_{0}(p), \varphi_{0}(x), \varphi_{0}(y)$ and $\varphi_{0}(z)$ are coplanar, and $\varphi_{0}(p) \in T_{0}$. In this case, we define $\left(T, d_{T}\right)$ to be the piecewise Euclidean simplicial complex constructed from two simplices $T_{0}$ and $T_{4}$ by identifying $\left[\varphi_{0}(x), \varphi_{0}(y)\right]$ with $\left[x^{\prime}, y^{\prime}\right],\left[\varphi_{0}(y), \varphi_{0}(z)\right]$ with $\left[y^{\prime}, z^{\prime}\right]$, and $\left[\varphi_{0}(z), \varphi_{0}(x)\right]$ with $\left[z^{\prime}, x^{\prime}\right]$. In other words, $T$ is the piecewise Euclidean simplicial complex obtained by gluing $T_{0}$ and $T_{4}$ along their boundaries. As we mentioned in Example 2.5, $T$ is a complete geodesic space with nonnegative Alexandrov curvature, and the natural inclusions of $T_{0}$ and $T_{4}$ into $T$ are both isometric embeddings. Define a map $f_{3}:\{p, q, x, y, z\} \rightarrow T$ by sending $x, y, z, p$ and $q$ to the points in $T$ represented by $\varphi_{0}(x), \varphi_{0}(y), \varphi_{0}(z), \varphi_{0}(p) \in T_{0}$ and $q^{\prime} \in T_{4}$, respectively. Then a similar argument as in SUBCASE 1A in the proof of Lemma 9.4 yields

$$
d_{T}\left(f_{3}(x), f_{3}(a)\right)=d_{X}(x, a), \quad d_{T}\left(f_{3}(a), f_{3}(b)\right) \geq d_{X}(a, b)
$$

for any $a, b \in\{p, q, y, z\}$. Therefore, Lemma 9.1 implies that there exist a $\operatorname{CAT}(0)$ space $\left(Y_{3}, d_{Y_{3}}\right)$ and a map $g_{3}:\{p, q, x, y, z\} \rightarrow Y_{3}$ such that

$$
d_{Y_{3}}\left(g_{3}(x), g_{3}(a)\right) \leq d_{X}(x, a), \quad d_{Y_{3}}\left(g_{3}(a), g_{3}(b)\right)=d_{X}(a, b)
$$

for any $a, b \in\{p, q, y, z\}$. Thus $g_{3}$ is a map from $\{p, q, x, y, z\}$ to a CAT(0) space with the desired properties.

SUBCASE 2C: $\varphi_{0}(p), \varphi_{0}(x), \varphi_{0}(y)$ and $\varphi_{0}(z)$ are coplanar, and $\varphi_{0}(p) \notin T_{0}$. Let $\alpha$ be the plane in $\mathbb{R}^{3}$ through $\varphi_{0}(x), \varphi_{0}(y), \varphi_{0}(z)$ and $\varphi_{0}(p)$. Define a map $f_{4}:\{p, q, x, y, z\} \rightarrow \alpha$ by sending each $a \in\{p, x, y, z\}$ to $\varphi_{0}(a)$, and $q$ to $h_{2}\left(q^{\prime}\right)$. Then

$$
\begin{aligned}
\left\|f_{4}(q)-f_{4}(a)\right\| & =\left\|q^{\prime}-a^{\prime}\right\|=d_{X}(q, a) \\
\left.\| f_{4}(b)-f_{4}(c)\right) \| & =\left\|\varphi_{0}(b)-\varphi_{0}(c)\right\|=d_{X}(b, c)
\end{aligned}
$$

for any $a \in\{x, z\}$ and any $b, c \in\{p, x, y, z\}$. By the assumption of CASE 2,

$$
\left\|f_{4}(q)-f_{4}(y)\right\|=\left\|q^{\prime}-y^{\prime}\right\|>d_{X}(q, y) .
$$

Because $f_{4}(q) \in T_{0}$ and $f_{4}(p) \in \alpha \backslash T_{0},\left[f_{4}(p), f_{4}(q)\right]$ has a nonempty intersection with $\left[f_{4}(x), f_{4}(y)\right]$, $\left[f_{4}(y), f_{4}(z)\right]$ or $\left[f_{4}(z), f_{4}(x)\right]$. If $\left[f_{4}(p), f_{4}(q)\right]$ has a nonempty intersection with $\left[f_{4}(x), f_{4}(y)\right]$, then Lemma 3.2 implies that

$$
\left\|f_{4}(p)-f_{4}(q)\right\| \geq d_{X}(p, q)
$$

because

$$
\begin{array}{ll}
\left\|f_{4}(x)-f_{4}(p)\right\|=d_{X}(x, p), & \left\|f_{4}(p)-f_{4}(y)\right\|=d_{X}(p, y), \\
\left\|f_{4}(y)-f_{4}(q)\right\|>d_{X}(y, q), & \left\|f_{4}(q)-f_{4}(x)\right\|=d_{X}(q, x), \\
\left\|f_{4}(x)-f_{4}(y)\right\| & =d_{X}(x, y)
\end{array}
$$


by (9.39), (9.40) and (9.41). If $\left[f_{4}(p), f_{4}(q)\right]$ has a nonempty intersection with $\left[f_{4}(y), f_{4}(z)\right]$ or $\left[f_{4}(z), f_{4}(x)\right]$, then we obtain (9.42) in the same way. Thus (9.42) always holds in SuBCASE 2c. By (9.39), (9.40), (9.41) and (9.42),

$$
\left\|f_{4}(x)-f_{4}(a)\right\|=d_{X}(x, a), \quad\left\|f_{4}(a)-f_{4}(b)\right\| \geq d_{X}(a, b)
$$

for any $a, b \in\{p, q, y, z\}$. Therefore, because $\alpha$ is a complete geodesic space with nonnegative Alexandrov curvature, Lemma 9.1 implies that there exist a $\operatorname{CAT}(0)$ space $\left(Y_{4}, d_{Y_{4}}\right)$ and a map $g_{4}:\{p, q, x, y, z\} \rightarrow Y_{4}$ such that

$$
d_{Y_{4}}\left(g_{4}(x), g_{4}(a)\right) \leq d_{X}(x, a), \quad d_{Y_{4}}\left(g_{4}(a), g_{4}(b)\right)=d_{X}(a, b)
$$

for any $a, b \in\{p, q, y, z\}$. Thus $g_{4}$ is a map from $\{p, q, x, y, z\}$ to a CAT(0) space with the desired properties.

The above three subcases clearly exhaust all possibilities in CASE 2. By Proposition 7.2, CASE 1 and CASE 2 exhaust all possibilities.

Using the facts that we have proved so far, we now prove the following proposition.

Proposition 9.6. If a metric space $X$ satisfies the $\otimes$-inequalities, then $X$ satisfies the $G_{9}^{(5)}(0)$ condition.

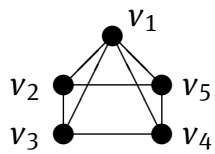

Figure 9.1

Proof. Let $\left(X, d_{X}\right)$ be a metric space that satisfies the $\otimes$-inequalities. Let $V$ and $E$ be the vertex set and the edge set of $G_{9}^{(5)}(0)$, respectively. We set

$$
\begin{aligned}
& V=\left\{v_{1}, v_{2}, v_{3}, v_{4}, v_{5}\right\}, \\
& E=\left\{\left\{v_{1}, v_{2}\right\},\left\{v_{1}, v_{3}\right\},\left\{v_{1}, v_{4}\right\},\left\{v_{1}, v_{5}\right\},\left\{v_{2}, v_{3}\right\},\left\{v_{3}, v_{4}\right\},\left\{v_{4}, v_{5}\right\},\left\{v_{5}, v_{2}\right\}\right\},
\end{aligned}
$$

as shown in FIGURE 9.1. Fix a map $f: V \rightarrow X$, and set

$$
x_{i}=f\left(v_{i}\right), \quad d_{i j}=d_{X}\left(f\left(v_{i}\right), f\left(v_{j}\right)\right)
$$

for any $i, j \in\{1,2,3,4,5\}$. By Theorem 1.7, if $d_{i j}=0$ for some $i, j \in\{1,2,3,4,5\}$ with $i \neq j$, then there exist a CAT(0) space $\left(Y_{0}, d_{Y_{0}}\right)$ and a map $g: V \rightarrow Y_{0}$ such that $d_{Y_{0}}\left(g_{0}\left(v_{i}\right), g_{0}\left(v_{j}\right)\right)=d_{i j}$ for any $i, j \in\{1,2,3,4,5\}$. Therefore, we assume that $d_{i j}>0$ for any $i, j \in\{1,2,3,4,5\}$ with $i \neq j$. We define $V^{\prime}, V_{1}^{\prime}, V_{2}^{\prime} \subseteq X$ by

$$
V^{\prime}=\left\{x_{1}, x_{2}, x_{3}, x_{4}, x_{5}\right\}, \quad V_{1}^{\prime}=\left\{x_{2}, x_{1}, x_{3}, x_{5}\right\}, \quad V_{2}^{\prime}=\left\{x_{4}, x_{1}, x_{3}, x_{5}\right\} .
$$

We consider three cases.

CASE 1: Both $V_{1}^{\prime}$ and $V_{2}^{\prime}$ admit isometric embeddings into $\mathbb{R}^{3}$. In this case, Lemma 9.2 implies that there exist a $\operatorname{CAT}(0)$ space $\left(Y_{1}, d_{Y_{1}}\right)$ and a map $g_{1}^{\prime}: V^{\prime} \rightarrow Y_{1}$ such that

$$
d_{Y_{1}}\left(g_{1}^{\prime}\left(x_{2}\right), g_{1}^{\prime}\left(x_{4}\right)\right) \geq d_{24}, \quad d_{Y_{1}}\left(g_{1}^{\prime}\left(x_{1}\right), g_{1}^{\prime}\left(x_{i}\right)\right) \leq d_{1 i}, \quad d_{Y_{1}}\left(g_{1}^{\prime}\left(x_{j}\right), g_{1}^{\prime}\left(x_{k}\right)\right)=d_{j k}
$$

for any $i, j, k \in\{2,3,4,5\}$ with $\{j, k\} \neq\{2,4\}$. Define a map $g_{1}: V \rightarrow Y_{1}$ by

$$
g_{1}\left(v_{i}\right)=g_{1}^{\prime}\left(x_{i}\right)
$$

for each $i \in\{1,2,3,4,5\}$. Then

$$
\begin{cases}d_{Y_{1}}\left(g_{1}\left(v_{i}\right), g_{1}\left(v_{j}\right)\right) \leq d_{i j}, & \text { if }\left\{v_{i}, v_{j}\right\} \in E, \\ d_{Y_{1}}\left(g_{1}\left(v_{i}\right), g_{1}\left(v_{j}\right)\right) \geq d_{i j}, & \text { if }\left\{v_{i}, v_{j}\right\} \notin E\end{cases}
$$


for any $i, j \in\{1,2,3,4,5\}$. Thus $g_{1}$ is a map from $V$ to a CAT(0) space with the desired properties.

CASE 2: $V_{1}^{\prime}$ admits an isometric embedding into $\mathbb{R}^{3}$, and $V_{2}^{\prime}$ does not, or vice versa. In this case, Lemma 9.5 implies that there exist a $\operatorname{CAT}(0)$ space $\left(Y_{2}, d_{Y_{2}}\right)$ and a map $g_{2}^{\prime}: V^{\prime} \rightarrow Y_{2}$ such that

$$
d_{Y_{2}}\left(g_{2}^{\prime}\left(x_{2}\right), g_{2}^{\prime}\left(x_{4}\right)\right) \geq d_{24}, \quad d_{Y_{2}}\left(g_{2}^{\prime}\left(x_{1}\right), g_{2}^{\prime}\left(x_{i}\right)\right) \leq d_{1 i}, \quad d_{Y_{2}}\left(g_{2}^{\prime}\left(x_{j}\right), g_{2}^{\prime}\left(x_{k}\right)\right)=d_{j k}
$$

for any $i, j, k \in\{2,3,4,5\}$ with $\{j, k\} \neq\{2,4\}$. Define $g_{2}: V \rightarrow Y_{2}$ by

$$
g_{2}\left(v_{i}\right)=g_{2}^{\prime}\left(x_{i}\right)
$$

for each $i \in\{1,2,3,4,5\}$. Then

$$
\begin{cases}d_{Y_{2}}\left(g_{2}\left(v_{i}\right), g_{2}\left(v_{j}\right)\right) \leq d_{i j}, & \text { if }\left\{v_{i}, v_{j}\right\} \in E, \\ d_{Y_{2}}\left(g_{2}\left(v_{i}\right), g_{2}\left(v_{j}\right)\right) \geq d_{i j}, & \text { if }\left\{v_{i}, v_{j}\right\} \notin E\end{cases}
$$

for any $i, j \in\{1,2,3,4,5\}$. Thus $g_{2}$ is a map from $V$ to a $\operatorname{CAT}(0)$ space with the desired properties.

CASE 3: Neither $V_{1}^{\prime}$ nor $V_{2}^{\prime}$ admits an isometric embedding into $\mathbb{R}^{3}$. We divide CASE 3 into four subcases.

SUBCASE 3A: $V_{1}^{\prime}$ is over-distance with respect to $\left\{x_{2}, x_{3}\right\}$ or $\left\{x_{2}, x_{5}\right\}$, and $V_{2}^{\prime}$ is over-distance with respect to $\left\{x_{4}, x_{3}\right\}$ or $\left\{x_{4}, x_{5}\right\}$. In this case, Lemma 9.3 implies that there exist a CAT(0) space $\left(Y_{3}, d_{Y_{3}}\right)$ and a map $g_{3}^{\prime}: V^{\prime} \rightarrow Y_{3}$ such that

$$
d_{Y_{3}}\left(g_{3}^{\prime}\left(x_{2}\right), g_{3}^{\prime}\left(x_{4}\right)\right) \geq d_{24}, \quad d_{Y_{3}}\left(g_{3}^{\prime}\left(x_{i}\right), g_{3}^{\prime}\left(x_{j}\right)\right)=d_{i j}
$$

for any $i, j \in\{1,2,3,4,5\}$ with $\{i, j\} \neq\{2,4\}$. Define $g_{3}: V \rightarrow Y_{3}$ by

$$
g_{3}\left(v_{i}\right)=g_{3}^{\prime}\left(x_{i}\right)
$$

for each $i \in\{1,2,3,4,5\}$. Then

$$
\begin{cases}d_{Y_{3}}\left(g_{3}\left(v_{i}\right), g_{3}\left(v_{j}\right)\right)=d_{i j}, & \text { if }\left\{v_{i}, v_{j}\right\} \in E, \\ d_{Y_{3}}\left(g_{3}\left(v_{i}\right), g_{3}\left(v_{j}\right)\right) \geq d_{i j}, & \text { if }\left\{v_{i}, v_{j}\right\} \notin E\end{cases}
$$

for any $i, j \in\{1,2,3,4,5\}$. Thus $g_{3}$ is a map from $V$ to a $\operatorname{CAT}(0)$ space with the desired properties.

SUBCASE 3B: $V_{1}^{\prime}$ is under-distance with respect to $\left\{x_{2}, x_{3}\right\}$ or $\left\{x_{2}, x_{5}\right\}$, and $V_{2}^{\prime}$ is under-distance with respect to $\left\{x_{4}, x_{3}\right\}$ or $\left\{x_{4}, x_{5}\right\}$. In this case, Lemma 9.4 implies that there exist a $\operatorname{CAT}(0)$ space $\left(Y_{4}, d_{Y_{4}}\right)$ and a map $g_{4}^{\prime}: V^{\prime} \rightarrow Y_{4}$ such that

$$
d_{Y_{4}}\left(g_{4}^{\prime}\left(x_{2}\right), g_{4}^{\prime}\left(x_{4}\right)\right) \geq d_{24}, \quad d_{Y_{4}}\left(g_{4}^{\prime}\left(x_{1}\right), g_{4}^{\prime}\left(x_{i}\right)\right) \leq d_{1 i}, \quad d_{Y_{4}}\left(g_{4}^{\prime}\left(x_{j}\right), g_{4}^{\prime}\left(x_{k}\right)\right)=d_{j k}
$$

for any $i, j, k \in\{2,3,4,5\}$ with $\{j, k\} \neq\{2,4\}$. Define $g_{4}: V \rightarrow Y_{4}$ by

$$
g_{4}\left(v_{i}\right)=g_{4}^{\prime}\left(x_{i}\right)
$$

for each $i \in\{1,2,3,4,5\}$. Then

$$
\begin{cases}d_{Y_{4}}\left(g_{4}\left(v_{i}\right), g_{4}\left(v_{j}\right)\right) \leq d_{i j}, & \text { if }\left\{v_{i}, v_{j}\right\} \in E, \\ d_{Y_{4}}\left(g_{4}\left(v_{i}\right), g_{4}\left(v_{j}\right)\right) \geq d_{i j}, & \text { if }\left\{v_{i}, v_{j}\right\} \notin E\end{cases}
$$

for any $i, j \in\{1,2,3,4,5\}$. Thus $g_{4}$ is a map from $V$ to a $\operatorname{CAT}(0)$ space with the desired properties.

SUBCASE 3C: $V_{1}^{\prime}$ is under-distance with respect to $\left\{x_{2}, x_{3}\right\}$ and $\left\{x_{2}, x_{5}\right\}$, and $V_{2}^{\prime}$ is over-distance with respect to $\left\{x_{4}, x_{3}\right\}$ and $\left\{x_{4}, x_{5}\right\}$. In this case, if neither $\left\{x_{3}, x_{1}, x_{2}, x_{4}\right\}$ nor $\left\{x_{5}, x_{1}, x_{2}, x_{4}\right\}$ admits an isometric embedding into $\mathbb{R}^{3}$, then Corollary 7.13 implies that $\left\{x_{3}, x_{1}, x_{2}, x_{4}\right\}$ is over-distance with respect to $\left\{x_{3}, x_{2}\right\}$ or $\left\{x_{3}, x_{4}\right\}$, and $\left\{x_{5}, x_{1}, x_{2}, x_{4}\right\}$ is over-distance with respect to $\left\{x_{5}, x_{2}\right\}$ or $\left\{x_{5}, x_{4}\right\}$, and therefore the existence of a map from $V$ to a CAT(0) space with the desired properties is proved in exactly the same way as in SUBCASE 3A. If $\left\{x_{3}, x_{1}, x_{2}, x_{4}\right\}$ or $\left\{x_{5}, x_{1}, x_{2}, x_{4}\right\}$ embeds isometrically into $\mathbb{R}^{3}$, then the existence of a map from $V$ to a CAT(0) space with the desired properties is proved in exactly the same way as in CASE 1 and CASE 2. 
SUBCASE 3D: $V_{1}^{\prime}$ is over-distance with respect to $\left\{x_{2}, x_{3}\right\}$ and $\left\{x_{2}, x_{5}\right\}$, and $V_{2}^{\prime}$ is under-distance with respect to $\left\{x_{4}, x_{3}\right\}$ and $\left\{x_{4}, x_{5}\right\}$. In this case, the existence of a map from $V$ to a CAT(0) space with the desired properties is proved in exactly the same way as in SUBCASE 3c.

By Proposition 7.2, the above four subcases exhaust all possibilities in CASE 3. CASE 1, CASE 2 and CASE 3 exhaust all possibilities.

We have proved that a metric space $X$ satisfies the $G(0)$ condition for every graph $G$ containing at most five vertices whenever $X$ satisfies the $\otimes$-inequalities, which implies Theorem 1.3 by Proposition 1.9.

Proof of Theorem 1.3. It follows from Propositions 6.1, 6.2, 6.3, 6.4, 6.6, 6.7, 6.8 8.5 and 9.6 that a metric space $X$ satisfies the $G(0)$ condition for every graph $G$ that contains at most five vertices whenever $X$ satisfies the $\bigotimes$-inequalities. Therefore, Proposition 1.9 implies that a metric space $X$ containing at most five points admits an isometric embedding into a CAT(0) space whenever $X$ satisfies the $\otimes$-inequalities. Conversely, if a metric space $X$ containing at most five points admits an isometric embedding into a CAT(0) space, then $X$ satisfies the $\otimes$-inequalities because every CAT(0) space satisfies the $\otimes$-inequalities.

Acknowledgement: This work is supported in part by JSPS KAKENHI Grant Number JP16K17602.

The author would like to thank Yu Kitabeppu, Takefumi Kondo, Masato Mimura, Shin Nayatani and Hiroshi Tamaru for helpful discussions and a number of valuable comments.

\section{References}

[1] S. Alexander, V. Kapovitch, and A. Petrunin. Alexandrov meets Kirszbraun. Proceedings of the Gökova Geometry-Topology Conference 2010. Int. Press, Somerville, MA, 2011, 88-109.

[2] A. Andoni, A. Naor, and O. Neiman. Snowflake universality of Wasserstein spaces. Ann. Sci. Éc. Norm. Supér. (4), 51(3):657-700, 2018.

[3] I. D. Berg and I. G. Nikolaev. Quasilinearization and curvature of Aleksandrov spaces. Geom. Dedicata, 133:195-218, 2008.

[4] M. R. Bridson and A. Haefliger. Metric spaces of non-positive curvature, volume 319 of Grundlehren der Mathematischen Wissenschaften [Fundamental Principles of Mathematical Sciences]. Springer-Verlag, Berlin, 1999.

[5] D. Burago, Y. Burago, and S. Ivanov. A course in metric geometry, volume 33 of Graduate Studies in Mathematics. American Mathematical Society, Providence, RI, 2001.

[6] Y. Burago, M. Gromov, and G. Perelman. A. D. Aleksandrov spaces with curvatures bounded below. Uspekhi Mat. Nauk, 47(2(284)):3-51, 222, 1992.

[7] P. Enflo. On the nonexistence of uniform homeomorphisms between $L_{p}$-spaces. Ark. Mat., 8:103-105, 1969.

[8] A. Eskenazis, M. Mendel, and A. Naor. Nonpositive curvature is not coarsely universal. Invent. Math., 217(3):833-886, 2019.

[9] M. Gromov. Metric structures for Riemannian and non-Riemannian spaces, volume 152 of Progress in Mathematics. Birkhäuser Boston, Inc., Boston, MA, 1999. Based on the 1981 French original [ MR0682063 (85e:53051)], With appendices by M. Katz, P. Pansu and S. Semmes, Translated from the French by Sean Michael Bates.

[10] M. Gromov. CAT( () -spaces: construction and concentration. Zap. Nauchn. Sem. S.-Peterburg. Otdel. Mat. Inst. Steklov. (POMI), 280(Geom. i Topol. 7):100-140, 299-300, 2001.

[11] B. Kleiner and B. Leeb. Rigidity of quasi-isometries for symmetric spaces and Euclidean buildings. Inst. Hautes Études Sci. Publ. Math., (86):115-197 (1998), 1997.

[12] T. Kondo, T. Toyoda, and T. Uehara. On a question of Gromov about the Wirtinger inequalities. Geom. Dedicata, 195(1):203-214, 2018.

[13] U. Lang and V. Schroeder. Kirszbraun's theorem and metric spaces of bounded curvature. Geom. Funct. Anal., 7(3):535$560,1997$.

[14] N. Lebedeva, A. Petrunin and V. Zolotov. Bipolar comparison. Geom. Funct. Anal., 29(1):258-282, 2019.

[15] J. G. Rešetnjak. Non-expansive maps in a space of curvature no greater than K. Sibirsk. Mat. Ž, 9:918-927, 1968.

[16] T. Sato. An alternative proof of Berg and Nikolaev's characterization of CAT(0)-spaces via quadrilateral inequality. Arch. Math. (Basel), 93(5):487-490, 2009. 
[17] K.-T. Sturm. Probability measures on metric spaces of nonpositive curvature. In Heat kernels and analysis on manifolds, graphs, and metric spaces (Paris, 2002), volume 338 of Contemp. Math., pages 357-390. Amer. Math. Soc., Providence, RI, 2003.

[18] T. Toyoda. A non-geodesic analogue of Reshetnyak's majorization theorem. Preprint available at https://arxiv.org/abs/ 1907.09067, 2019. 